Supporting Information

\title{
Design, Synthesis, and Evaluation of Carbamate-Substituted Analogues of (+)-Discodermolide
}

Amos B. Smith, III, ${ }^{1 *}$ B. Scott Freeze, ${ }^{1}$ Matthew J. LaMarche, ${ }^{1}$ Tomoyasu Hirose, ${ }^{1}$ Ignacio Brouard, ${ }^{1}$ Paul V. Rucker, ${ }^{1}$ Ming Xian, ${ }^{1}$ Kurt F. Sundermann, ${ }^{2}$ Simon J. Shaw, ${ }^{2}$ Mark A. Burlingame, ${ }^{2}$ Susan Band Horwitz ${ }^{3}$, and David C. Myles ${ }^{2 *}$

${ }^{I}$ Department of Chemistry, University of Pennsylvania, Philadelphia, PA 19104

${ }^{2}$ Kosan Biosciences Inc. 3832 Bay Center Place, Hayward, CA 94545

${ }^{3}$ Department of Molecular Pharmacology, Albert Einstein College of Medicine, Bronx, NY, 10461 


\section{Table of Contents.}

Cover page

Table of contents

Materials and methods

Experimental details for transformations described in the main text $\ldots \ldots \ldots \ldots$

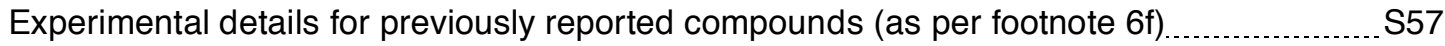

Materials and Methods. Reactions were carried out in oven or flame-dried glassware under an argon atmosphere, unless otherwise noted. All solvents were reagent grade. Diethyl ether $\left(\mathrm{Et}_{2} \mathrm{O}\right)$ and tetrahydrofuran (THF) were freshly distilled from sodium/benzophenone under argon. $n$ butyllithium and $t$-butyllithium were purchased from Aldrich. Reactions were magnetically stirred and monitored by thin layer chromotography (TLC) with $0.25 \mathrm{~mm}$ E. Merck pre-coated silica gel plates. Flash chromatography was performed with silica gel 60 (particle size $0.040-0.062 \mathrm{~mm}$ ) supplied by Silicycle and Sorbent Technologies. Yields refer to chromatographically and spectroscopically pure compounds, unless otherwise stated. Infrared spectra were recorded on a Jasco Model FT/IR-480 Plus spectrometer. Proton and carbon-13 NMR spectra were recorded on a Bruker AMX-500 spectrometer. Chemical shifts are reported relative to chloroform ( $\square 7.26)$, methanol ( $\square$ 3.31), acetonitrile ( $\square$ 1.94), or benzene $(\square 7.15)$ for ${ }^{1} \mathrm{H}-\mathrm{NMR}$ and either chloroform

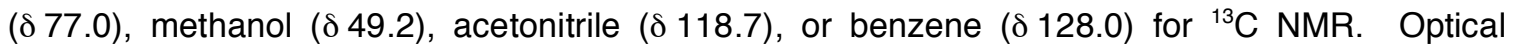
rotations were measured on a Perkin-Elmer model 241 polarimeter. High resolution mass spectra were measured at the University of Pennsylvania Mass Spectrometry Service Center. 


\section{Experimental Details}

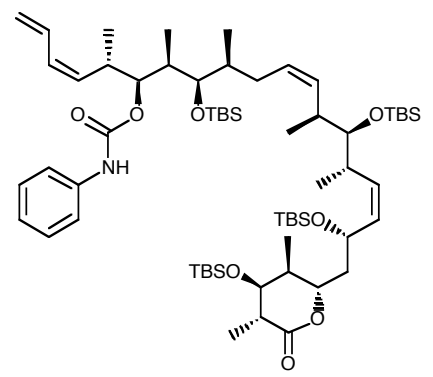

$(+)-S 1$

Phenyl carbamate (+)-S1: A solution of alcohol (+)-5 $(7.8 \mathrm{mg}, 7.86 \mu \mathrm{mol})$ in $\mathrm{CH}_{2} \mathrm{Cl}_{2}(1.73 \mathrm{~mL})$ was treated with phenyl isocyanate $(26 \mu \mathrm{L}, 0.236 \mathrm{mmol})$ in pyridine $(0.79 \mathrm{~mL})$ at room temperature for $16 \mathrm{hr}$. The reaction mixture was quenched with saturated $\mathrm{NH}_{4} \mathrm{Cl}(5 \mathrm{~mL})$, diluted with $\mathrm{CH}_{2} \mathrm{Cl}_{2}(5 \mathrm{~mL})$. The resulting layers were separated, and the aqueous layer was extracted with $\mathrm{CH}_{2} \mathrm{Cl}_{2}(3 \times 7 \mathrm{~mL})$. The combined organic layers were washed with saturated $\mathrm{NH}_{4} \mathrm{Cl}(1 \times 10$ $\mathrm{mL})$ and saturated brine $(1 \times 10 \mathrm{~mL})$, dried over $\mathrm{Na}_{2} \mathrm{SO}_{4}$, filtered, and concentrated. Flash chromatograpy (9\% ethyl acetate/hexanes) gave tetra-TBS carbamate (+)-S1 (7.6 mg, 87\%) as a colorless oil. $[\square]_{D}^{23}+49^{\circ}\left(c 0.35, \mathrm{CHCl}_{3}\right)$; IR (film, NaCl) 3342, 2957, 2857, 1734, 1252, 1047, $775 \mathrm{~cm}^{-1} ;{ }^{1} \mathrm{H}$ NMR $\left(500 \mathrm{MHz}, \mathrm{CDCl}_{3}\right) \square 7.34(\mathrm{~d}, J=7.7 \mathrm{~Hz}, 2 \mathrm{H}), 7.22($ app t, $J=7.9 \mathrm{~Hz}, 2 \mathrm{H})$, $7.03(\mathrm{~m}, 1 \mathrm{H}), 6.61$ (ddd, $J=17.0,10.6,10.6 \mathrm{~Hz}, 1 \mathrm{H}), 6.52(\mathrm{br} \mathrm{s}, 1 \mathrm{H}), 6.00$ (app t, $J=11.0 \mathrm{~Hz}$ 1H), $5.38(\operatorname{appt}, J=10.4 \mathrm{~Hz}, 1 \mathrm{H}), 5.30(\mathrm{~m}, 1 \mathrm{H}), 5.28(\mathrm{dd}, J=11.2,7.8 \mathrm{~Hz}, 1 \mathrm{H}), 5.24(\mathrm{~d}, J=10.6$ $\mathrm{Hz}, 1 \mathrm{H}), 5.19(\mathrm{~d}, J=15.9 \mathrm{~Hz}, 1 \mathrm{H}), 5.12(\mathrm{~m}, 2 \mathrm{H}), 4.80(\mathrm{~m}, 2 \mathrm{H}), 4.49$ (ddd, $J=10.8,10.8,1.3 \mathrm{~Hz}$, 1H), $3.61(\operatorname{app~t}, J=2.5 \mathrm{~Hz}, 1 \mathrm{H}), 3.49(\operatorname{app~t}, J=4.5 \mathrm{~Hz}, 1 \mathrm{H}), 3.28(\operatorname{app~t}, J=5.1 \mathrm{~Hz}, 1 \mathrm{H}), 3.03$ (app dq, $J=16.8,6.6 \mathrm{~Hz}, 1 \mathrm{H}), 2.60(\mathrm{~m}, 2 \mathrm{H}), 2.53(\mathrm{~m}, 1 \mathrm{H}), 1.92(\mathrm{~m}, 3 \mathrm{H}), 1.78$ (dddd, $J=17.1,6.6$, 6.6, $2.0 \mathrm{~Hz}, 1 \mathrm{H}), 1.72(\mathrm{~m}, 1 \mathrm{H}), 1.68(\mathrm{~m}, 1 \mathrm{H}), 1.57$ (ddd, $J=14.0,11.2,2.0 \mathrm{~Hz}, 1 \mathrm{H}), 1.21(\mathrm{~d}, J=$ $7.6 \mathrm{~Hz}, 3 \mathrm{H}), 1.01(\mathrm{~d}, J=6.8 \mathrm{~Hz}, 3 \mathrm{H}), 0.95(\mathrm{~d}, J=6.6 \mathrm{~Hz}, 6 \mathrm{H}), 0.94(\mathrm{~d}, J=6.4 \mathrm{~Hz}, 3 \mathrm{H}), 0.91$ (s, $9 \mathrm{H}), 0.88(\mathrm{~d}, J=6.7 \mathrm{~Hz}, 3 \mathrm{H}), 0.88(\mathrm{~s}, 9 \mathrm{H}), 0.86(\mathrm{~s}, 9 \mathrm{H}), 0.85(\mathrm{~s}, 9 \mathrm{H}), 0.80(\mathrm{~d}, J=6.7 \mathrm{~Hz}, 3 \mathrm{H})$, $0.081(\mathrm{~s}, 3 \mathrm{H}), 0.053(\mathrm{~s}, 3 \mathrm{H}), 0.046(\mathrm{~s}, 6 \mathrm{H}), 0.042(\mathrm{~s}, 3 \mathrm{H}), 0.028(\mathrm{~s}, 6 \mathrm{H}),-0.001(\mathrm{~s}, 3 \mathrm{H}) ;{ }^{13} \mathrm{C}$ NMR $\left(125 \mathrm{MHz}, \mathrm{CDCl}_{3}\right) \square$ 173.3, 158.6, 138.1, 135.3, 133.6, 133.5, 132.4, 132.1, 130.6, 130.0, 129.0, 
$127.0,123.3,118.1,80.0,79.1,77.0,76.3,74.9,64.6,44.2,42.8,38.2,37.9,37.5,35.1,34.5$, $34.1,31.8,26.3,26.2,25.9,25.7,18.5,18.4,18.1,17.9,17.6,17.2,16.4,16.0,14.4,14.1,10.3,-$ $3.1,-3.5,-3.6,-4.2,-4.3,-4.5,-4.9$; high resolution mass spectrum $\left(E^{+}\right) \mathrm{m} / \mathrm{z} 1134.7461$ $\left[(\mathrm{M}+\mathrm{Na})^{+}\right.$; calcd for $\left.\mathrm{C}_{62} \mathrm{H}_{113} \mathrm{NO}_{8} \mathrm{NaSi}_{4}: 1134.7441\right]$.

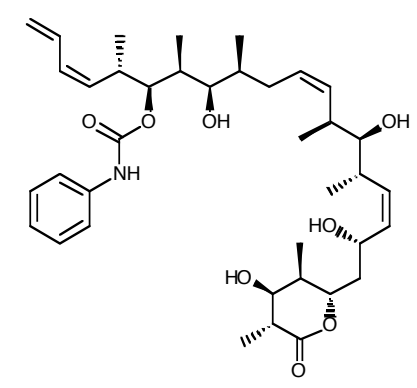

(+)-6

Phenyl carbamate tetra-ol (+)-6: Tetra-TBS carbamate (+)-S1 (7.0 mg, $6.30 \mu \mathrm{mol})$ was dissolved in $\mathrm{MeOH}(3.2 \mathrm{~mL})$. Aqueous $\mathrm{HCl}(6 \mathrm{~N}, 3.1 \mathrm{~mL})$ was added dropwise over $4 \mathrm{hr}$ at room temperature. The reaction mixture was stirred for $4 \mathrm{hr}$ further, diluted with water $(6 \mathrm{~mL})$ and EtOAc $(10 \mathrm{~mL})$. The resulting mixture was neutralized by solid $\mathrm{NaHCO}_{3}$, and separated. The aqueous layer was extracted with EtOAc $(3 \times 8 \mathrm{~mL})$. The combined organic layers were washed with saturated brine $(1 \times 15 \mathrm{~mL})$, dried over $\mathrm{Na}_{2} \mathrm{SO}_{4}$, filtered, and concentrated. Preparative TLC (9\% MeOH/CH $\left.\mathrm{Cl}_{2}\right)$ provided phenyl carbamate tetra-ol (+)-6 (3.4 mg, 83\%) as a colorless solid. $[\square]_{\mathrm{D}}^{23}+45$ (c $\left.0.17 \mathrm{MeOH}\right) ; \mathrm{IR}$ (film, NaCl) 3426, 2968, 1707, 1230, $753 \mathrm{~cm}^{-1} ;{ }^{1} \mathrm{H}$ NMR $(500 \mathrm{MHz}$, $\left.\mathrm{CD}_{3} \mathrm{CN}\right) \square 7.70(\mathrm{br} \mathrm{s}, 1 \mathrm{H}), 7.40(\mathrm{~d}, J=8.0 \mathrm{~Hz}, 2 \mathrm{H}), 7.28$ (app t, $\left.J=7.8 \mathrm{~Hz}, 2 \mathrm{H}\right), 7.02($ app t, $J=$ $7.4 \mathrm{~Hz}, 1 \mathrm{H}), 6.69$ (ddd, $J=16.8,10.6,10.6 \mathrm{~Hz}, 1 \mathrm{H}), 6.05(\operatorname{app~t}, J=11.0 \mathrm{~Hz}, 1 \mathrm{H}), 5.44(\mathrm{~m}, 2 \mathrm{H})$, $5.37(\mathrm{dd}, J=11.0,8.3 \mathrm{~Hz}, 1 \mathrm{H}), 5.21(\mathrm{~m}, 2 \mathrm{H}), 5.14(\mathrm{~d}, J=10.2 \mathrm{~Hz}, 1 \mathrm{H}), 4.82(\operatorname{app} t, J=6.0 \mathrm{~Hz}$, 1H), 4.51 (app t, $J=9.1 \mathrm{~Hz}, 1 \mathrm{H}), 4.44$ (ddd, $J=10.6,10.6,1.7 \mathrm{~Hz}, 1 \mathrm{H}), 3.60(\mathrm{~m}, 1 \mathrm{H}), 3.27(\mathrm{~m}$, 1H), $3.24(\mathrm{dd}, J=11.4,6.2 \mathrm{~Hz}, 1 \mathrm{H}), 3.11(\mathrm{~m}, 2 \mathrm{H}), 2.60(\mathrm{~m}, 2 \mathrm{H}), 2.55$ (dddd, $J=7.4,7.4,7.4,4.8$ $\mathrm{Hz}, 1 \mathrm{H}), 2.41(\mathrm{~m}, 1 \mathrm{H}), 1.84(\mathrm{~m}, 2 \mathrm{H}), 1.71(\mathrm{~m}, 2 \mathrm{H}), 1.64(\mathrm{~m}, 1 \mathrm{H}), 1.54$ (ddd, $J=13.8,10.6,2.5 \mathrm{~Hz}$, 1H), $1.18(\mathrm{~d}, J=7.3 \mathrm{~Hz}, 3 \mathrm{H}), 0.99(\mathrm{~d}, J=6.8 \mathrm{~Hz}, 6 \mathrm{H}), 0.96(\mathrm{~d}, J=6.9 \mathrm{~Hz}, 3 \mathrm{H}), 0.91(\mathrm{~d}, J=6.9$ $\mathrm{Hz}, 3 \mathrm{H}), 0.89(\mathrm{~d}, J=6.8 \mathrm{~Hz}, 3 \mathrm{H}), 0.84(\mathrm{~d}, J=6.5 \mathrm{~Hz}, 3 \mathrm{H}) ;{ }^{13} \mathrm{C} \mathrm{NMR}\left(125 \mathrm{MHz}, \mathrm{CD}_{3} \mathrm{CN}\right) \square 174.7$, 
155.0, 140.0, 135.0, 134.5, 134.0, 133.6, 133.4, 130.6, $129.8(2), 128.3,123.7,119.4,79.5,79.3$ $78.0,75.2,73.1,63.8,43.9,42.3,38.4,36.7,36.6,36.3,36.2,35.0,32.2,19.1,18.1,16.6,15.7$, 15.4, 13.0, 9.6; high resolution mass spectrum $\left(\mathrm{ES}^{+}\right) \mathrm{m} / \mathrm{z} 678.3976\left[(\mathrm{M}+\mathrm{Na})^{+}\right.$; calcd for $\left.\mathrm{C}_{38} \mathrm{H}_{57} \mathrm{NO}_{8} \mathrm{Na}: 678.3982\right]$.

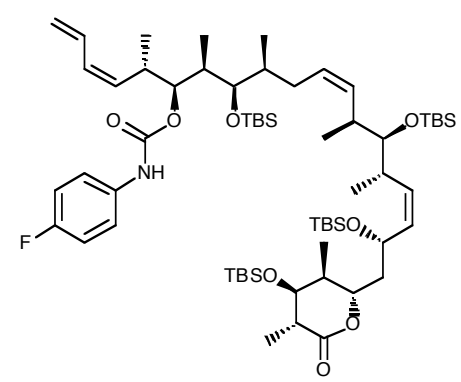

(+)-S2

p-Fluorophenyl carbamate (+)-S2: A solution of alcohol (+)-5 (10.7 mg, $10.8 \mu \mathrm{mol})$ in pyridine $(1.1 \mathrm{~mL})$ was treated with 4-fluorophenyl isocyanate $(44.4 \mathrm{mg}, 0.324 \mathrm{mmol})$ at room temperature for $3 \mathrm{~h} 20$ min. The reaction mixture was quenched with saturated $\mathrm{NH}_{4} \mathrm{Cl}(3 \mathrm{~mL})$, and diluted with $\mathrm{CH}_{2} \mathrm{Cl}_{2}(6 \mathrm{~mL})$. The resulting layers were separated, and the aqueous layer was extracted with $\mathrm{CH}_{2} \mathrm{Cl}_{2}(3 \times 6 \mathrm{~mL})$. The combined organic layers were washed with saturated brine $(1 \times 10 \mathrm{~mL})$, dried over $\mathrm{Na}_{2} \mathrm{SO}_{4}$, filtered, and concentrated. Preparative TLC (9\% ethyl acetate/hexanes) provided $10.2 \mathrm{mg}$ tetra-TBS carbamate $(+)$-S2 $(84 \%)$ as a colorless oil. $[\square]_{\mathrm{D}}^{23}+42^{\circ}(c \cdot 0.2$, $\mathrm{CHCl}_{3}$ ); IR (film, NaCl) 3336, 2957, 2857, 1734, 1513, 1213, 1046, $835 \mathrm{~cm}^{-1} ;{ }^{1} \mathrm{H} \mathrm{NMR}(500 \mathrm{MHz}$, $\left.\mathrm{CDCl}_{3}\right) \square 7.28$ (br s, 2H), $6.96($ app t, $J=8.6 \mathrm{~Hz}, 1 \mathrm{H}), 6.60$ (ddd, $\left.J=16.9,10.6,10.6 \mathrm{~Hz}, 1 \mathrm{H}\right)$, 6.51 (br s, 1H), $6.00($ app t, $J=11.0 \mathrm{~Hz}, 1 \mathrm{H}), 5.37($ app t, $J=10.5 \mathrm{~Hz}, 1 \mathrm{H}), 5.25(\mathrm{~m}, 5 \mathrm{H}), 5.13$ (dd, $J=16.8,8.2 \mathrm{~Hz}, 1 \mathrm{H}), 4.78(\mathrm{~m}, 2 \mathrm{H}), 4.49(\operatorname{app~t}, J=10.6 \mathrm{~Hz}, 1 \mathrm{H}), 3.61(\operatorname{app~t}, J=2.3 \mathrm{~Hz}$, 1H), $3.48(\operatorname{appt}, J=4.4 \mathrm{~Hz}, 1 \mathrm{H}), 3.28(\operatorname{app~t}, J=5.1 \mathrm{~Hz}, 1 \mathrm{H}), 3.02(\operatorname{app} \mathrm{dq}, J=16.8,6.7 \mathrm{~Hz}, 1 \mathrm{H})$, $2.61(\mathrm{~m}, 2 \mathrm{H}), 2.53(\operatorname{app~dq}, J=13.4,6.8 \mathrm{~Hz}, 1 \mathrm{H}), 1.92(\mathrm{~m}, 3 \mathrm{H}), 1.78$ (dddd, $J=15.8,6.6,6.6,2.1$ $\mathrm{Hz}, 1 \mathrm{H}), 1.69(\mathrm{~m}, 2 \mathrm{H}), 1.57(\mathrm{ddd}, J=13.3,11.6,1.7 \mathrm{~Hz}, 1 \mathrm{H}), 1.21(\mathrm{~d}, J=7.6 \mathrm{~Hz}, 3 \mathrm{H}), 1.01(\mathrm{~d}, J=$ $6.7 \mathrm{~Hz}, 3 \mathrm{H}), 0.94(\mathrm{~d}, J=6.7 \mathrm{~Hz}, 3 \mathrm{H}), 0.94(\mathrm{~d}, J=6.4 \mathrm{~Hz}, 6 \mathrm{H}), 0.91(\mathrm{~s}, 9 \mathrm{H}), 0.88(\mathrm{~s}, 9 \mathrm{H}), 0.88(\mathrm{~d}, J$ $=6.5 \mathrm{~Hz}, 3 \mathrm{H}), 0.86(\mathrm{~s}, 9 \mathrm{H}), 0.85(\mathrm{~s}, 9 \mathrm{H}), 0.80(\mathrm{~d}, J=6.8 \mathrm{~Hz}, 3 \mathrm{H}), 0.076(\mathrm{~s}, 3 \mathrm{H}), 0.052(\mathrm{~s}, 3 \mathrm{H})$, 
$0.047(\mathrm{~s}, 6 \mathrm{H}), 0.040(\mathrm{~s}, 3 \mathrm{H}), 0.031(\mathrm{~s}, 3 \mathrm{H}), 0.026(\mathrm{~s}, 3 \mathrm{H}),-0.004(\mathrm{~s}, 3 \mathrm{H}) ;{ }^{13} \mathrm{C} \mathrm{NMR}(125 \mathrm{MHz}$, $\left.\mathrm{CDCl}_{3}\right) \square 173.3,153.8,135.4,134.1,133.6,133.5,132.4,132.1,130.0,127.0,120.2,118.1$, $115.7,115.5,80.0,79.3,77.1,76.2,74.9,64.6,44.2,42.9,38.2,37.9,37.5,35.1,34.6,34.2$, $31.7,26.3,26.2,25.9,25.7,19.0,18.9,18.5,18.4,17.6,17.2,16.4,15.9,14.5,14.1,10.3,-3.1$, 3.5, -3.6, -4.2, -4.3, -4.5, -4.9; high resolution mass spectrum $\left(\mathrm{ES}^{+}\right) \mathrm{m} / \mathrm{z} 1152.7358\left[(\mathrm{M}+\mathrm{Na})^{+}\right.$; calcd for $\left.\mathrm{C}_{62} \mathrm{H}_{112} \mathrm{NO}_{8} \mathrm{FNaSi}_{4}: 1152.7347\right]$.

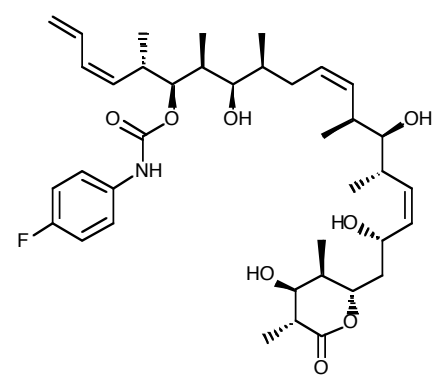

$(+)-7$

p-Fluorophenyl carbamate tetra-ol (+)-7: Tetra-TBS carbamate (+)-S2 (10.0 mg, $0.0089 \mathrm{mmol})$ was dissolved in $\mathrm{MeOH}(1.0 \mathrm{~mL})$ and stirred for $15 \mathrm{~min}$ at room temperature. Aqueous hydrochloric acid (3N, $5.0 \mathrm{~mL})$ was added in $200-400 \mu \mathrm{L}$ portions over 4 hours at a rate which minimized precipitation (ca. 10 to 15 min intervals). An additional $2 \mathrm{~mL}$ of $3 \mathrm{~N}$ aq $\mathrm{HCl}$ was added over $1 \mathrm{~h}$ at $15 \mathrm{~min}$ intervals, and the sides of the flask/stir bar were rinsed with $2.0 \mathrm{~mL}$ of $\mathrm{MeOH}$. After $12 \mathrm{~h}$ the solution was quenched with $\mathrm{NaHCO}_{3}(\mathrm{~s})$, diluted with $30 \mathrm{~mL}$ of water and extracted $3 \times$ with EtOAc. The combined organic extracts were dried $\left(\mathrm{Na}_{2} \mathrm{SO}_{4}\right)$, filtered, and concentrated. Preparative TLC (80\% EtOAc/ $\left.\mathrm{CH}_{2} \mathrm{Cl}_{2}\right)$ gave $p$-fluorophenyl carbamate tetra-ol (+)-7 (3.0 mg, 50\% yield). $[\square]_{D}^{23}+131$ (c $\left.0.075 \mathrm{MeOH}\right)$; IR (film, NaCl) 3429, 2970, 1708, 1512, $1215 \mathrm{~cm}^{-1} ;{ }^{1} \mathrm{H}$ NMR (500 MHz, CD $\left.{ }_{3} \mathrm{CN}\right) \square 7.71(\mathrm{br} \mathrm{s}, 1 \mathrm{H}), 7.39(\mathrm{dd}, J=8.4,4.8 \mathrm{~Hz}, 2 \mathrm{H}), 7.03(\operatorname{app~t}, J=8.9 \mathrm{~Hz}, 2 \mathrm{H})$, 6.69 (ddd, $J=16.9,10.7,10.7 \mathrm{~Hz}, 1 \mathrm{H}), 6.04(\operatorname{app~t}, J=11.0 \mathrm{~Hz}, 1 \mathrm{H}), 5.44(\mathrm{~m}, 2 \mathrm{H}), 5.37$ (dd, $J=$ 11.1, 8.2 Hz, 1H), $5.23(\mathrm{~m}, 2 \mathrm{H}), 5.20(\mathrm{dd}, J=6.4,4.5 \mathrm{~Hz}, 1 \mathrm{H}), 5.13(\mathrm{~d}, J=10.2 \mathrm{~Hz}, 1 \mathrm{H}), 4.82$ (app t, $J=6.0 \mathrm{~Hz}, 1 \mathrm{H}), 4.50(\mathrm{~m}, 1 \mathrm{H}), 4.44(\mathrm{ddd}, J=10.3,10.3,2.0 \mathrm{~Hz}, 1 \mathrm{H}), 3.60(\mathrm{~m}, 1 \mathrm{H}), 3.26(\mathrm{~d}$, $J=4.6 \mathrm{~Hz}, 1 \mathrm{H}), 3.23(\mathrm{~m}, 1 \mathrm{H}), 2.81(\mathrm{~d}, J=5.1 \mathrm{~Hz}, 1 \mathrm{H}), 2.63(\mathrm{~d}, J=5.3 \mathrm{~Hz}, 1 \mathrm{H}), 2.60(\mathrm{~m}, 2 \mathrm{H})$, 
$2.58(\mathrm{~d}, J=6.7 \mathrm{~Hz}, 1 \mathrm{H}), 2.55(\mathrm{ddd}, J=14.7,7.4,4.7 \mathrm{~Hz}, 1 \mathrm{H}), 2.42(\mathrm{~m}, 1 \mathrm{H}), 1.71(\mathrm{ddd}, J=14.3$, 10.2, $2.0 \mathrm{~Hz}, 1 \mathrm{H}), 1.63(\mathrm{~m}, 1 \mathrm{H}), 1.54$ (ddd, $J=14.2,10.6,2.5 \mathrm{~Hz}, 1 \mathrm{H}), 1.18(\mathrm{~d}, J=7.3 \mathrm{~Hz}, 3 \mathrm{H})$, $0.99(\mathrm{~d}, J=6.9 \mathrm{~Hz}, 3 \mathrm{H}), 0.98(\mathrm{~d}, J=6.8 \mathrm{~Hz}, 3 \mathrm{H}), 0.96(\mathrm{~d}, J=6.9 \mathrm{~Hz}, 3 \mathrm{H}), 0.91(\mathrm{~d}, J=6.9 \mathrm{~Hz}$, $3 \mathrm{H}), 0.89(\mathrm{~d}, J=6.8 \mathrm{~Hz}, 3 \mathrm{H}), 0.84(\mathrm{~d}, J=6.6 \mathrm{~Hz}, 3 \mathrm{H}) ;{ }^{13} \mathrm{C} \mathrm{NMR}\left(125 \mathrm{MHz}, \mathrm{CD}_{3} \mathrm{CN}\right) \square$ 174.7, $155.1,136.2,135.0,134.5,134.0,133.6,133.4,130.6,128.3,121.2,118.4,116.3,116.1,79.6$ $79.3,78.0,75.2,73.1,63.8,44.0,42.3,38.3,36.7,36.6,36.3,36.2,35.0,32.2,19.1,18.1,16.6$, 15.7, 15.3, 13.0, 9.6; high resolution mass spectrum $\left(\mathrm{ES}^{+}\right) \mathrm{m} / \mathrm{z} 696.3872\left[(\mathrm{M}+\mathrm{Na})^{+}\right.$; calcd for $\mathrm{C}_{38} \mathrm{H}_{56} \mathrm{NO}_{8} \mathrm{FNa:}$ 696.3888].

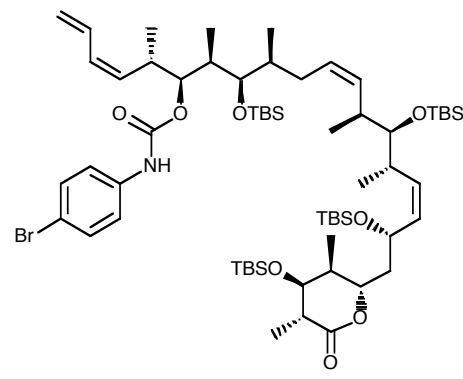

(+)-S3

p-Bromophenyl carbamate: A solution of alcohol (+)-5 $(6.2 \mathrm{mg}, 6.25 \mathrm{mmol})$ in DMF $(0.31 \mathrm{ml})$ was treated with 4-bromophenyl isocyanate $(37 \mathrm{~mL}, 0.188 \mathrm{mmol})$ at room temperature for $1 \mathrm{hr} 45$ min. The reaction mixture was quenched with saturated $\mathrm{NH}_{4} \mathrm{Cl}(2.0 \mathrm{~mL})$, and diluted with $\mathrm{CH}_{2} \mathrm{Cl}_{2}$ $(4 \mathrm{ml})$. The resulting layers were separated and the aqueous layer was extracted with $\mathrm{CH}_{2} \mathrm{Cl}_{2}(3$ x $5 \mathrm{~mL})$. The combined organic layers were washed with saturated brine $(1 \times 10 \mathrm{~mL})$, dried over $\mathrm{Na}_{2} \mathrm{SO}_{4}$, filtered, and concentrated. Preparative TLC (9\% ethyl acetate/hexanes) afforded $7.0 \mathrm{mg}$ tetra-TBS carbamate $(+)-\mathrm{S} 3(95 \%)$ as colorless oil. $[\square]_{\mathrm{D}}^{23}+42\left(c \quad 0.16, \mathrm{CHCl}_{3}\right) ; \mathrm{IR}($ film, $\mathrm{NaCl})$ $3335,2929,2857,1717,1219,1046,836 \mathrm{~cm}^{-1} ;{ }^{1} \mathrm{H}$ NMR $\left(500 \mathrm{MHz}, \mathrm{CHCl}_{3}\right) \square 7.37(\mathrm{~d}, J=8.8$ $\mathrm{Hz}, 2 \mathrm{H}), 7.24(\mathrm{~d}, J=8.0 \mathrm{~Hz}, 2 \mathrm{H}), 6.59$ (ddd, $J=16.9,10.6,10.6 \mathrm{~Hz}, 1 \mathrm{H}), 6.57$ (br s, 1H), 5.99 (app t, $J=11.0 \mathrm{~Hz}, 1 \mathrm{H}$ ), 5.33 (ddd, $J=21.0,10.4,10.4 \mathrm{~Hz}, 1 \mathrm{H}$ ), $5.28(\mathrm{dd}, J=11.2,7.7 \mathrm{~Hz}, 1 \mathrm{H}$ ), $5.23(\mathrm{~d}, J=10.6 \mathrm{~Hz}, 1 \mathrm{H}), 5.15(\mathrm{~m}, 2 \mathrm{H}), 4.78(\mathrm{~m}, 2 \mathrm{H}), 4.49$ (ddd, $J=10.4,10.4,1.3 \mathrm{~Hz}, 1 \mathrm{H}), 3.61$ 
(app t, $J=2.4 \mathrm{~Hz}, 1 \mathrm{H}), 3.48(\operatorname{app~t}, J=4.4 \mathrm{~Hz}, 1 \mathrm{H}), 3.27$ (dd, $J=5.5,4.7 \mathrm{~Hz}, 1 \mathrm{H}), 3.02$ (app dq, $J$ $=16.8,6.7 \mathrm{~Hz}, 1 \mathrm{H}), 2.60(\mathrm{~m}, 2 \mathrm{H}), 2.52(\mathrm{~m}, 1 \mathrm{H}), 1.92(\mathrm{~m}, 3 \mathrm{H}), 1.78(\mathrm{~m}, 1 \mathrm{H}), 1.72(\mathrm{~m}, 1 \mathrm{H}), 1.66(\mathrm{~m}$, 1H), 1.57 (ddd, $J=13.5,11.3,2.0,1 \mathrm{H}), 1.55(\mathrm{~m}, 2 \mathrm{H}), 1.21(\mathrm{~d}, J=7.6 \mathrm{~Hz}, 3 \mathrm{H}), 1.00(\mathrm{~d}, J=6.8 \mathrm{~Hz}$, 3H), $0.94(\mathrm{~d}, J=6.7 \mathrm{~Hz}, 3 \mathrm{H}), 0.94(\mathrm{~d}, J=7.0 \mathrm{~Hz}, 3 \mathrm{H}), 0.94$ (d, $J=6.6 \mathrm{~Hz}, 3 \mathrm{H}), 0.91(\mathrm{~s}, 9 \mathrm{H}), 0.88$ (d, $J=6.7 \mathrm{~Hz}, 3 \mathrm{H}), 0.88(\mathrm{~s}, 9 \mathrm{H}), 0.86(\mathrm{~s}, 9 \mathrm{H}), 0.85(\mathrm{~s}, 9 \mathrm{H}), 0.80(\mathrm{~d}, J=6.7 \mathrm{~Hz}, 3 \mathrm{H}), 0.07(\mathrm{~s}, 3 \mathrm{H})$,

0.05 (s, 3H), 0.05 (s, 3H), $0.04(\mathrm{~s}, 3 \mathrm{H}), 0.04(\mathrm{~s}, 3 \mathrm{H}), 0.03(\mathrm{~s}, 3 \mathrm{H}), 0.03(\mathrm{~s}, 3 \mathrm{H}),-0.006(\mathrm{~s}, 3 \mathrm{H}) ;{ }^{13} \mathrm{C}$ NMR $\left(125 \mathrm{MHz}, \mathrm{CHCl}_{3}\right) \square 173.3,153.5,137.3,135.4,133.6,133.3,132.4,132.1,131.9$ (2), $130.0,126.9,120.2,118.1,80.0,79.4,77.1,76.2,74.9,64.6,44.2,42.8,38.2,37.9,37.5,35.1$, $34.5,34.2,31.7,26.3,26.2,25.9,25.7,18.5,18.4,18.1,17.9,17.6,17.3,16.4,15.9,14.5,14.1$, $10.3,-3.1,-3.5,-3.6,-4.2,-4.3,-4.5,-4.9$; high resolution mass spectrum $\left(\mathrm{ES}^{+}\right) \mathrm{m} / \mathrm{z} 1212.6426$ $\left[(\mathrm{M}+\mathrm{Na})^{+}\right.$; calcd for $\left.\mathrm{C}_{62} \mathrm{H}_{112} \mathrm{NO}_{8} \mathrm{NaSi}_{4} \mathrm{Br}: 1212.6546\right]$.

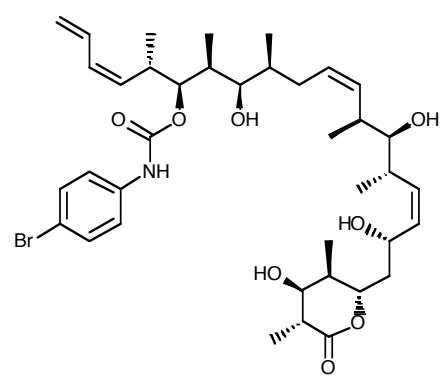

$(+)-8$

4-Bromophenyl carbamoyl tetra-ol (+)-8. Tetra-TBS carbamate (+)-S3 (6.0 mg, 0.005 mmol) was dissolved in $\mathrm{MeOH}(2.0 \mathrm{~mL})$ and stirred for $15 \mathrm{~min}$ at room temperature. Aqueous hydrochloric acid $(3 \mathrm{~N}, 5.0 \mathrm{~mL})$ was added in $200-400 \mu \mathrm{L}$ portions over 4 hours at a rate which minimized precipitation (ca. 10 to 15 min intervals). An additional $2 \mathrm{~mL}$ of $3 \mathrm{~N}$ aq $\mathrm{HCl}$ was added over $1 \mathrm{~h}$ at $15 \mathrm{~min}$ intervals, and the sides of the flask/stir bar were rinsed with $2.0 \mathrm{~mL}$ of $\mathrm{MeOH}$. After $12 \mathrm{~h}$ the solution was quenched with $\mathrm{NaHCO}_{3}(\mathrm{~s})$, diluted with $30 \mathrm{~mL}$ of water and extracted $3 \times$ with EtOAc. The combined organic extracts were dried $\left(\mathrm{Na}_{2} \mathrm{SO}_{4}\right)$, filtered, and concentrated.

Preparative TLC (80\% ethyl acetate $\left./ \mathrm{CH}_{2} \mathrm{Cl}_{2}\right)$ afforded $(+)-8(2.9 \mathrm{mg}, 80 \%$ yield $) . \quad[\square]_{D}^{23}+36(c$ 
0.06, MeOH); IR (film, NaCl) 3584, 2868, 1704, $1223 \mathrm{~cm}^{-1} ;{ }^{1} \mathrm{H}$ NMR (500 MHz, CD $\left.{ }_{3} \mathrm{CN}\right) \square 7.86$ (br s, $1 \mathrm{H}), 7.41$ (d, $J=8.9 \mathrm{~Hz}, 2 \mathrm{H}), 7.35$ (d, $J=8.6 \mathrm{~Hz}, 2 \mathrm{H}), 6.69$ (ddd, $J=16.9,10.5,10.5 \mathrm{~Hz}$, 1H), $6.04($ app t, $J=11.0 \mathrm{~Hz}, 1 \mathrm{H}), 6.04(\operatorname{app~t}, J=11.0 \mathrm{~Hz}, 1 \mathrm{H}), 5.43(\mathrm{~m}, 2 \mathrm{H}), 5.36(\mathrm{dd}, J=11.1$, 8.3 Hz, 1H), $5.21(\mathrm{~m}, 3 \mathrm{H}), 5.13(\mathrm{~d}, J=10.3 \mathrm{~Hz}, 1 \mathrm{H}), 4.82(\mathrm{app} t, J=6.0 \mathrm{~Hz}, 1 \mathrm{H}), 4.50(\mathrm{~m}, 1 \mathrm{H})$, $4.44(\mathrm{dd}, J=10.3,2.1 \mathrm{~Hz}, 1 \mathrm{H}), 3.60(\operatorname{app~t}, J=4.2 \mathrm{~Hz}, 1 \mathrm{H}), 3.23(\mathrm{~m}, 1 \mathrm{H}), 3.09(\mathrm{~m}, 2 \mathrm{H}), 2.61(\mathrm{~m}$, 2H), $2.55(a p p d q, J=4.7,7.4 \mathrm{~Hz}, 1 \mathrm{H}), 2.41(\mathrm{~m}, 1 \mathrm{H}), 1.83(\mathrm{~m}, 2 \mathrm{H}), 1.73(\mathrm{~m}, 2 \mathrm{H}), 1.63(\mathrm{~m}, 1 \mathrm{H})$, $1.53(\mathrm{ddd}, J=14.4,10.5,2.5 \mathrm{~Hz}, 1 \mathrm{H}), 1.18(\mathrm{~d}, J=7.4 \mathrm{~Hz}, 3 \mathrm{H}), 0.98(\mathrm{~d}, J=6.9 \mathrm{~Hz}, 3 \mathrm{H}), 0.98(\mathrm{~d}, J$ $=6.8 \mathrm{~Hz}, 3 \mathrm{H}), 0.96(\mathrm{~d}, J=6.9 \mathrm{~Hz}, 3 \mathrm{H}), 0.91(\mathrm{~d}, J=6.8 \mathrm{~Hz}, 3 \mathrm{H}), 0.89(\mathrm{~d}, J=6.7 \mathrm{~Hz}, 3 \mathrm{H}), 0.84(\mathrm{~d}$, $J=6.6 \mathrm{~Hz}, 3 \mathrm{H}) ;{ }^{13} \mathrm{C}$ NMR $\left(125 \mathrm{MHz}, \mathrm{CD}_{3} \mathrm{CN}\right) \square 175.0,154.8,135.0,134.4,134.0,133.6,133.4$, $132.6,130.6,128.3,121.1,118.3,116.3,116.1,79.8,79.3,78.0,75.2,73.1,63.8,43.9,42.3$, $38.3,36.7,36.6,36.3,35.0,32.2,30.6,19.1,18.1,16.6,15.7,15.3,13.1,9.6$; high resolution mass spectrum $\left(\mathrm{ES}^{+}\right) \mathrm{m} / \mathrm{z} 756.3075\left[(\mathrm{M}+\mathrm{Na})^{+}\right.$; calcd for $\mathrm{C}_{38} \mathrm{H}_{56} \mathrm{NO}_{8} \mathrm{NaBr}$ : 756.3087].

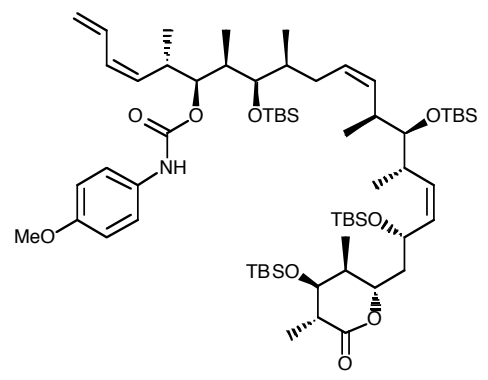

(+)-S4

p-Methoxyphenyl carbamate (+)-S4: A solution of alcohol (+)-5 (8.2 $\mathrm{mg}, 8.27 \mathrm{mmol})$ in pyridine (0.83 $\mathrm{mL})$ was treated with 4-methoxyphenyl isocyanate $(32 \mu \mathrm{L}, 0.248 \mathrm{mmol})$ at room temperature for $22 \mathrm{hr}$. The reaction mixture was quenched with saturated $\mathrm{NH}_{4} \mathrm{Cl}(2 \mathrm{~mL})$, and diluted with $\mathrm{CH}_{2} \mathrm{Cl}_{2}(5 \mathrm{~mL})$. The resulting layers were separated, and the aqueous layer was extracted with $\mathrm{CH}_{2} \mathrm{Cl}_{2}(3 \times 5 \mathrm{~mL})$. The combined organic layers were washed with saturated brine ( $1 \times 8 \mathrm{~mL}$ ), dried over $\mathrm{Na}_{2} \mathrm{SO}_{4}$, filtered, and concentrated. Preparative TLC (9\% ethyl acetate/ hexanes) provided $8.7 \mathrm{mg}$ tetra-TBS carbamate $(+)-\mathrm{S} 4(93 \%)$ as a colorless oil. $[\square]_{\mathrm{D}}^{23}+58^{\circ}(c 0.2$, 
$\mathrm{CHCl}_{3}$ ); IR (film, NaCl) 3347, 2929, 1733, 1516, 1046, $775 \mathrm{~cm}^{-1} ;{ }^{1} \mathrm{H}$ NMR (500 MHz, $\left.\mathrm{CHCl}_{3}\right)$ $7.23(\mathrm{~m}, 2 \mathrm{H}), 6.82(\mathrm{~d}, J=8.9 \mathrm{~Hz}, 2 \mathrm{H}), 6.60(\mathrm{ddd}, J=8.3,8.3,6.3 \mathrm{~Hz}, 1 \mathrm{H}), 6.39(\mathrm{br} \mathrm{s}, 1 \mathrm{H}), 6.00$ (app t, $J=11.0 \mathrm{~Hz}, 1 \mathrm{H}), 5.37(\operatorname{app~t}, J=10.4 \mathrm{~Hz}, 1 \mathrm{H}), 5.30(\mathrm{~m}, 2 \mathrm{H}), 5.28(\mathrm{dd}, J=11.2,8.0 \mathrm{~Hz}$, $1 \mathrm{H}), 5.19(\mathrm{~d}, J=16.3 \mathrm{~Hz}, 1 \mathrm{H}), 5.12(\mathrm{~m}, 2 \mathrm{H}), 4.78(\mathrm{~m}, 2 \mathrm{H}), 4.49(\operatorname{app~t}, J=10.4 \mathrm{~Hz}, 1 \mathrm{H}), 3.76(\mathrm{~s}$, 3H), $3.61(\operatorname{app~t}, J=2.2 \mathrm{~Hz}, 1 \mathrm{H}), 3.49(\operatorname{app~t}, J=4.0 \mathrm{~Hz}, 1 \mathrm{H}), 3.27(\operatorname{app~t}, J=5.1 \mathrm{~Hz}, 1 \mathrm{H}), 3.01$ (app dq, $J=16.6,6.7 \mathrm{~Hz}, 1 \mathrm{H}), 2.61(\mathrm{~m}, 2 \mathrm{H}), 2.53(\mathrm{~m}, 1 \mathrm{H}), 1.90(\mathrm{~m}, 3 \mathrm{H}), 1.78(\mathrm{~m}, 1 \mathrm{H}), 1.70(\mathrm{~m}$, 2H), 1.57 (ddd, $J=13.4,11.8,1.6 \mathrm{~Hz}, 1 \mathrm{H}), 1.20(\mathrm{~d}, J=7.6 \mathrm{~Hz}, 3 \mathrm{H}), 1.00(\mathrm{~d}, J=6.7 \mathrm{~Hz}, 3 \mathrm{H}$ ), 0.95 (d, $J=5.4 \mathrm{~Hz}, 3 \mathrm{H}), 0.94(\mathrm{~d}, J=6.5 \mathrm{~Hz}, 6 \mathrm{H}), 0.91(\mathrm{~s}, 9 \mathrm{H}), 0.88(\mathrm{~s}, 9 \mathrm{H}), 0.88(\mathrm{~d}, J=5.7 \mathrm{~Hz}, 3 \mathrm{H})$, $0.86(\mathrm{~s}, 9 \mathrm{H}), 0.85(\mathrm{~s}, 9 \mathrm{H}), 0.79(\mathrm{~d}, J=5.7 \mathrm{~Hz}, 3 \mathrm{H}) 0.078(\mathrm{~s}, 3 \mathrm{H}), 0.052(\mathrm{~s}, 3 \mathrm{H}), 0.044(\mathrm{~s}, 9 \mathrm{H})$, $0.028(\mathrm{~s}, 6 \mathrm{H}),-0.003(\mathrm{~s}, 3 \mathrm{H}) ;{ }^{13} \mathrm{C}$ NMR $\left(125 \mathrm{MHz}, \mathrm{CHCl}_{3}\right) \square$ 173.3, 153.5, 135.3, 133.6, 132.5, $132.2,131.0,129.9,127.1,120.7,118.0,114.2$, (two quaternary aromatic signals were significantly broadened and could not be resolved), 80.0, 79.0, 77.0, 76.2, 74.9, 64.6, 55.5, 44.2, $42.9,38.2,38.0,37.5,35.0,34.6,34.2,31.8,26.3,26.2,25.9,25.7,18.5,18.4,18.1,18.0,17.6$, $17.2,16.4,16.0,14.4,14.1,10.3,-3.1,-3.5,-3.6,-4.2,-4.3,-4.5,-4.9$; high resolution mass spectrum $\left(\mathrm{ES}^{+}\right) \mathrm{m} / \mathrm{z} 1164.7537$ [(M+Na) ${ }^{+}$; calcd for $\mathrm{C}_{63} \mathrm{H}_{115} \mathrm{NO}_{9} \mathrm{NaSi}_{4}:$ 1164.7547].

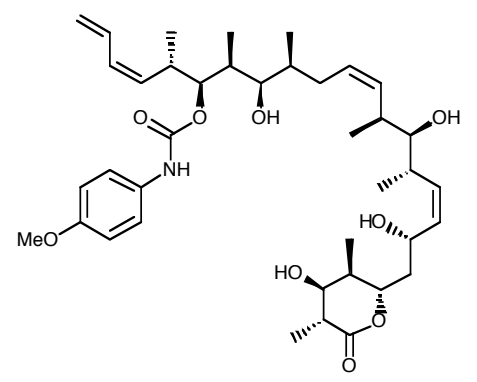

$(+)-9$

p-Methoxyphenyl carbamate tetra-ol (+)-9: Tetra-TBS carbamate (+)-S4 (4.8 mg, $4.21 \mathrm{mmol})$ was dissolved in $\mathrm{MeOH}(2.0 \mathrm{~mL})$. Aqueous $\mathrm{HCl}(6 \mathrm{~N}, 1.5 \mathrm{~mL})$ was added dropwise over $4 \mathrm{hr}$ at room temperature. The reaction mixture was stirred for $4 \mathrm{hr}$ further, diluted with water $(6 \mathrm{~mL})$ and EtOAc $(10 \mathrm{~mL})$. The resulting mixture was neutralized by solid $\mathrm{NaHCO}_{3}$, and separated. The aqueous layer was extracted with EtOAc $(3 \times 8 \mathrm{~mL})$. The combined organic layers were washed 
with saturated brine $(1 \times 15 \mathrm{~mL})$, dried over $\mathrm{Na}_{2} \mathrm{SO}_{4}$, filtered, and concentrated. Preparative TLC (9\% $\left.\mathrm{MeOH} / \mathrm{CH}_{2} \mathrm{Cl}_{2}\right)$ provided $2.8 \mathrm{mg}$-methoxyphenyl carbamate tetra-ol (+)-9 (97\%) as a white solid. $[\square]_{\mathrm{D}}^{23}+41^{\circ}\left(c\right.$ 0.2, MeOH); IR (film, NaCl) 3430, 2970, 1704, 1515, 1227, $1031 \mathrm{~cm}^{-1} ;{ }^{1} \mathrm{H}$ NMR (500 MHz, CD 3 CN) $\square 7.53(\mathrm{br} \mathrm{s}, 1 \mathrm{H}), 7.28(\mathrm{~d}, J=8.2 \mathrm{~Hz}, 2 \mathrm{H}), 6.85(\mathrm{~d}, J=8.9 \mathrm{~Hz}, 2 \mathrm{H}), 6.69$ (ddd, $J=16.7,10.6,10.6 \mathrm{~Hz}, 1 \mathrm{H}), 6.04($ app t, $J=11.0 \mathrm{~Hz}, 1 \mathrm{H}), 5.44(\mathrm{~m}, 2 \mathrm{H}), 5.37$ (dd, $J=11.1$, 8.3 Hz, 1H), $5.22(\mathrm{~m}, 3 \mathrm{H}), 5.13(\mathrm{~d}, J=10.1 \mathrm{~Hz}, 1 \mathrm{H}), 4.80($ app t, $J=6.0 \mathrm{~Hz}, 1 \mathrm{H}), 4.51(\mathrm{~m}, 1 \mathrm{H})$, 4.44 (ddd, $J=10.4,10.4,1.8 \mathrm{~Hz}, 1 \mathrm{H}), 3.74(\mathrm{~s}, 3 \mathrm{H}), 3.60$ (dd, $J=8.2,4.1 \mathrm{~Hz}, 1 \mathrm{H}), 3.28(\mathrm{~d}, J=4.5$ Hz, 1H), $3.23(\mathrm{dd}, J=11.3,6.2 \mathrm{~Hz}, 1 \mathrm{H}), 3.12(\mathrm{~m}, 2 \mathrm{H}), 2.82(\mathrm{~d}, J=5.0 \mathrm{~Hz}, 1 \mathrm{H}), 2.62(\mathrm{~m}, 2 \mathrm{H}), 2.59$ (d, $J=6.6 \mathrm{~Hz}, 1 \mathrm{H}), 2.55$ (ddd, $J=14.6,7.4,4.6 \mathrm{~Hz}, 1 \mathrm{H}), 2.42(\mathrm{~m}, 1 \mathrm{H}), 1.83(\mathrm{~m}, 2 \mathrm{H}), 1.71(\mathrm{~m}$, 3H), 1.54 (ddd, $J=13.8,10.6,2.5 \mathrm{~Hz}, 1 \mathrm{H}), 1.18(\mathrm{~d}, J=7.4 \mathrm{~Hz}, 3 \mathrm{H}), 0.99(\mathrm{~d}, J=6.9 \mathrm{~Hz}, 3 \mathrm{H}), 0.98$ (d, $J=6.7 \mathrm{~Hz}, 3 \mathrm{H}), 0.96(\mathrm{~d}, J=7.0 \mathrm{~Hz}, 3 \mathrm{H}), 0.90(\mathrm{~d}, J=6.7 \mathrm{~Hz}, 3 \mathrm{H}), 0.89(\mathrm{~d}, J=6.7 \mathrm{~Hz}, 3 \mathrm{H})$, $0.84(\mathrm{~d}, J=6.6 \mathrm{~Hz}, 3 \mathrm{H}) ;{ }^{13} \mathrm{C}$ NMR $\left(125 \mathrm{MHz}, \mathrm{CD}_{3} \mathrm{CN}\right) \square 174.7,156.6,155.4,135.0,134.6$, 134.0, 133.6, 133.4, 133.0, 130.5, 128.3, 121.4, 118.4, 114.9, 79.3, 79.2, 78.0, 75.3, 73.1, 63.8, $56.0,44.0,42.3,38.4,36.7,36.6,36.3,36.2,35.0,32.3,19.1,18.1,16.6,15.7,15.3,13.1,9.6$; high resolution mass spectrum $\left(\mathrm{ES}^{+}\right) \mathrm{m} / 2708.4101 \quad\left[(\mathrm{M}+\mathrm{Na})^{+}\right.$; calcd for $\mathrm{C}_{39} \mathrm{H}_{59} \mathrm{NO}{ }_{9} \mathrm{Na}$ : 708.4088].

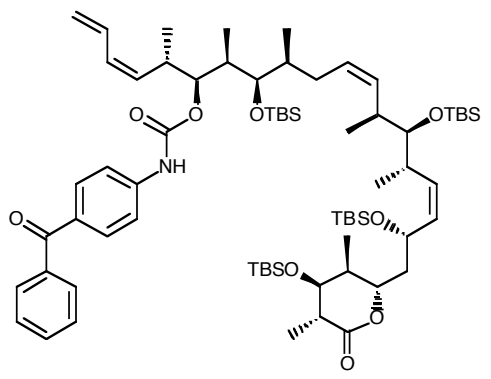

$(+)-\mathrm{S} 5$

Benzophenone carbamate (+)-S5: Preparation for ca. $0.2 \mathrm{M}$ benzophenone isocyanate in benzene solution: A solution of 4-benzoyl-4-benzoic acid (121.8 $\mathrm{mg}, 0.538 \mathrm{mmol})$ in benzene (2.49 $\mathrm{mL})$ was treated with diphenylphosphoryl azide $(119.2 \mathrm{~mL}, 0.555 \mathrm{mmol})$ and triethyl amine 
(77.9 mL, $0.555 \mathrm{mmol}$ ) at $85{ }^{\circ} \mathrm{C}$ for $2 \mathrm{hr}$ to give ca. $0.2 \mathrm{M}$ benzophenone isocyanate in benzene solution.

Carbamate formation: A solution of alcohol (+)-5 (10.0 mg, $10.0 \mathrm{mmol})$ was dissolved in ca. 0.2 $\mathrm{M}$ benzophenone isocyanate solution, warmed to $45^{\circ} \mathrm{C}$, and stirred for $1 \mathrm{hr} 35 \mathrm{~min}$. The reaction mixture was quenched with saturated $\mathrm{NH}_{4} \mathrm{Cl}(3 \mathrm{~mL})$, and diluted with $\mathrm{Et}_{2} \mathrm{O}(6 \mathrm{~mL})$. The resulting layers were separated, and the organic layer was washed with saturated $\mathrm{NH}_{4} \mathrm{Cl}(2 \times 10 \mathrm{~mL})$, saturated $\mathrm{NaHCO}_{3}(1 \times 10 \mathrm{~mL})$ and saturated brine $(1 \times 10 \mathrm{~mL})$, dried over $\mathrm{Na}_{2} \mathrm{SO}_{4}$, filtered, and concentrated. Preparative TLC (9\% ethyl acetate/hexanes) gave $10.9 \mathrm{mg}$ tetra-TBS carbamate (+)-S5 (89\%) as a colorless solid. [ $[\square]_{\mathrm{D}}^{23}+78\left(c\right.$ 0.09, $\left.\mathrm{CHCl}_{3}\right)$; IR (film, $\left.\mathrm{NaCl}\right) 3323,2957,1739$, 1217, 1046, $836 \mathrm{~cm}^{-1} ;{ }^{1} \mathrm{H}$ NMR $\left(500 \mathrm{MHz}, \mathrm{CHCl}_{3}\right) \square 7.78(\mathrm{~d}, J=8.7 \mathrm{~Hz}, 2 \mathrm{H}), 7.74(\mathrm{~d}, J=8.3 \mathrm{~Hz}$, 2H), 7.55 (app t, $J=7.5 \mathrm{~Hz}, 1 \mathrm{H}), 7.45(\mathrm{~m}, 4 \mathrm{H}), 6.85$ (br s, 1H), 6.60 (ddd, $J=16.9,10.7,10.7 \mathrm{~Hz}$, 1H), $6.01(\operatorname{app~t}, J=11.0 \mathrm{~Hz}, 1 \mathrm{H}), 5.38(\operatorname{app~t}, J=10.5 \mathrm{~Hz}, 1 \mathrm{H}), 5.10-5.35(\mathrm{~m}, 6 \mathrm{H}), 4.83(\operatorname{app~t}, J$ $=6.0 \mathrm{~Hz}, 1 \mathrm{H}), 4.78(\operatorname{app} t, J=8.9 \mathrm{~Hz}, 1 \mathrm{H}), 4.50(\operatorname{app~t}, J=10.3 \mathrm{~Hz}, 1 \mathrm{H}), 3.62(\operatorname{app~t}, J=2.3 \mathrm{~Hz}$, 1H), $3.50(\operatorname{app~t}, J=4.4 \mathrm{~Hz}, 1 \mathrm{H}), 3.28(\operatorname{app~t}, J=5.0 \mathrm{~Hz}, 1 \mathrm{H}), 3.04(\operatorname{app~dq}, J=16.7,6.6 \mathrm{~Hz}, 1 \mathrm{H})$, $2.60(\mathrm{~m}, 2 \mathrm{H}), 2.53(\mathrm{~m}, 1 \mathrm{H}), 1.92(\mathrm{~m}, 3 \mathrm{H}), 1.79(\mathrm{ddd}, J=9.7,6.7,2.1 \mathrm{~Hz}, 1 \mathrm{H}), 1.70(\mathrm{~m}, 3 \mathrm{H}), 1.21$ (d, $J=7.6 \mathrm{~Hz}, 3 \mathrm{H}), 1.03(\mathrm{~d}, J=6.8 \mathrm{~Hz}, 3 \mathrm{H}), 0.96(\mathrm{~d}, J=7.2 \mathrm{~Hz}, 3 \mathrm{H}), 0.95(\mathrm{~d}, J=7.2 \mathrm{~Hz}, 3 \mathrm{H})$, $0.94(\mathrm{~d}, J=6.8 \mathrm{~Hz}, 3 \mathrm{H}), 0.91(\mathrm{~s}, 9 \mathrm{H}), 0.88(\mathrm{~s}, 9 \mathrm{H}), 0.88$ (d, obscured, 3H), $0.86(\mathrm{~s}, 9 \mathrm{H}), 0.84(\mathrm{~s}$, 9H), $0.82(\mathrm{~d}, J=6.7 \mathrm{~Hz}, 3 \mathrm{H}), 0.084(\mathrm{~s}, 3 \mathrm{H}), 0.051(\mathrm{~s}, 3 \mathrm{H}), 0.046(\mathrm{~s}, 6 \mathrm{H}), 0.036(\mathrm{~s}, 3 \mathrm{H}), 0.027$ (s, 3H), $\left(0.018(\mathrm{~s}, 3 \mathrm{H}),-0.006(\mathrm{~s}, 3 \mathrm{H}) ;{ }^{13} \mathrm{C}\right.$ NMR $\left(125 \mathrm{MHz}, \mathrm{CHCl}_{3}\right) \square 196.0,173.3,153.2,142.2$, 138.0, 135.5, 133.4, 133.2, 132.5, 132.1, 132.1, 132.0, 131.7, 130.1, 129.8, 128.2, 126.9, 118.3, $117.5,80.0,79.8,77.1,76.2,74.8,64.6,44.1,42.8,38.3,37.9,37.6,35.1,34.5,34.2,31.6,26.2$, $26.2,25.9,25.7,18.5,18.4,17.9,17.8,17.6,17.3,16.4,15.8,14.6,10.3,-3.1,-3.5,-3.6,-4.2,-$ 4.4, -4.5, -4.9; high resolution mass spectrum $\left(\mathrm{ES}^{+}\right) \mathrm{m} / \mathrm{z} 1238.7687\left[(\mathrm{M}+\mathrm{Na})^{+}\right.$; calcd for $\left.\mathrm{C}_{69} \mathrm{H}_{117} \mathrm{NO}_{9} \mathrm{NaSi}_{4}: 1238.7703\right]$ 




$(+)-10$

Benzophenone carbamate tetra-ol (+)-10. Carbamate (+)-S5 (10.9 mg, $0.009 \mathrm{mmol})$ was dissolved in $\mathrm{MeOH}(2.0 \mathrm{~mL})$ and stirred for $15 \mathrm{~min}$ at room temperature. Aqueous hydrochloric acid $(3 \mathrm{~N}, 5.0 \mathrm{~mL}$ ) was added in $200-400 \mu \mathrm{L}$ portions over 4 hours at a rate which minimized precipitation (ca. 10 to $15 \mathrm{~min}$ intervals). An additional $2 \mathrm{~mL}$ of $3 \mathrm{~N}$ aq $\mathrm{HCl}$ was added over $1 \mathrm{~h}$ at $15 \mathrm{~min}$ intervals, and the sides of the flask/stir bar were rinsed with $2.0 \mathrm{~mL}$ of $\mathrm{MeOH}$. After $12 \mathrm{~h}$ the solution was quenched with $\mathrm{NaHCO}_{3}(\mathrm{~s})$, diluted with $30 \mathrm{~mL}$ of water and extracted $3 \times$ with EtOAc. The combined organic extracts were dried $\left(\mathrm{Na}_{2} \mathrm{SO}_{4}\right)$, filtered, and concentrated. Preparative TLC $\left(80 \%\right.$ ethyl acetate/ $\left.\mathrm{CH}_{2} \mathrm{Cl}_{2}\right)$ afforded $(+)-10(5.3 \mathrm{mg}, 78 \%$ yield $) . \quad[\square]_{D}^{23}+36(c$ 0.18, MeOH); IR (film, NaCl) 3398, 2926, 1701, 1596, 1221, $1044 \mathrm{~cm}^{-1} ;{ }^{1} \mathrm{H}$ NMR (500 MHz, $\left.\mathrm{CD}_{3} \mathrm{CN}\right) \square 8.13$ (br s, $\left.1 \mathrm{H}\right), 7.77(\mathrm{dd}, J=6.9,1.9 \mathrm{~Hz}, 2 \mathrm{H}), 7.74(\mathrm{dd}, J=8.3,1.1 \mathrm{~Hz}, 2 \mathrm{H}), 7.63(\mathrm{~m}$, 1H), $7.57(\mathrm{~d}, J=8.7 \mathrm{~Hz}, 2 \mathrm{H}), 7.53(\operatorname{app~t}, J=7.7 \mathrm{~Hz}, 2 \mathrm{H}), 6.72(\mathrm{ddd}, J=17.0,10.5,10.5 \mathrm{~Hz}, 1 \mathrm{H})$, $6.07(\operatorname{app~t}, J=11.0 \mathrm{~Hz}, 1 \mathrm{H}), 5.45(\mathrm{~m}, 2 \mathrm{H}), 5.38(\mathrm{dd}, J=11.1,8.2 \mathrm{~Hz}, 1 \mathrm{H}), 5.23(\mathrm{~m}, 2 \mathrm{H}), 5.16(\mathrm{~d}$, $J=10.1 \mathrm{~Hz}, 1 \mathrm{H}), 4.87(\operatorname{app~t}, J=6.0 \mathrm{~Hz}, 1 \mathrm{H}), 4.52(\mathrm{~m}, 1 \mathrm{H}), 4.46(\mathrm{ddd}, J=10.5,10.5,1.9 \mathrm{~Hz}$, 1H), $3.61(\mathrm{~m}, 1 \mathrm{H}), 3.30(\mathrm{~m}, 1 \mathrm{H}), 3.26(\mathrm{dd}, J=11.4,6.3 \mathrm{~Hz}, 1 \mathrm{H}), 3.15(\mathrm{~m}, 1 \mathrm{H}), 2.84(\mathrm{~d}, J=4.9 \mathrm{~Hz}$, 1H), $2.64(\mathrm{~m}, 2 \mathrm{H}), 2.56$ (ddd, $J=14.7,7.4,4.7 \mathrm{~Hz}, 1 \mathrm{H}$ ), 2.44 (app dq, $J=15.1,6.7 \mathrm{~Hz}, 1 \mathrm{H}), 1.82-$ $1.90(\mathrm{~m}, 2 \mathrm{H}), 1.77(\mathrm{~m}, 1 \mathrm{H}), 1.72(\mathrm{ddd}, J=14.3,10.5,2.1 \mathrm{~Hz}, 1 \mathrm{H}), 1.66(\mathrm{~m}, 1 \mathrm{H}), 1.55,(\mathrm{ddd}, J=$ 14.3, 10.6, $2.5 \mathrm{~Hz}, 1 \mathrm{H}), 1.19(\mathrm{~d}, J=7.4 \mathrm{~Hz}, 3 \mathrm{H}), 1.01(\mathrm{~d}, J=6.9 \mathrm{~Hz}, 3 \mathrm{H}), 1.00(\mathrm{~d}, J=7.0 \mathrm{~Hz}, 3 \mathrm{H})$, $0.97(\mathrm{~d}, J=6.9 \mathrm{~Hz}, 3 \mathrm{H}), 0.95(\mathrm{~d}, J=6.8 \mathrm{~Hz}, 3 \mathrm{H}), 0.90(\mathrm{~d}, J=6.7 \mathrm{~Hz}, 3 \mathrm{H}), 0.86(\mathrm{~d}, J=6.6 \mathrm{~Hz}$, $3 \mathrm{H}) ;{ }^{13} \mathrm{C}$ NMR $\left(125 \mathrm{MHz}, \mathrm{CD}_{3} \mathrm{CN}\right) \square 196.0,174.8,154.7,144.4,139.1,135.0,134.4,134.0$, $133.7,133.3,133.0,132.4,132.3,130.7,130.4,129.2,128.2,118.5,117.5,80.0,79.3,78.0$, 
$75.2,73.1,63.8,43.9,42.3,38.3,36.7,36.6,36.3,35.0,32.2,28.5,19.1,18.0,16.6,15.7,15.2$, 13.0, 9.6; high resolution mass spectrum (ES $\left.{ }^{+}\right) \mathrm{m} / 2$ 782.4222 $\left[(\mathrm{M}+\mathrm{Na})^{+}\right.$; calcd for $\mathrm{C}_{45} \mathrm{H}_{61} \mathrm{NO}_{9} \mathrm{Na}$ : 782.4244].

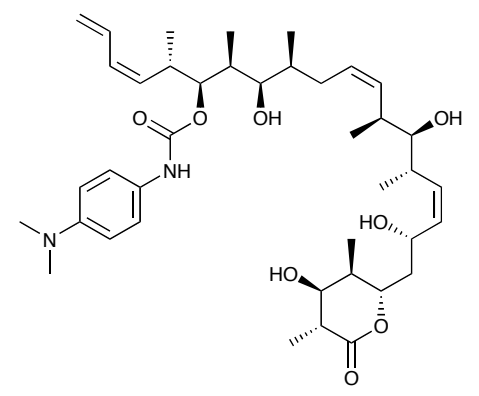

$(+)-11$

Dimethylaniline carbamate tetra-ol (+)-11: A solution of alcohol (+)-5 (18 $\mathrm{mg}, 0.018 \mathrm{mmol})$ in pyridine $(0.83 \mathrm{~mL})$ was treated with $\mathrm{N}, \mathrm{N}$-dimethylaminophenyl isocyanate $(55 \mathrm{mg}, 0.339 \mathrm{mmol})$ at room temperature for $24 \mathrm{hr}$. The reaction mixture was quenched with saturated $\mathrm{NH}_{4} \mathrm{Cl}(2 \mathrm{~mL})$ and diluted with $\mathrm{CH}_{2} \mathrm{Cl}_{2}(5 \mathrm{~mL})$. The resulting layers were separated, and the aqueous layer was extracted with $\mathrm{CH}_{2} \mathrm{Cl}_{2}(3 \times 5 \mathrm{~mL})$. The combined organic layers were washed with saturated brine (1 $\times 8 \mathrm{~mL}$ ), dried over $\mathrm{Na}_{2} \mathrm{SO}_{4}$, filtered, and concentrated, and quickly flushed through a plug of silica gel to remove the excess reagent. The resulting tetra-TBS carbamate residue was dissolved in $\mathrm{MeOH}(4.0 \mathrm{~mL})$. Aqueous $\mathrm{HCl}(4 \mathrm{~N}, 3.1 \mathrm{~mL})$ was added dropwise over $4 \mathrm{hr}$ at room temperature. The reaction mixture was stirred for $4 \mathrm{hr}$ further, diluted with water $(10 \mathrm{~mL})$ and EtOAc $(15 \mathrm{~mL})$. The resulting mixture was neutralized with solid $\mathrm{NaHCO}_{3}$, and the layers separated. The aqueous layer was extracted with EtOAc $(3 \times 8 \mathrm{~mL})$. The combined organic layers were washed with saturated brine $(1 \times 15 \mathrm{~mL})$, dried over $\mathrm{Na}_{2} \mathrm{SO}_{4}$, filtered, and concentrated. Flash chromatography $\left(5 \% \mathrm{MeOH} / \mathrm{CH}_{2} \mathrm{Cl}_{2}\right)$ provided dimethylaniline carbamoyl tetra-ol (+)-11 (9 mg, 72\%) as a colorless oil. $[\square]_{D}^{23}+45.0(c 0.33, \mathrm{MeOH})$; IR (film, $\left.\mathrm{NaCl}\right) 3436,2969,2933,2878$, $1704,1593,1525,1456,1375,1320,1238,1100,1045,973,948 \mathrm{~cm}^{-1} ;{ }^{1} \mathrm{H}$ NMR $(500 \mathrm{MHz}$, $\left.\mathrm{CD}_{3} \mathrm{OD}\right) \square 7.21$ (br s, 2H), 6.80-6.70 (m, 2H), 6.67 (ddd, $\left.J=16.7,10.4,10.4 \mathrm{~Hz}, 1 \mathrm{H}\right), 6.03$ (dd, $J=$ $11.2,10.8 \mathrm{~Hz}, 1 \mathrm{H}), 5.51(\mathrm{dd}, J=10.8,10.8 \mathrm{~Hz}, 1 \mathrm{H}), 5.52-5.43(\mathrm{~m}, 1 \mathrm{H}), 5.36(\mathrm{dd}, J=10.8,8.9 \mathrm{~Hz}$, 
1H), 5.27-5.18 (m, 3H), $5.12(\mathrm{~d}, J=10.4 \mathrm{~Hz}, 1 \mathrm{H}), 4.88(\mathrm{dd}, J=6.3,4.8 \mathrm{~Hz}, 1 \mathrm{H}), 4.62(\mathrm{dd}, J=$ $10.1,10.1 \mathrm{~Hz}, 1 \mathrm{H}), 4.55$ (ddd, $J=10.4,10.4,1.9 \mathrm{~Hz}, 1 \mathrm{H}), 3.58(\mathrm{dd}, J=4.1,3.7 \mathrm{~Hz}, 1 \mathrm{H}), 3.28-$ $3.25(\mathrm{~m}, 1 \mathrm{H}), 3.15(\mathrm{dd}, J=7.8,3.7 \mathrm{~Hz}, 1 \mathrm{H}), 3.15-3.10(\mathrm{~m}, 1 \mathrm{H}), 2.85(\mathrm{br} \mathrm{s}, 6 \mathrm{H}), 2.73-2.64(\mathrm{~m}, 1 \mathrm{H})$, 2.60 (dddd, $J=7.4,7.4,7.4,4.1 \mathrm{~Hz}, 1 \mathrm{H}$ ), 2.42 (dddd, $J=14.9,7.8,6.7,6.7 \mathrm{~Hz}, 1 \mathrm{H}$ ), 1.95-1.70 (m, 5H), 1.70-1.62 (m, 1H), 1.57 (ddd, $J=14.5,10.8,2.2 \mathrm{~Hz}, 1 \mathrm{H}), 1.22(\mathrm{~d}, J=7.4 \mathrm{~Hz}, 3 \mathrm{H}), 1.04$ $(\mathrm{d}, J=7.1 \mathrm{~Hz}, 3 \mathrm{H}), 1.02-0.98(\mathrm{~m}, 6 \mathrm{H}), 0.94(\operatorname{app~d}, J=6.7 \mathrm{~Hz}, 6 \mathrm{H}), 0.87(\mathrm{~d}, J=6.7 \mathrm{~Hz}, 3 \mathrm{H}) ;{ }^{13} \mathrm{C}$ NMR $\left(125 \mathrm{MHz}, \mathrm{CD}_{3} \mathrm{OD}\right) \square 175.5,155.4,147.4,133.6,132.9,132.4,132.2,132.0$ (2), 129.5, $127.1,120.5,116.9,113.6,78.8,78.4,77.5,74.5,72.1,62.6,43.0,41.3,40.2,37.5,35.7,35.6$, $35.5,35.2,33.8,31.2,17.9,16.8,15.9,14.5,14.2,11.8,8.4$; high resolution mass spectrum $\left(\mathrm{ES}^{+}\right) \mathrm{m} / \mathrm{z} 721.4393\left[(\mathrm{M}+\mathrm{Na})^{+}\right.$; calcd for $\left.\mathrm{C}_{40} \mathrm{H}_{62} \mathrm{~N}_{2} \mathrm{O}_{8} \mathrm{Na}: 721.4404\right]$.

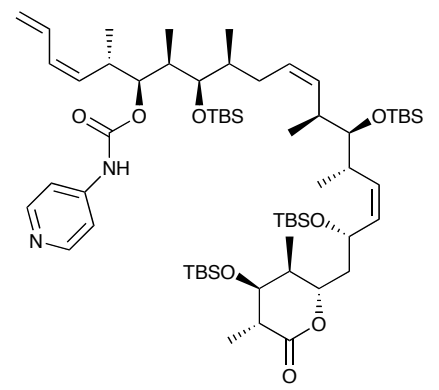

$(+)-\mathrm{S} 6$

Pyridine Carbamate (+)-S6. Preparation for ca. $0.2 \mathrm{M}$ pyridyl isocyanate in benzene solution: A solution of isonicotinic acid $(2.0 \mathrm{~g}, 16.3 \mathrm{mmol})$ in DMF $(12 \mathrm{~mL})$ was treated with diphenylphosphoryl azide $(3.5 \mathrm{~mL}, 16.3 \mathrm{mmol})$ and triethyl amine $(2.27 \mathrm{~mL}, 16.3 \mathrm{mmol})$ at $35{ }^{\circ} \mathrm{C}$ for $2 \mathrm{hr}$. Quenched by pouring reaction mixture into $\mathrm{Et}_{2} \mathrm{O} / \mathrm{ice}$, extracted $2 \times 30 \mathrm{~mL}$ ether. Washed combined organics with saturated $\mathrm{NaHCO}_{3}(15 \mathrm{~mL})$ and water $(15 \mathrm{~mL})$. Dried over $\mathrm{MgSO}_{4}$, concentrated and redissolved in benzene. Heated solution to $80^{\circ} \mathrm{C}$ for $1.5 \mathrm{~h}$. and then cooled to room temperature to yield a $0.2 \mathrm{M}$ pyridyl isocyanate in benzene solution.

Carbamate formation: A solution of alcohol $(+)-5(22.0 \mathrm{mg}, 0.022 \mathrm{mmol})$ was dissolved in the above $0.2 \mathrm{M}$ pyridyl isocyanate solution $(1.5 \mathrm{~mL})$, warmed to $50{ }^{\circ} \mathrm{C}$, and stirred for $6 \mathrm{~h}$. The reaction mixture was applied directly to flash chromotography (10\% to $40 \%$ ethyl acetate/hexanes) to yield $8.5 \mathrm{mg}$ tetra-TBS carbamate (+)-S6 (35\%) as a colorless oil in addition 
to recovered (+)-5 (13.0 mg, 36\%). $[\square]_{D}^{23}+44.4\left(\mathrm{c} 0.5, \mathrm{CHCl}_{3}\right)$; IR ( $\left.\mathrm{NaCl}\right) 3325,2957,2929$, 2884, 2857, 1739, 1652, 1594, 1521, 1472, 1462, 1250, 1223, $1207 \mathrm{~cm}^{-1}$; ${ }^{1} \mathrm{H}-\mathrm{NMR}(500 \mathrm{MHz}$, $\left.\mathrm{CDCl}_{3}\right) \square 8.44(\mathrm{br} \mathrm{d}, J=4.1 \mathrm{~Hz}, 2 \mathrm{H}), 7.31(\mathrm{br} \mathrm{d}, J=4.1 \mathrm{~Hz}, 2 \mathrm{H}), 6.91(\mathrm{~s}, 1 \mathrm{H}), 6.60$ (ddd, $J=16.8$, 10.8, 10.8 Hz, 1H), 6.02 (dd, $J=11.2,11.2 \mathrm{~Hz}, 1 \mathrm{H}$ ), 5.36 (dd, $J=10.8,10.8 \mathrm{~Hz}, 1 \mathrm{H}$ ), 5.34-5.20 (m, 4H), 5.20-5.12 (m, 2H), $4.84(\mathrm{dd}, J=5.9,5.9 \mathrm{~Hz}, 1 \mathrm{H}), 4.80(\mathrm{dd}, J=9.7,8.6 \mathrm{~Hz}, 1 \mathrm{H}), 4.52(\mathrm{dd}$, $J=10.4,10.4 \mathrm{~Hz}, 1 \mathrm{H}), 3.64(\mathrm{dd}, J=2.6,2.6 \mathrm{~Hz}, 1 \mathrm{H}), 3.50(\mathrm{dd}, J=4.5,4.5 \mathrm{~Hz}, 1 \mathrm{H}), 3.30(\mathrm{dd}, J=$ 5.6, 4.1 Hz, 1H), 3.05 (ddd, $J=10.0,6.7,6.7 \mathrm{~Hz}, 1 \mathrm{H}), 2.65-2.57(\mathrm{~m}, 2 \mathrm{H}), 2.54$ (dddd, $J=10.8$, 10.8, 6.7, $6.7 \mathrm{~Hz}, 1 \mathrm{H}), 2.01-1.82(\mathrm{~m}, 3 \mathrm{H}), 1.82-1.74(\mathrm{~m}, 1 \mathrm{H}), 1.76-1.68(\mathrm{~m}, 1 \mathrm{H}), 1.73-1.63(\mathrm{~m}$, 1H), 1.60 (dddd, $J=11.2,6.7,6.7,1.9 \mathrm{~Hz}, 1 \mathrm{H}), 1.23(\mathrm{~d}, J=7.5 \mathrm{~Hz}, 3 \mathrm{H}), 1.03(\mathrm{~d}, J=6.7 \mathrm{~Hz}, 3 \mathrm{H})$, 0.96 (app d, $J=6.7 \mathrm{~Hz}, 6 \mathrm{H}$ ), 0.93 (s, 9H), 0.92 (d, obscured, 3H), 0.90 (s, 9H), $0.88(\mathrm{~s}, 9 \mathrm{H}), 0.87$ (d, obscured, 3H), 0.85 (s, 9H), 0.84 (d, J = 6.7 Hz, 3H), 0.09 (s, 3H), 0.070 (s, 3H), 0.068 (app s, 6H), 0.058 (s, 3H), 0.054 (s, 3H), 0.04 (s, 3H), 0.01 (s, 3H); ${ }^{13} \mathrm{C}-\mathrm{NMR}\left(125 \mathrm{MHz}, \mathrm{CDCl}_{3}\right.$ ) $\mathrm{C}$ 173.3, $152.8,150.5,145.3,135.5,133.5,133.0,132.4,131.9,130.1,126.7,118.2,112.4,80.0,79.9$, $77.1,76.1,74.8,64.6,44.0,42.8,32.2,37.8,37.5,35.1,34.4,34.1,31.6,26.2,26.1,25.8,25.6$, $18.5,18.4,18.0,17.9,17.5,17.3,16.3,15.7,14.5,14.0,10.2,-3.2,-3.5,-3.7,-4.3,-4.4,-4.6,-4.9$ (2); high resolution mass spectrum $\left(\mathrm{ES}^{+}\right) \mathrm{m} / \mathrm{z} 1135.7421\left[(\mathrm{M}+\mathrm{Na})^{+}\right.$; calcd for $\mathrm{C}_{61} \mathrm{H}_{112} \mathrm{~N}_{2} \mathrm{O}_{8} \mathrm{Si}_{4} \mathrm{Na}$ : $1135.7394]$

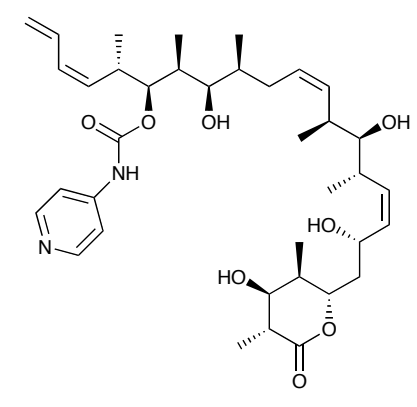

$(+)-12$

Pyridine carbamate tetra-ol (+)-12: Tetra-TBS carbamate (+)-S6 (8.5 mg, $8.0 \mu \mathrm{mol})$ was dissolved in $\mathrm{MeOH}(4.0 \mathrm{~mL})$. Aqueous $\mathrm{HCl}(4 \mathrm{~N}, 3.1 \mathrm{~mL})$ was added dropwise over $4 \mathrm{hr}$ at room temperature. The reaction mixture was stirred for $4 \mathrm{hr}$ further, diluted with water $(10 \mathrm{~mL})$ and 
EtOAc $(15 \mathrm{~mL})$. The resulting mixture was neutralized by solid $\mathrm{NaHCO}_{3}$, and separated. The aqueous layer was extracted with EtOAc $(3 \times 10 \mathrm{~mL})$. The combined organic layers were washed with saturated brine $(1 \times 15 \mathrm{~mL})$, dried over $\mathrm{MgSO}_{4}$, filtered, and concentrated. Flash chromatography (5\% $\left.\mathrm{MeOH} / \mathrm{CH}_{2} \mathrm{Cl}_{2}\right)$ provided pyridine carbamoyl tetra-ol (+)-12 (4.5 mg, 96\%) as a colorless oil. $[\square]_{D}^{23}+36.0(c$ 0.33, $\mathrm{MeOH})$; IR (film, NaCl) 3313, 2968, 2930, 2878, 1718, $1595,1521,1458,1420,1374,1333,1247,1207,1099,1045 \mathrm{~cm}^{-1} ;{ }^{1} \mathrm{H}$ NMR $\left(500 \mathrm{MHz}, \mathrm{CD}_{3} \mathrm{OD}\right)$ $\square 8.31(\mathrm{~d}, J=6.3 \mathrm{~Hz}, 2 \mathrm{H}), 7.48(\mathrm{dd}, J=6.3,4.8 \mathrm{~Hz}, 2 \mathrm{H}), 6.67(\mathrm{ddd}, J=16.7,10.8,10.8 \mathrm{~Hz}, 1 \mathrm{H})$, $6.03(\mathrm{dd}, J=10.8,10.8 \mathrm{~Hz}, 1 \mathrm{H}), 5.50(\mathrm{dd}, J=10.1,10.1 \mathrm{~Hz}, 1 \mathrm{H}), 5.44(\mathrm{dd}, J=10.8,10.8 \mathrm{~Hz}$, 1H), $5.36(\mathrm{dd}, J=10.8,8.6 \mathrm{~Hz}, 1 \mathrm{H}), 5.26-5.17(\mathrm{~m}, 3 \mathrm{H}), 5.13(\mathrm{~d}, J=10.1 \mathrm{~Hz}, 1 \mathrm{H}), 4.94(\mathrm{dd}, J=$ 7.1, $5.2 \mathrm{~Hz}, 1 \mathrm{H}$ ), $4.62(\mathrm{dd}, J=9.3,9.3 \mathrm{~Hz}, 1 \mathrm{H}), 4.55$ (ddd, $J=10.4,10.4,1.8 \mathrm{~Hz}, 1 \mathrm{H}), 3.58(\mathrm{dd}, J$ $=4.1,3.7 \mathrm{~Hz}, 1 \mathrm{H}), 3.30-3.25(\mathrm{~m}, 1 \mathrm{H}), 3.17(\mathrm{ddd}, J=17.9,6.3,6.3 \mathrm{~Hz}, 1 \mathrm{H}), 3.14(\mathrm{dd}, J=7.4,4.1$ Hz, 1H), 2.71-2.63 (m, 1H), 2.60 (dddd, $J=7.4,7.4,7.4,4.5 \mathrm{~Hz}, 1 \mathrm{H}), 2.42$ (dddd, $J=14.4,7.1$, 7.1, $7.1 \mathrm{~Hz}, 1 \mathrm{H}), 1.94-1.88(\mathrm{~m}, 2 \mathrm{H}), 1.85-1.70(\mathrm{~m}, 3 \mathrm{H}), 1.70-1.63(\mathrm{~m}, 1 \mathrm{H}), 1.58$ (ddd, $J=14.4$, 10.8, 2.2 Hz, 1H), $1.23(\mathrm{~d}, J=7.4 \mathrm{~Hz}, 3 \mathrm{H}), 1.03(\mathrm{~d}, J=7.1 \mathrm{~Hz}, 3 \mathrm{H}), 1.01(\mathrm{~d}, J=6.7 \mathrm{~Hz}, 3 \mathrm{H}), 1.00$ $(\mathrm{d}, J=6.7 \mathrm{~Hz}, 3 \mathrm{H}), 0.96(\mathrm{~d}, J=6.7 \mathrm{~Hz}, 3 \mathrm{H}), 0.92(\mathrm{~d}, J=6.7 \mathrm{~Hz}, 3 \mathrm{H}), 0.88(\mathrm{~d}, J=6.3 \mathrm{~Hz}, 3 \mathrm{H}) ;{ }^{13} \mathrm{C}$ NMR $\left(125 \mathrm{MHz}, \mathrm{CD}_{3} \mathrm{OD}\right) \square$ 175.5, 153.9, 149.1, 147.5, 133.7, 132.5 (2), 132.2, 131.9, 129.8, $126.9,117.2,112.5,79.4,78.7,77.5,74.3,72.1,62.6,43.0,41.3,37.3,35.7,35.6,35.5,35.2$, $33.8,31.1,17.8,16.7,15.9,14.5,14.1,11.8,8.4 ;$ high resolution mass spectrum $\left(\mathrm{ES}^{+}\right) \mathrm{m} / \mathrm{z}$ $679.3950\left[(\mathrm{M}+\mathrm{Na})^{+}\right.$; calcd for $\left.\mathrm{C}_{37} \mathrm{H}_{56} \mathrm{~N}_{2} \mathrm{O}_{8} \mathrm{Na:} 679.3934\right]$.

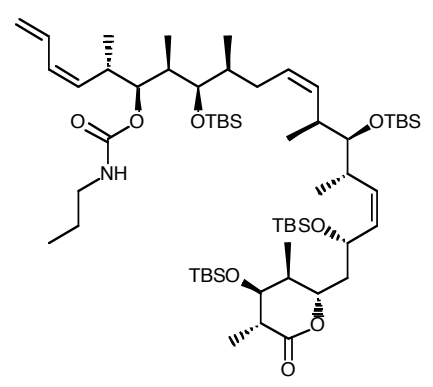

(+)-S7 
n-Propyl carbamate (+)-S7: A solution of alcohol (+)-5 (17.2 mg, $17.3 \mu \mathrm{mol})$ in $\mathrm{CH}_{2} \mathrm{Cl}_{2}(1.73$ $\mathrm{mL}$ ) was treated with $n$-propyl isocyanate $(49 \mu \mathrm{L}, 0.520 \mathrm{mmol})$ and DBU $(39 \mu \mathrm{L}, 0.260 \mathrm{mmol})$ at room temperature for $16 \mathrm{hr}$. The reaction mixture was quenched with saturated $\mathrm{NH}_{4} \mathrm{Cl}(5 \mathrm{~mL})$, diluted with $\mathrm{CH}_{2} \mathrm{Cl}_{2}(5 \mathrm{~mL})$. The resulting layers were separated, and the aqueous layer was extracted with $\mathrm{CH}_{2} \mathrm{Cl}_{2}(3 \times 7 \mathrm{~mL})$. The combined organic layers were washed with saturated $\mathrm{NH}_{4} \mathrm{Cl}(1 \times 10 \mathrm{~mL})$ and saturated brine $(1 \times 10 \mathrm{~mL})$, dried over $\mathrm{Na}_{2} \mathrm{SO}_{4}$, filtered, and concentrated. Flash chromatograpy ( $9 \%$ ethyl acetate/hexanes) gave $18.7 \mathrm{mg}$ tetra-TBS carbamate (+)-S7 $(100 \%)$ as a colorless oil. $[\square]_{D}^{23}+43\left(c 0.30, \mathrm{CHCl}_{3}\right)$; IR (film, $\left.\mathrm{NaCl}\right) 3383$, 2958, 1725, 1698, 1463, 1253, 1047, $836 \mathrm{~cm}^{-1}$; ${ }^{1} \mathrm{H}$ NMR $\left(500 \mathrm{MHz}, \mathrm{CDCl}_{3}\right) \square 6.57$ (ddd, $J=$ 17.0, 10.6, $10.6 \mathrm{~Hz}, 1 \mathrm{H}), 5.98(\operatorname{app~t}, J=11.0 \mathrm{~Hz}, 1 \mathrm{H}), 5.36(\operatorname{app~t}, J=10.5 \mathrm{~Hz}, 1 \mathrm{H}), 5.26(\mathrm{~m}$, 2H), $5.18(\mathrm{~d}, J=18.0 \mathrm{~Hz}, 1 \mathrm{H}), 5.12(\mathrm{~m}, 1 \mathrm{H}), 5.10(\mathrm{~d}, J=10.2 \mathrm{~Hz}, 1 \mathrm{H}), 4.78(\operatorname{app~t}, J=9.0 \mathrm{~Hz}$, 1H), $4.70($ app t, $J=5.3 \mathrm{~Hz}, 1 \mathrm{H}), 4.56(\mathrm{br} \mathrm{s}, 1 \mathrm{H}), 4.49$ (ddd, $J=10.9,10.9,1.1 \mathrm{~Hz}, 1 \mathrm{H}), 3.83(\mathrm{~m}$, 1H), $3.63(\operatorname{app~t}, J=2.4 \mathrm{~Hz}, 1 \mathrm{H}), 3.44(\operatorname{app~t}, J=4.4 \mathrm{~Hz}, 1 \mathrm{H}), 3.27(\operatorname{app~t}, J=5.2 \mathrm{~Hz}, 1 \mathrm{H}), 3.08$ (m, 2H), $2.95(\mathrm{~m}, 2 \mathrm{H}), 2.61(\mathrm{~m}, 2 \mathrm{H}), 2.52(\mathrm{~m}, 1 \mathrm{H}), 1.85(\mathrm{~m}, 2 \mathrm{H}), 1.79$ (dddd, $J=16.8,6.9,6.9,2.0$ $\mathrm{Hz}, 1 \mathrm{H}), 1.72(\mathrm{ddd}, J=14.0,11.0,1.5 \mathrm{~Hz}, 1 \mathrm{H}), 1.66(\mathrm{~m}, 1 \mathrm{H}), 1.56(\mathrm{~m}, 2 \mathrm{H}), 1.47$ (app dq, $J=$ 14.6, $7.3 \mathrm{~Hz}, 1 \mathrm{H}), 1.21(\mathrm{~d}, J=7.6 \mathrm{~Hz}, 3 \mathrm{H}), 0.96(\mathrm{~d}, J=7.9 \mathrm{~Hz}, 3 \mathrm{H}), 0.95(\mathrm{~d}, J=6.8 \mathrm{~Hz}, 6 \mathrm{H}), 0.93$ (d, $J=7.5 \mathrm{~Hz}, 3 \mathrm{H}), 0.90$ (s, 9H), 0.90 (d, obscured, 3H), $0.88(\mathrm{~s}, 9 \mathrm{H}), 0.87$ (d, $J=6.4 \mathrm{~Hz}, 3 \mathrm{H}$ ), $0.86(\mathrm{~s}, 9 \mathrm{H}), 0.85(\mathrm{~s}, 9 \mathrm{H}), 0.78(\mathrm{~d}, J=6.7 \mathrm{~Hz}, 3 \mathrm{H}), 0.073(\mathrm{~s}, 3 \mathrm{H}), 0.053(\mathrm{~s}, 3 \mathrm{H}), 0.050(\mathrm{~s}, 3 \mathrm{H})$, $0.040(\mathrm{~s}, 6 \mathrm{H}), 0.036(\mathrm{~s}, 3 \mathrm{H}), 0.027(\mathrm{~s}, 3 \mathrm{H}),-0.002(\mathrm{~s}, 3 \mathrm{H}) ;{ }^{13} \mathrm{C} \mathrm{NMR}\left(125 \mathrm{MHz}, \mathrm{CDCl}_{3}\right) \square$ 173.3, 156.8, 135.2, 133.9, 133.6, 132.5, 132.2, 129.7, 127.2, 117.7, 80.0, 78.2, 77.0, 76.2, 74.9, 64.6, $44.5,44.2,42.9,38.2,37.5,35.0,34.5,34.2,32.0,26.3,26.2,25.9,25.7,23.3,21.1,18.5,18.4$, $18.1,18.0,17.7,17.1,16.4,16.0,14.4,14.1,11.2,10.2,-3.1,-3.5,-3.6,-4.2,-4.3,-4.5,-4.9 ;$ high resolution mass spectrum $\left.\left(\mathrm{ES}^{+}\right)\right) \mathrm{m} / \mathrm{z} 1100.7562\left[(\mathrm{M}+\mathrm{Na})^{+}\right.$; calcd for $\mathrm{C}_{59} \mathrm{H}_{115} \mathrm{NO}_{8} \mathrm{NaSi}_{4}$ : 1100.7562]. 




$(+)-13$

n-Propyl carbamate tetra-ol (+)-13: Tetra-TBS carbamate (+)-S7 (7.1 mg, $6.59 \mu \mathrm{mol})$ was dissolved in $\mathrm{MeOH}(3.3 \mathrm{~mL})$. Aqueous $\mathrm{HCl}(6 \mathrm{~N}, 2.6 \mathrm{~mL})$ was added dropwise over $4 \mathrm{hr}$ at room temperature. The reaction mixture was stirred for $4 \mathrm{hr}$ further, diluted with water $(6 \mathrm{~mL})$ and EtOAc $(10 \mathrm{~mL})$. The resulting mixture was neutralized by solid $\mathrm{NaHCO}_{3}$, and separated. The aqueous layer was extracted with EtOAc $(3 \times 8 \mathrm{~mL})$. The combined organic layers were washed with saturated brine $(1 \times 15 \mathrm{~mL})$, dried over $\mathrm{Na}_{2} \mathrm{SO}_{4}$, filtered, and concentrated. Preparative TLC $\left(9 \% \mathrm{MeOH} / \mathrm{CH}_{2} \mathrm{Cl}_{2}\right)$ provided propyl carbamate tetra-ol (+)-13 (2.6 mg, 64\%) as a colorless solid. $[\square]_{\mathrm{D}}^{23}+50$ (c $\left.0.125 \mathrm{MeOH}\right)$; IR (film, NaCl) 3398, 2967, 1698, 1457, 1241, $1102 \mathrm{~cm}^{-1} ;{ }^{1} \mathrm{H}$ NMR $\left(500 \mathrm{MHz}, \mathrm{CD}_{3} \mathrm{CN}\right) \square 6.66(\mathrm{ddd}, J=16.8,10.6,10.6 \mathrm{~Hz}, 1 \mathrm{H}), 6.03($ app t, $J=11.0 \mathrm{~Hz}, 1 \mathrm{H}), 5.40$ (m, 4H), $5.22(\mathrm{~m}, 3 \mathrm{H}), 5.12(\mathrm{~d}, J=10.1 \mathrm{~Hz}, 1 \mathrm{H}), 4.69(\operatorname{app~t}, J=5.4 \mathrm{~Hz}, 1 \mathrm{H}), 4.52(\operatorname{app~t}, J=9.8$ Hz, 1H), 4.45 (ddd, J = 10.4, 10.4, $1.9 \mathrm{~Hz}, 1 \mathrm{H}), 3.63(\mathrm{~m}, 1 \mathrm{H}), 3.30$ (br s, 1H), $3.18(\mathrm{~m}, 1 \mathrm{H}), 3.13$ (dd, $J=11.5,5.0 \mathrm{~Hz}, 1 \mathrm{H}), 3.04(\mathrm{~m}, 1 \mathrm{H}), 3.00(\operatorname{app~dq}, J=6.8,6.8 \mathrm{~Hz}, 2 \mathrm{H}), 2.82(\mathrm{~d}, J=4.7 \mathrm{~Hz}$, 1H), $2.63(\mathrm{~m}, 3 \mathrm{H}), 2.56(\mathrm{dddd}, J=7.3,7.3,7.3,4.6 \mathrm{~Hz}, 1 \mathrm{H}), 2.41(\mathrm{~m}, 1 \mathrm{H}), 1.90-1.77(\mathrm{~m}, 4 \mathrm{H}), 1.71$ (ddd, $J=14.4,10.3,2.0 \mathrm{~Hz}, 1 \mathrm{H}), 1.60(\mathrm{~m}, 1 \mathrm{H}), 1.55$ (ddd, $J=14.3,10.6,2.5 \mathrm{~Hz}, 1 \mathrm{H}$ ), 1.44 (dddd, $J=14.0,6.9,6.9,6.9 \mathrm{~Hz}, 2 \mathrm{H}), 1.20(\mathrm{~d}, J=7.4 \mathrm{~Hz}, 3 \mathrm{H}), 1.00(\mathrm{~d}, J=6.9 \mathrm{~Hz}, 3 \mathrm{H}), 0.98(\mathrm{~d}, J=6.9$ $\mathrm{Hz}, 3 \mathrm{H}), 0.94(\mathrm{~d}, J=6.8 \mathrm{~Hz}, 3 \mathrm{H}), 0.90(\mathrm{~d}, J=6.7 \mathrm{~Hz}, 3 \mathrm{H}), 0.86(\mathrm{t}, J=7.4 \mathrm{~Hz}, 3 \mathrm{H}), 0.83(\mathrm{~d}, J=6.4$ $\mathrm{Hz}, 3 \mathrm{H}), 0.82(\mathrm{~d}, J=6.6 \mathrm{~Hz}, 3 \mathrm{H}) ;{ }^{13} \mathrm{C}$ NMR $\left(125 \mathrm{MHz}, \mathrm{CD}_{3} \mathrm{CN}\right) \square$ 174.7, 158.2, 134.9, 134.7, $134.0,133.6,133.4,130.4,128.3,122.0,79.3,78.8,77.9,75.5,73.1,63.8,44.0,43.2,42.3,38.5$, $36.7,36.6,36.3,35.1,32.1,30.6,23.9,19.1,18.2,16.6,15.7,15.5,13.1,11.5,9.4$; high resolution mass spectrum $\left(\mathrm{ES}^{+}\right) \mathrm{m} / 2$ 644.4122 [(M+Na) ${ }^{+}$; calcd for $\left.\mathrm{C}_{35} \mathrm{H}_{59} \mathrm{NO}_{8} \mathrm{Na}: 644.4138\right]$. 


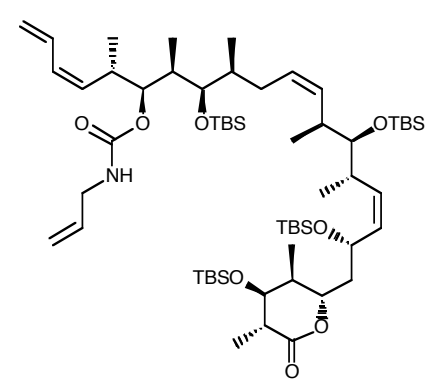

(+)-S8

Allyl carbamate (+)-S8: Alcohol (+)-5 (11.9 mg, $12.0 \mu \mathrm{mol})$ was treated with allyl isocyanate (32 $\mu \mathrm{L}, 0.360 \mathrm{mmol})$ and DBU $(27 \mu \mathrm{L}, 0.180 \mathrm{mmol})$ in $\mathrm{CH}_{2} \mathrm{Cl}_{2}(1.2 \mathrm{~mL})$ at room temperature for $16 \mathrm{~h}$. The reaction mixture was quenched with saturated $\mathrm{NH}_{4} \mathrm{Cl}(5 \mathrm{~mL})$, diluted with $\mathrm{CH}_{2} \mathrm{Cl}_{2}(5 \mathrm{~mL})$. The resulting layers were separated, and the aqueous layer was extracted with $\mathrm{CH}_{2} \mathrm{Cl}_{2}(3 \times 7$ $\mathrm{mL})$. The combined organic layers were washed with saturated $\mathrm{NH}_{4} \mathrm{Cl}(1 \times 10 \mathrm{~mL})$ and saturated brine $(1 \times 10 \mathrm{~mL})$, dried over $\mathrm{Na}_{2} \mathrm{SO}_{4}$, filtered, and concentrated. Flash chromatograpy ( $9 \%$ ethyl acetate/hexanes) gave tetra-TBS carbamate (+)-S8 (6.6 mg, 52\%, 73\% based on recovered (+)5) as a colorless oil. $[\square]_{\mathrm{D}}^{23}+51\left(c\right.$ 0.35, $\left.\mathrm{CHCl}_{3}\right)$; IR (film, $\left.\mathrm{NaCl}\right) 3355,2957,2857,1728,1252$, $836 \mathrm{~cm}^{-1} ;{ }^{1} \mathrm{H}$ NMR $\left(500 \mathrm{MHz}, \mathrm{CDCl}_{3}\right) \square 6.59$ (ddd, $\left.J=16.8,10.6,10.6 \mathrm{~Hz}, 1 \mathrm{H}\right), 6.01$ (app t, $J=$ $11.0 \mathrm{~Hz}, 1 \mathrm{H}), 5.82(\mathrm{~m}, 1 \mathrm{H}), 5.38(\operatorname{app~t}, J=10.5 \mathrm{~Hz}, 1 \mathrm{H}), 5.30(\mathrm{~m}, 2 \mathrm{H}), 5.20(\mathrm{~m}, 2 \mathrm{H}), 5.13(\mathrm{~m}$, 2H), $4.80($ app t, $J=8.6 \mathrm{~Hz}, 1 \mathrm{H}), 4.74($ br s, 1H), 4.65 (br s, 1H), 4.51 (app t, $J=10.2 \mathrm{~Hz}, 1 \mathrm{H})$, 3.77 (br s, 2H), 3.65 (app t, $J=2.3 \mathrm{~Hz}, 1 \mathrm{H}$ ), 3.47 (app t, $J=4.4 \mathrm{~Hz}, 1 \mathrm{H}), 3.29$ (app t, $J=5.1 \mathrm{~Hz}$, 1H), $2.98(\mathrm{~m}, 1 \mathrm{H}), 2.64(\mathrm{~m}, 2 \mathrm{H}), 2.54(\mathrm{~m}, 1 \mathrm{H}), 1.90(\mathrm{~m}, 2 \mathrm{H}), 1.90(\mathrm{~m}, 2 \mathrm{H}), 1.81$ (ddd, $J=9.9,6.7$, $2.2 \mathrm{~Hz}, 1 \mathrm{H}), 1.74(\mathrm{~m}, 1 \mathrm{H}), 1.66(\mathrm{~m}, 1 \mathrm{H}), 1.59(\mathrm{ddd}, J=13.6,11.2,1.9 \mathrm{~Hz}, 1 \mathrm{H}), 1.23(\mathrm{~d}, J=7.6$ $\mathrm{Hz}, 3 \mathrm{H}), 0.99(\mathrm{~d}, J=6.8 \mathrm{~Hz}, 3 \mathrm{H}), 0.97(\mathrm{~d}, J=6.7 \mathrm{~Hz}, 6 \mathrm{H}), 0.93(\mathrm{~s}, 9 \mathrm{H}), 0.90(\mathrm{~s}, 9 \mathrm{H}), 0.90(\mathrm{~d}, J=$ $6.4 \mathrm{~Hz}, 6 \mathrm{H}), 0.88(\mathrm{~s}, 9 \mathrm{H}), 0.87(\mathrm{~s}, 9 \mathrm{H}), 0.80(\mathrm{~d}, J=6.7 \mathrm{~Hz}, 3 \mathrm{H}), 0.097(\mathrm{~s}, 3 \mathrm{H}), 0.075(\mathrm{~s}, 3 \mathrm{H})$, $0.072(\mathrm{~s}, 3 \mathrm{H}), 0.065(\mathrm{~s}, 6 \mathrm{H}), 0.058(\mathrm{~s}, 3 \mathrm{H}), 0.049(\mathrm{~s}, 3 \mathrm{H}), 0.020(\mathrm{~s}, 3 \mathrm{H}) ;{ }^{13} \mathrm{C}$ NMR $(125 \mathrm{MHz}$, $\left.\mathrm{CDCl}_{3}\right) \square 173.3,145.1,135.2,134.8,133.7,133.6,132.5,132.1,132.1,129.7,127.2,117.8$ 80.0, 78.6, 77.0, 76.1, 74.9, 64.6, 44.1, 43.4, 42.9, 38.2, 38.1, 37.5, 35.0, 34.5, 34.2, 31.9, 26.3, 
$26.2,25.9,25.7,18.5,18.4,18.1,18.0,17.7,17.1,16.4,16.0,14.5,14.1,10.3,-3.1,-3.5,-3.6,-$ 4.2, -4.3, -4.5, -4.9; high resolution mass spectrum $\left(\mathrm{ES}^{+}\right) \mathrm{m} / \mathrm{z} 1098.7462\left[(\mathrm{M}+\mathrm{Na})^{+}\right.$; calcd for $\left.\mathrm{C}_{59} \mathrm{H}_{113} \mathrm{NO}_{8} \mathrm{NaSi}_{4}: 1098.7441\right]$.

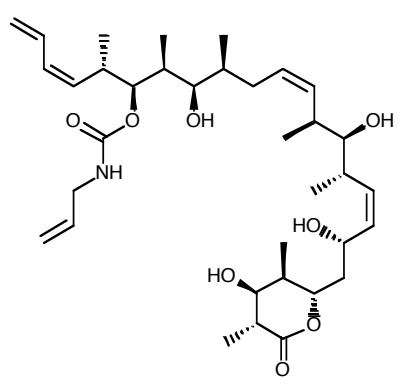

$(+)-14$

Allyl carbamate tetra-ol (+)-14: Tetra-TBS carbamate (+)-S8 (6.0 mg, $5.58 \mu \mathrm{mol})$ was dissolved in $\mathrm{MeOH}(2.0 \mathrm{~mL})$. Aqueous $\mathrm{HCl}(6 \mathrm{~N}, 2.7 \mathrm{~mL})$ was added dropwise over $4 \mathrm{hr}$ at room temperature. The reaction mixture was stirred for $4 \mathrm{hr}$ further, diluted with water $(6 \mathrm{~mL})$ and EtOAc $(10 \mathrm{~mL})$. The resulting mixture was neutralized by solid $\mathrm{NaHCO}_{3}$, and separated. The aqueous layer was extracted with EtOAc $(3 \times 8 \mathrm{~mL})$. The combined organic layers were washed with saturated brine $(1 \times 15 \mathrm{~mL})$, dried over $\mathrm{Na}_{2} \mathrm{SO}_{4}$, filtered, and concentrated. Preparative TLC $\left(9 \% \mathrm{MeOH} / \mathrm{CH}_{2} \mathrm{Cl}_{2}\right)$ provided allyl carbamate tetra-ol (+)-14 (2.2 mg, 64\%) as a colorless solid. $[\square]_{\mathrm{D}}^{23}+36($ c $0.11 \mathrm{MeOH}) ; \mathrm{IR}$ (film, NaCl) 3398, 2967, 1700, 1457, $1245 \mathrm{~cm}^{-1} ;{ }^{1} \mathrm{H}$ NMR (500 $\left.\mathrm{MHz}, \mathrm{CD}_{3} \mathrm{CN}\right) \square 6.68(\mathrm{ddd}, J=16.8,10.5,10.5 \mathrm{~Hz}, 1 \mathrm{H}), 6.05(\operatorname{app} \mathrm{t}, J=11.0 \mathrm{~Hz}, 1 \mathrm{H}), 5.84$ (dddd, $J=17.5,10.1,4.9,4.0 \mathrm{~Hz}, 1 \mathrm{H}$ ), 5.58 (br s, $1 \mathrm{H}$ ), 5.45 (m, 2H), 5.40 (dd, $J=11.0,8.4 \mathrm{~Hz}$, 1H), $5.25(\mathrm{~m}, 3 \mathrm{H}), 5.15(\mathrm{~m}, 2 \mathrm{H}), 5.07(\mathrm{~d}, J=10.3 \mathrm{~Hz}, 1 \mathrm{H}), 4.73($ app t, $J=5.7 \mathrm{~Hz}, 1 \mathrm{H}), 4.54(\mathrm{~m}$, 1H), $4.47(\mathrm{ddd}, J=10.4,10.4,1.9 \mathrm{~Hz}, 1 \mathrm{H}), 3.67(\mathrm{~m}, 3 \mathrm{H}), 3.30(\mathrm{~d}, J=4.9 \mathrm{~Hz}, 1 \mathrm{H}), 3.20(\mathrm{~m}, 1 \mathrm{H})$, $3.15(\mathrm{~m}, 1 \mathrm{H}), 3.07(\mathrm{~m}, 1 \mathrm{H}), 2.83(\mathrm{~d}, J=5.1 \mathrm{~Hz}, 1 \mathrm{H}), 2.66-2.55(\mathrm{~m}, 4 \mathrm{H}), 2.43($ app dq, $J=15.8$, $6.8 \mathrm{~Hz}, 1 \mathrm{H}), 1.91($ app t, $J=4.7 \mathrm{~Hz}, 1 \mathrm{H}), 1.86(\mathrm{ddd}, J=9.9,6.6,3.3 \mathrm{~Hz}, 1 \mathrm{H}), 1.79(\mathrm{~m}, 1 \mathrm{H}), 1.73$ (ddd, $J=14.4,10.3,1.9 \mathrm{~Hz}, 1 \mathrm{H}), 1.73(\mathrm{~m}, 1 \mathrm{H}), 1.62(\mathrm{~m}, 1 \mathrm{H}), 1.56$ (ddd, $J=14.1,10.5,2.5 \mathrm{~Hz}$, 1H), $1.21(\mathrm{~d}, J=7.3 \mathrm{~Hz}, 3 \mathrm{H}), 1.01(\mathrm{~d}, J=6.9 \mathrm{~Hz}, 3 \mathrm{H}), 0.99$ (d, $J=6.9 \mathrm{~Hz}, 3 \mathrm{H}), 0.96(\mathrm{~d}, J=6.8$ 
$\mathrm{Hz}, 3 \mathrm{H}), 0.92(\mathrm{~d}, J=6.7 \mathrm{~Hz}, 3 \mathrm{H}), 0.85(\mathrm{~d}, J=7.1 \mathrm{~Hz}, 3 \mathrm{H}), 0.83(\mathrm{~d}, J=6.7 \mathrm{~Hz}, 3 \mathrm{H}) ;{ }^{13} \mathrm{C}$ NMR $\left(125 \mathrm{MHz}, \mathrm{CD}_{3} \mathrm{CN}\right) \square 174.7,158.2,146.0,136.4,134.9,134.6,134.0,133.6,133.4,130.4$, $128.3,115.1,79.3,79.1,77.9,75.4,73.1,63.8,44.0,43.9,42.3,38.4,35.7,36.6,36.3,35.0$, $32.1,30.2,19.1,18.2,16.6,15.7,15.5,13.1,9.4$; high resolution mass spectrum $\left(E S^{+}\right) \mathrm{m} / \mathrm{z}$ $642.3978\left[(\mathrm{M}+\mathrm{Na})^{+}\right.$; calcd for $\left.\mathrm{C}_{35} \mathrm{H}_{57} \mathrm{NO}_{8} \mathrm{Na}: 642.3981\right]$.

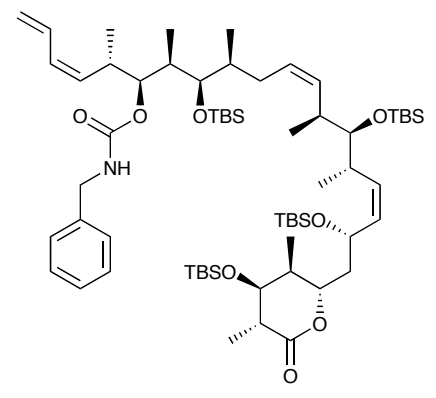

(+)-S9

Benzyl carbamate (+)-S9: A solution of alcohol (+)-5 (13 mg, $0.013 \mathrm{mmol})$ in benzene $(0.80 \mathrm{~mL})$ was treated with benzyl isocyanate $(32 \mu \mathrm{L}, 0.26 \mathrm{mmol})$ and DBU $(28 \mu \mathrm{L}, 0.18 \mathrm{mmol})$ at $45^{\circ} \mathrm{C}$ for $16 \mathrm{hr}$. The reaction mixture was quenched with saturated $\mathrm{NH}_{4} \mathrm{Cl}(2 \mathrm{~mL})$, and diluted with $\mathrm{CH}_{2} \mathrm{Cl}_{2}$ $(5 \mathrm{~mL})$. The resulting layers were separated, and the aqueous layer was extracted with $\mathrm{CH}_{2} \mathrm{Cl}_{2}(3$ x $5 \mathrm{~mL})$. The combined organic layers were washed with saturated brine $(1 \times 8 \mathrm{~mL})$, dried over $\mathrm{Na}_{2} \mathrm{SO}_{4}$, filtered, and concentrated. Flash chromotography (5\% ethyl acetate/ hexanes) provided $11 \mathrm{mg}$ tetra-TBS carbamate $(+)$-S9 $(75 \%)$ as a colorless oil. $[\square]_{\mathrm{D}}^{23}+42.7\left(\mathrm{c} 1.0, \mathrm{CHCl}_{3}\right)$; IR $(\mathrm{NaCl})$ $3356,2957,2929,2884,2859,1725,15.6,1472,1462,1387,1360,1252,1097,1046,836,775$ $\mathrm{cm}^{-1} ;{ }^{1} \mathrm{H}-\mathrm{NMR}\left(500 \mathrm{MHz}, \mathrm{C}_{6} \mathrm{D}_{6}\right) \square 7.24(\mathrm{~m}, 3 \mathrm{H}), 7.17(\mathrm{dd}, J=7.8,6.0,2 \mathrm{H}), 6.76(\mathrm{ddd}, J=16.8$, 10.4, 10.4 Hz, 1H), $6.16(\mathrm{dd}, J=10.8,10.8 \mathrm{~Hz}, 1 \mathrm{H}), 5.71-5.50(\mathrm{~m}, 5 \mathrm{H}), 5.27(\mathrm{~m}, 1 \mathrm{H}), 5.25(\mathrm{dd}, J$ $=16.8,1.9 \mathrm{~Hz}, 1 \mathrm{H}), 5.15(\mathrm{~d}, J=10.4 \mathrm{~Hz}, 1 \mathrm{H}), 5.15(\mathrm{~m}, 1 \mathrm{H}), 4.80$ (ddd, $J=11.2,10.0,1.5 \mathrm{~Hz}$, 1H), $4.42(\mathrm{dd}, J=6.3,6.3 \mathrm{~Hz}, 1 \mathrm{H}), 4.22(\mathrm{dd}, J=6.3,6.3 \mathrm{~Hz}, 1 \mathrm{H}), 4.15(\mathrm{dd}, J=6.3,6.3 \mathrm{~Hz}, 1 \mathrm{H})$, 3.81 (br s, $1 \mathrm{H}$ ), 3.64 (dd, $J=5.2,5.2 \mathrm{~Hz}, 1 \mathrm{H}), 3.51$ (dd, $J=2.6,2.6 \mathrm{~Hz}, 1 \mathrm{H}$ ), 3.14 (ddd, $J=10.0$, 6.3, $6.3 \mathrm{~Hz}, 1 \mathrm{H}), 3.09(\mathrm{~m}, 1 \mathrm{H}), 2.96$ (ddddd, $J=10.0,6.3,6.3,6.3,6.3 \mathrm{~Hz}, 1 \mathrm{H}), 2.76$ (dddd, $J=$ 7.4, 7.4. 7.4, 3.0 Hz, 1H), $2.35(\mathrm{~m}, 2 \mathrm{H}), 2.20-2.18(\mathrm{~m}, 1 \mathrm{H}), 2.18-2.15(\mathrm{~m}, 1 \mathrm{H}), 2.01$ (dddd, $J=$ 
12.6, 10.8, $1.5,1.5 \mathrm{~Hz}, 1 \mathrm{H}), 1.84$ (dddd, $J=11.5,6.7,6.7,1.9 \mathrm{~Hz}, 1 \mathrm{H}), 1.79(\mathrm{~m}, 1 \mathrm{H}), 1.33(\mathrm{~d}, J=$ $7.1 \mathrm{~Hz}, 3 \mathrm{H}), 1.30(\mathrm{~d}, J=6.7 \mathrm{~Hz}, 3 \mathrm{H}), 1.25(\mathrm{br} \mathrm{d}, J=6.7 \mathrm{~Hz}, 3 \mathrm{H}), 1.24(\mathrm{~d}, J=7.5 \mathrm{~Hz}, 3 \mathrm{H}), 1.18$ (s, 9H), 1.18-1.16 (obscured, 3H), $1.17(\mathrm{~s}, 9 \mathrm{H}), 1.14(\mathrm{~s}, 9 \mathrm{H}), 1.07(\mathrm{~d}, J=6.7 \mathrm{~Hz}, 3 \mathrm{H}), 1.02(\mathrm{~s}, 9 \mathrm{H})$, $0.94(\mathrm{~d}, J=6.7 \mathrm{~Hz}, 3 \mathrm{H}), 0.41$ (s, 3H), 0.39 (s, 3H), 0.34 (s, 3H), 0.33 (br s, 3H), $0.32(\mathrm{~s}, 3 \mathrm{H}), 0.29$ (s, 3H), 0.040 (s, 3H), 0.038 (s, 3H); ${ }^{13} \mathrm{C}-\mathrm{NMR}\left(125 \mathrm{MHz}, \mathrm{CDCl}_{3}\right) \square$ 173.1, 156.7, 138.7, 135.1, 133.7, 133.5, 132.4, 132.1, 129.7, 128.5 (2), 127.3, 127.1, 117.7, 79.9, 78.6, 77.1, 76.1, 74.8, $64.6,45.0,44.1,42.8,38.1,38.0,37.5,34.9,34.5,34.1,31.9,26.2,26.1,25.8,25.6,18.5,18.4$, $18.0,17.9,17.5,17.0,16.3,16.0,14.2,14.0,10.2,-3.2,-3.6,-3.7,-4.3,-4.41,-4.6,-4.9$ (2) high resolution mass spectrum $\left(\mathrm{ES}^{+}\right) \mathrm{m} / \mathrm{z} 1148.7616\left[(\mathrm{M}+\mathrm{Na})^{+}\right.$; calcd for $\mathrm{C}_{63} \mathrm{H}_{115} \mathrm{NO}_{8} \mathrm{Si}_{4} \mathrm{Na:}$ 1148.7597]

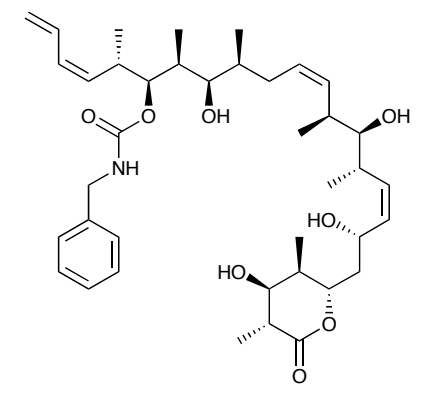

$(+)-15$

Benzyl carbamate tetra-ol (+)-15: Tetra-TBS carbamate (+)-S9 (10 mg, $8.9 \mu \mathrm{mol})$ was dissolved in $\mathrm{MeOH}(2 \mathrm{~mL})$. Aqueous $\mathrm{HCl}(6 \mathrm{~N}, 2.5 \mathrm{~mL})$ was added dropwise over $4 \mathrm{hr}$ at room temperature. The reaction mixture was stirred for $4 \mathrm{hr}$ further, diluted with water $(10 \mathrm{~mL})$ and EtOAc $(15 \mathrm{~mL})$. The resulting mixture was neutralized by solid $\mathrm{NaHCO}_{3}$, and separated. The aqueous layer was extracted with EtOAc $(3 \times 8 \mathrm{~mL})$. The combined organic layers were washed with saturated brine (1 $\times 15 \mathrm{~mL})$, dried over $\mathrm{MgSO}_{4}$, filtered, and concentrated. Preparative TLC $\left(10 \% \mathrm{MeOH} / \mathrm{CH}_{2} \mathrm{Cl}_{2}\right)$ provided benzyl carbamate tetra-ol (+)-15 (4.3 mg, 72\%) as a colorless solid. $[\square]_{D}^{23}+48.3(c 0.33$, $\mathrm{MeOH}$ ); IR (film, NaCl) 3407, 2967, 2925, 2878, 1700, 1518, 1454, 1379, 1248, 1036, $972 \mathrm{~cm}^{-1}$; ${ }^{1} \mathrm{H}$ NMR $\left(500 \mathrm{MHz}, \mathrm{CD}_{3} \mathrm{OD}\right) \mathrm{7}$ 7.33-7.17 (m, 5H), 6.66 (ddd, $\left.J=16.7,10.4,10.4 \mathrm{~Hz}, 1 \mathrm{H}\right), 6.00$ (dd, $J=11.2,10.8 \mathrm{~Hz}, 1 \mathrm{H}), 5.52(\mathrm{dd}, J=10.8,10.8 \mathrm{~Hz}, 1 \mathrm{H}), 5.42(\mathrm{dd}, J=10.8,10.8 \mathrm{~Hz}, 1 \mathrm{H})$, $5.37(\mathrm{dd}, J=10.4,8.9 \mathrm{~Hz}, 1 \mathrm{H}), 5.25-5.17(\mathrm{~m}, 3 \mathrm{H}), 5.11(\mathrm{~d}, J=10.1 \mathrm{~Hz}, 1 \mathrm{H}), 4.83(\mathrm{dd}, J=7.1,4.5$ 
$\mathrm{Hz}, 1 \mathrm{H}), 4.63(\mathrm{dd}, J=9.3,9.3 \mathrm{~Hz}, 1 \mathrm{H}), 4.56(\mathrm{ddd}, J=11.2,9.7,1.1 \mathrm{~Hz}, 1 \mathrm{H}), 4.27(\mathrm{~d}, J=15.6 \mathrm{~Hz}$, 1H), $4.24(\mathrm{~d}, J=15.6 \mathrm{~Hz}, 1 \mathrm{H}), 3.62(\mathrm{dd}, J=4.1,3.7 \mathrm{~Hz}, 1 \mathrm{H}), 3.24(\mathrm{dd}, J=6.7,4.1 \mathrm{~Hz}, 1 \mathrm{H}), 3.16$ (dd, $J=3.7,3.7 \mathrm{~Hz}, 1 \mathrm{H}), 3.15-3.08(\mathrm{~m}, 1 \mathrm{H}), 2.75-2.65(\mathrm{~m}, 1 \mathrm{H}), 2.62$ (dddd, $J=7.4,7.4,7.4,4.5$ $\mathrm{Hz}, 1 \mathrm{H}), 2.45-2.35(\mathrm{~m}, 1 \mathrm{H}), 1.94-1.80(\mathrm{~m}, 3 \mathrm{H}), 1.79-1.55(\mathrm{~m}, 4 \mathrm{H}), 1.24(\mathrm{~d}, J=7.4 \mathrm{~Hz}, 3 \mathrm{H}), 1.04$ (d, $J=7.1 \mathrm{~Hz}, 3 \mathrm{H}), 1.01(\mathrm{~d}, J=6.7 \mathrm{~Hz}, 3 \mathrm{H}), 0.97(\mathrm{~d}, J=7.1 \mathrm{~Hz}, 3 \mathrm{H}), 0.94(\mathrm{~d}, J=6.7 \mathrm{~Hz}, 3 \mathrm{H})$,

$0.90(\mathrm{~d}, J=6.7 \mathrm{~Hz}, 3 \mathrm{H}), 0.86(\mathrm{~d}, J=6.7 \mathrm{~Hz}, 3 \mathrm{H}) ;{ }^{13} \mathrm{C}$ NMR $\left(125 \mathrm{MHz}, \mathrm{CD}_{3} \mathrm{OD}\right) \square$ 175.4, 158.1, $139.4,133.6,132.8,132.7,132.5,132.2,129.5,127.9,127.1,126.6,126.5,116.9,78.8,78.7$, $77.5,74.4,72.1,62.6,43.9,43.0,41.4,37.5,35.7,35.6,35.5,35.2,33.8,31.1,17.8,16.8,15.8$, 14.5, 14.3, 11.8, 8.3; high resolution mass spectrum $\left(\mathrm{ES}^{+}\right) \mathrm{m} / \mathrm{z} 692.4138\left[(\mathrm{M}+\mathrm{Na})^{+}\right.$; calcd for $\mathrm{C}_{39} \mathrm{H}_{59} \mathrm{NO}_{8} \mathrm{Na:}$ 692.4138].

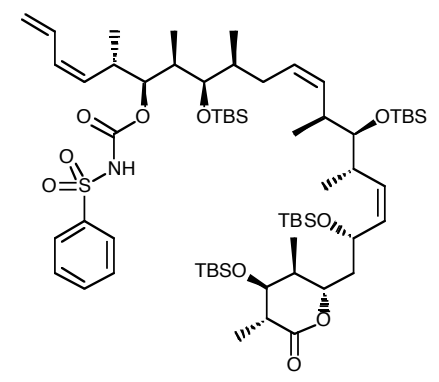

$(+)-\mathbf{S 1 0}$

Benzenesulfonyl carbamate (+)-S10: A solution of alcohol (+)-5 (8.7 mg, $8.77 \mathrm{mmol})$ in $\mathrm{CH}_{2} \mathrm{Cl}_{2}$ $(0.88 \mathrm{~mL})$ was treated with benzenesulfonyl isocyanate $(3.5 \mathrm{~mL}, 26.3 \mathrm{mmol})$ at room temperature for $10 \mathrm{~min}$. The reaction solution was concentrated in vacuo, and purified by flash chromatography (17\% ethyl acetate/hexanes) providing $10.2 \mathrm{mg}$ tetra-TBS carbamate (+)-S10 (99\%) as a colorless oil. $[\square]_{D}^{23}+38\left(c 0.25 \mathrm{CHCl}_{3}\right)$; IR (film, $\left.\mathrm{NaCl}\right) 2929,2857,1746,1462,1253$, 836, $775 \mathrm{~cm}^{-1} ;{ }^{1} \mathrm{H} \mathrm{NMR}\left(500 \mathrm{MHz}, \mathrm{CHCl}_{3}\right) \square 8.02(\mathrm{~d}, J=8.3,2 \mathrm{H}), 7.64(\operatorname{app~t}, J=7.0 \mathrm{~Hz}, 1 \mathrm{H})$, 7.54 (app t, $J=7.7 \mathrm{~Hz}, 2 \mathrm{H}$ ), 7.44 (s, 1H), 6.45 (ddd, $J=16.7,10.7,10.7 \mathrm{~Hz}, 1 \mathrm{H}), 5.74$ (app t, $J=$ $11.0 \mathrm{~Hz}, 1 \mathrm{H}), 5.33(\mathrm{~m}, 2 \mathrm{H}), 5.25(\operatorname{app~t}, J=10.6 \mathrm{~Hz}, 1 \mathrm{H}), 5.13(\mathrm{~m}, 4 \mathrm{H}), 4.81(\operatorname{app~t}, J=9.1 \mathrm{~Hz}$, 1H), $4.70(\operatorname{app~t}, J=6.0 \mathrm{~Hz}, 1 \mathrm{H}), 4.53(\operatorname{app~t}, J=10.4 \mathrm{~Hz}, 1 \mathrm{H}), 3.66(\mathrm{~s}, 1 \mathrm{H}), 3.36(\operatorname{app~t}, J=4.4$ 
$\mathrm{Hz}, 1 \mathrm{H}), 3.29(\operatorname{app~t}, J=5.10 \mathrm{~Hz}, 1 \mathrm{H}), 2.90(\operatorname{app~dq}, J=16.8,6.7 \mathrm{~Hz}, 1 \mathrm{H}), 2.64(\mathrm{~m}, 2 \mathrm{H}), 2.52(\mathrm{~m}$, $1 \mathrm{H}), 1.80(\mathrm{~m}, 5 \mathrm{H}), 1.58(\mathrm{~m}, 2 \mathrm{H}), 1.25(\mathrm{~d}, J=9.4 \mathrm{~Hz}, 3 \mathrm{H}), 1.23(\mathrm{~d}, J=7.8 \mathrm{~Hz}, 3 \mathrm{H}), 0.97(\mathrm{~d}, J=6.6$ $\mathrm{Hz}, 3 \mathrm{H}), 0.97$ (d, J = 6.7 Hz, 3H), $0.91(\mathrm{~s}, 9 \mathrm{H}), 0.89$ (s, 9H), 0.89 (s, 9H), 0.89 (d, J=5.4 Hz, 3H), $0.87(\mathrm{~s}, 9 \mathrm{H}), 0.75$ (d, J = 6.8 Hz, 3H), 0.75 (d, J = 6.7 Hz, 3H), $0.082(\mathrm{~s}, 3 \mathrm{H}), 0.073(\mathrm{~s}, 6 \mathrm{H}), 0.063$ (s, 3H), 0.057 (s, 3H), 0.029 (s, 6H), 0.023 (s, 3H); ${ }^{13} \mathrm{C}$ NMR (125 MHz, $\left.\mathrm{CHCl}_{3}\right) \square$ 173.4, 150.3, $138.8,135.5,133.8,133.6,132.5,132.4,131.6,130.1,128.9,128.2,126.7,118.6,82.0,80.0$ $77.1,76.0,74.8,64.6,44.1,42.9,38.0,37.7,37.6,35.1,34.3,34.2,31.7,29.7,26.3,26.1,25.9$, $25.7,18.5,18.4,18.1,17.9,17.3,17.2,16.4,15.9,14.3,14.1,10.0,-3.1,-3.5,-3.7,-4.2,-4.3,-$ 4.5, -4.9; high resolution mass spectrum $\left(\mathrm{ES}^{+}\right) \quad \mathrm{m} / \mathrm{z} \quad 1198.7059 \quad\left[(\mathrm{M}+\mathrm{Na})^{+}\right.$; calcd for $\left.\mathrm{C}_{62} \mathrm{H}_{113} \mathrm{NO}_{10} \mathrm{NaSi}_{4} \mathrm{~S}: 1198.7060\right]$.

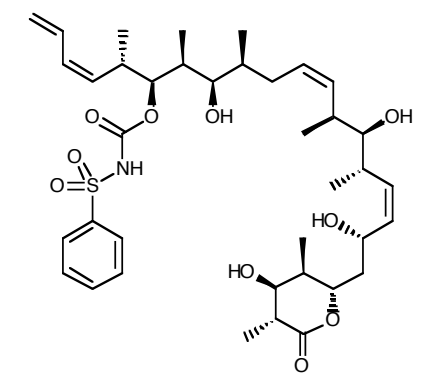

$(+)-16$

Benzenesulfonyl carbamate tetra-ol (+)-16. Tetra-TBS carbamate (+)-S10 (6.0 mg, 0.005 $\mathrm{mmol})$ was dissolved in $\mathrm{MeOH}(2.0 \mathrm{~mL})$ and stirred for $15 \mathrm{~min}$ at room temperature. Aqueous hydrochloric acid $(3 \mathrm{~N}, 5.0 \mathrm{~mL})$ was added in $200-400 \mu \mathrm{L}$ portions over 4 hours at a rate which minimized precipitation (ca. 10 to 15 min intervals). An additional $1 \mathrm{~mL}$ of $3 \mathrm{~N}$ aq HCl was added over $1 \mathrm{~h}$ at $15 \mathrm{~min}$ intervals, and the sides of the flask/stir bar were rinsed with $2.0 \mathrm{~mL}$ of $\mathrm{MeOH}$. After $12 \mathrm{~h}$ the solution was quenched with $\mathrm{NaHCO}_{3}(\mathrm{~s})$, diluted with $30 \mathrm{~mL}$ of water and extracted $3 \times$ with EtOAc. The combined organic extracts were dried $\left(\mathrm{Na}_{2} \mathrm{SO}_{4}\right)$, filtered, and concentrated. Flash chromatography using washed $\mathrm{SiO}_{2}$ (hexanes then $10 \% \mathrm{MeOH} / \mathrm{CH}_{2} \mathrm{Cl}_{2}$ then $5 \%$ $\mathrm{MeOH} / \mathrm{CH}_{2} \mathrm{Cl}_{2}$ ) via gradient elution ( $\left.2 \% \quad \mathrm{MeOH} / \mathrm{CH}_{2} \mathrm{Cl}_{2} \longrightarrow \quad 10 \% \quad \mathrm{MeOH} / \mathrm{CH}_{2} \mathrm{Cl}_{2}\right)$ gave benzenesulfonyl carbamate tetra-ol $(+)-16(3.0 \mathrm{mg}, 83 \%$ yield $) .[\square]_{D}^{23}+65(c 0.08, \mathrm{MeOH})$; IR 
(film, NaCl) 3570, 2968, 1722, 1164, $1090 \mathrm{~cm}^{-1} ;{ }^{1} \mathrm{H}$ NMR $\left(500 \mathrm{MHz}, \mathrm{CD}_{3} \mathrm{CN}\right) \square 7.92$ (d, $J=7.4$ $\mathrm{Hz}, 2 \mathrm{H}), 7.64(\operatorname{app~t}, J=7.4 \mathrm{~Hz}, 1 \mathrm{H}), 7.55(\operatorname{app~t}, J=7.8 \mathrm{~Hz}, 2 \mathrm{H}), 6.58$ (ddd, $J=16.6,10.5,10.5$ $\mathrm{Hz}, 1 \mathrm{H}), 5.92(\operatorname{appt}, J=11.0 \mathrm{~Hz}, 1 \mathrm{H}), 5.47(\operatorname{app~t}, J=10.4 \mathrm{~Hz}, 1 \mathrm{H}), 5.39(\mathrm{dd}, J=11.1,8.2 \mathrm{~Hz}$ 1H), $5.18(\mathrm{~m}, 6 \mathrm{H}), 4.68(\mathrm{dd}, J=7.3,4.7 \mathrm{~Hz}, 1 \mathrm{H}), 4.51(\mathrm{~m}, 1 \mathrm{H}), 4.44(\mathrm{~m}, 1 \mathrm{H}), 3.67(\operatorname{app~t}, J=4.7$ $\mathrm{Hz}, 1 \mathrm{H}), 3.14(\mathrm{~m}, 1 \mathrm{H}), 3.10(\mathrm{dd}, J=7.2,4.1 \mathrm{~Hz}, 1 \mathrm{H}), 3.00(\mathrm{app} \mathrm{dq}, J=14.0,7.2 \mathrm{~Hz}, 1 \mathrm{H}), 2.61(\mathrm{~m}$, 3H), $2.38($ app dq, $J=16.2,6.8 \mathrm{~Hz}, 1 \mathrm{H}), 1.86(\mathrm{~m}, 3 \mathrm{H}), 1.72(\mathrm{~m}, 3 \mathrm{H}), 1.58(\mathrm{~m}, 3 \mathrm{H}), 1.47(\mathrm{~m}, 1 \mathrm{H})$, $1.19(\mathrm{~d}, J=7.3 \mathrm{~Hz}, 3 \mathrm{H}), 1.01$ (d, $J=6.9 \mathrm{~Hz}, 3 \mathrm{H}), 0.99$ (d, $J=6.9 \mathrm{~Hz}, 3 \mathrm{H}), 0.90(\mathrm{~d}, J=6.7 \mathrm{~Hz}$, $3 \mathrm{H}), 0.82(\mathrm{~d}, J=6.8 \mathrm{~Hz}, 3 \mathrm{H}), 0.78(\mathrm{~d}, J=6.6 \mathrm{~Hz}, 3 \mathrm{H}), 0.66(\mathrm{~d}, J=6.8 \mathrm{~Hz}, 3 \mathrm{H}) ;{ }^{13} \mathrm{C}$ NMR $(125$ $\left.\mathrm{MHz}, \mathrm{CD}_{3} \mathrm{CN}\right) \square 174.9,156.8,141.7,134.9,134.0(2), 133.9,133.8,133.7,133.1,130.6,129.8$, 128.3, 118.6, 81.8, 79.3, 78.1, 75.1, 72.7, 63.8, 43.6, 42.5, 38.1, 36.8, 36.6, 36.5, 35.4, 34.6, $31.9,19.2,17.9,16.7,15.6,15.4,13.0,9.2$; high resolution mass spectrum (ES $\left.{ }^{+}\right) \mathrm{m} / \mathrm{z} 742.3635$ $\left[(\mathrm{M}+\mathrm{Na})^{+}\right.$; calcd for $\left.\mathrm{C}_{38} \mathrm{H}_{57} \mathrm{NO}_{10} \mathrm{NaS}: 742.3601\right]$.

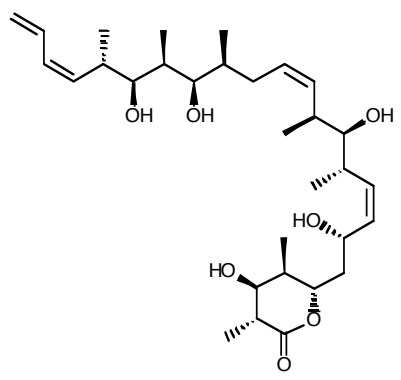

$(+)-17$

Penta-ol (+)-17: Tetra-TBS alcohol (+)-5 (6.0 mg, $0.006 \mathrm{mmol})$ was dissolved in $\mathrm{MeOH}(1.0 \mathrm{~mL})$ and stirred for $15 \mathrm{~min}$ at room temperature. Aqueous hydrochloric acid $(3 \mathrm{~N}, 5.0 \mathrm{~mL})$ was added in 200-400 $\mu \mathrm{L}$ portions over 4 hours at a rate which minimized precipitation (ca. 10 to $15 \mathrm{~min}$ intervals). An additional $2 \mathrm{~mL}$ of $3 \mathrm{~N}$ aq HCl was added over $1 \mathrm{~h}$ at $15 \mathrm{~min}$ intervals, and the sides of the flask/stir bar were rinsed with $2.0 \mathrm{~mL}$ of $\mathrm{MeOH}$. After $12 \mathrm{~h}$ the solution was quenched with $\mathrm{NaHCO}_{3}(\mathrm{~s})$, diluted with $30 \mathrm{~mL}$ of water and extracted $3 \times$ with EtOAc. The combined organic extracts were dried $\left(\mathrm{Na}_{2} \mathrm{SO}_{4}\right)$, filtered, and concentrated. Flash chromatography using 
washed $\mathrm{SiO}_{2}$ (hexanes then $10 \% \mathrm{MeOH} / \mathrm{CH}_{2} \mathrm{Cl}_{2}$ then $5 \% \mathrm{MeOH} / \mathrm{CH}_{2} \mathrm{Cl}_{2}$ ) via gradient elution (5\% MeOH/CH $\left.\mathrm{Cl}_{2} \longrightarrow 10 \% \mathrm{MeOH} / \mathrm{CH}_{2} \mathrm{Cl}_{2}\right)$ gave penta-ol (+)-17 (2.7 mg, 84\% yield). $[\square]_{\mathrm{D}}^{23}+44$ (c $0.135 \mathrm{MeOH})$; IR (film, NaCl) 3420, 2968, 1717, 1457, 1102, $970 \mathrm{~cm}^{-1} ;{ }^{1} \mathrm{H}$ NMR $(500 \mathrm{MHz}$, $\mathrm{CD}_{3} \mathrm{CN}$ ) $\square 6.68$ (ddd, $\left.J=16.9,10.6,10.6 \mathrm{~Hz}, 1 \mathrm{H}\right), 6.06$ (app t, $\left.J=11.0 \mathrm{~Hz}, 1 \mathrm{H}\right), 5.41(\mathrm{~m}, 2 \mathrm{H})$, $5.29(\mathrm{~m}, 2 \mathrm{H}), 5.20(\mathrm{dd}, J=16.9,1.9 \mathrm{~Hz}, 1 \mathrm{H}), 5.10(\mathrm{~d}, J=10.2 \mathrm{~Hz}, 1 \mathrm{H}), 4.52(\operatorname{app~t}, J=9.0 \mathrm{~Hz}$ 1H), 4.46 (ddd, $J=10.3,10.3,1.9 \mathrm{~Hz}, 1 \mathrm{H}), 3.63(\operatorname{app} t, J=3.8 \mathrm{~Hz}, 1 \mathrm{H}), 3.40(\mathrm{~m}, 2 \mathrm{H}), 3.32$ (ddd, $J$ $=7.2,3.6,3.6 \mathrm{~Hz}, 1 \mathrm{H}), 3.28(\mathrm{br} \mathrm{s}, 1 \mathrm{H}), 3.14(\mathrm{dd}, J=11.1,5.1 \mathrm{~Hz}, 1 \mathrm{H}), 3.00(\mathrm{~d}, J=4.3 \mathrm{~Hz}, 1 \mathrm{H})$, $2.89(\mathrm{~d}, J=4.0 \mathrm{~Hz}, 1 \mathrm{H}), 2.82(\mathrm{~m}, 2 \mathrm{H}), 2.64(\mathrm{~m}, 2 \mathrm{H}), 2.56$ (ddd, $J=14.6,7.4,4.6 \mathrm{~Hz}, 1 \mathrm{H}), 2.45$ (m, 1H), $1.98(\mathrm{~m}, 1 \mathrm{H}), 1.98(\mathrm{ddd}, J=14.0,4.4,4.4 \mathrm{~Hz}, 1 \mathrm{H}), 1.68-1.88(\mathrm{~m}, 3 \mathrm{H}), 1.58(\mathrm{~m}, 2 \mathrm{H}), 1.20$ (d, $J=7.3 \mathrm{~Hz}, 3 \mathrm{H}), 1.00(\mathrm{~d}, J=6.8 \mathrm{~Hz}, 3 \mathrm{H}), 0.97(\mathrm{~d}, J=7.0 \mathrm{~Hz}, 3 \mathrm{H}), 0.92(\mathrm{~d}, J=6.7 \mathrm{~Hz}, 3 \mathrm{H})$, $0.91(\mathrm{~d}, J=6.8 \mathrm{~Hz}, 3 \mathrm{H}), 0.88(\mathrm{~d}, J=6.9 \mathrm{~Hz}, 3 \mathrm{H}), 0.85(\mathrm{~d}, J=6.6 \mathrm{~Hz}, 3 \mathrm{H}) ;{ }^{13} \mathrm{C}$ NMR $(125 \mathrm{MHz}$, $\left.\mathrm{CD}_{3} \mathrm{CN}\right) \square 174.7,136.8,135.1,134.1,133.8,133.7,130.4,128.2,117.9,79.3,79.2,79.1,78.0$ 73.2, 63.9, 44.0, 42.4, 37.3, 37.0, 36.7, 36.7, 36.2, 32.1, 30.6, 19.0, 18.0, 16.5, 15.8, 15.7, 13.1, 7.2; high resolution mass spectrum $\left(\mathrm{ES}^{+}\right) \mathrm{m} / z$ 559.3610 $\left[(\mathrm{M}+\mathrm{Na})^{+}\right.$; calcd for $\mathrm{C}_{31} \mathrm{H}_{52} \mathrm{O}_{7} \mathrm{Na}$ : $559.3611]$.

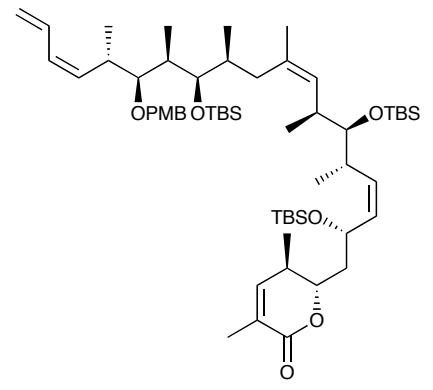

(+)-S11

Tetraene (+)-S11. Phosphonium salt (+)-AB (1.1 g, $0.84 \mathrm{mmol} ; 10: 1$ ratio of diene isomers), was azeotropically dried with benzene $(3 \times 0.5 \mathrm{~mL})$ using a double manifold and further dried by heating to $50{ }^{\circ} \mathrm{C}$ under vacuum ( 0.2 torr) for $16 \mathrm{~h}$. The flask was back-filled with argon, the salt dissolved in $4.5 \mathrm{~mL}$ of freshly distilled THF, and cooled to $-24{ }^{\circ} \mathrm{C}$. The resultant solution was 
treated with methyllithium-lithium bromide complex $(2.2 \mathrm{M}$ in pentane, $0.382 \mathrm{~mL})$, warmed to $0{ }^{\circ} \mathrm{C}$, stirred for 30 min., and then recooled to $-24{ }^{\circ} \mathrm{C}$. To this orange/red solution was transferred dropwise via syringe a solution of aldehyde (-)-18 (below) $(222 \mathrm{mg}, 0.71 \mathrm{mmol})$ in THF $(1.0 \mathrm{~mL}+$ $0.5 \mathrm{~mL}$ rinse) over $30 \mathrm{~min}$. The resulting orange solution was stirred for a further $3 \mathrm{~h}$. at $-24{ }^{\circ} \mathrm{C}$, and then warmed to $-10{ }^{\circ} \mathrm{C}$ and allowed to stir for $1 \mathrm{~h}$. The resulting light yellow solution was quenched with saturated $\mathrm{NH}_{4} \mathrm{Cl}$ and diluted $\left(\mathrm{Et}_{2} \mathrm{O} / \mathrm{H}_{2} \mathrm{O}\right)$. The layers were separated, and the aqueous layer was extracted $\left(3 \times \mathrm{Et}_{2} \mathrm{O}\right)$. The combined organic layers were dried $\left(\mathrm{MgSO}_{4}\right)$ and concentrated. Flash chromatography (gradient elution: 2\% EtOAc/hexanes $\quad 50 \%$ EtOAc/hexanes; then $10 \% \mathrm{MeOH} / \mathrm{CH}_{2} \mathrm{Cl}_{2}$ ) to afford cis isomer (+)-S11 (492 mg, 65\%; clear oil). $[\square]_{D}^{23}+42.5\left(c 0.2 \mathrm{CHCl}_{3}\right) ; \mathrm{IR}(\mathrm{NaCl}) \quad 2954,2929,2858,1727,1612,1511,1462,1377,1254$, 1065, 833, $775 \mathrm{~cm}^{-1} ;{ }^{1} \mathrm{H}-\mathrm{NMR}\left(500 \mathrm{MHz}, \mathrm{CDCl}_{3}\right) \square 7.27(\mathrm{~d}, J=8.6 \mathrm{~Hz}, 2 \mathrm{H}), 6.87(\mathrm{~d}, J=8.6 \mathrm{~Hz}$, 2H), 6.59 (ddd, $J=16.8,10.4,10.4 \mathrm{~Hz}, 1 \mathrm{H}$ ), 6.25 (br s, $1 \mathrm{H}$ ), 6.02 (dd, $J=10.8,10.8 \mathrm{~Hz}, 1 \mathrm{H}$ ), 5.56 (dd, $J=10.4,10.4 \mathrm{~Hz}, 1 \mathrm{H}), 5.28-5.19(\mathrm{~m}, 3 \mathrm{H}), 5.12(\mathrm{~d}, J=10.4 \mathrm{~Hz}, 1 \mathrm{H}), 5.00(\mathrm{~d}, J=10.0 \mathrm{~Hz}$, 1H), 4.77-4.72 (m, 1H), $4.56(\mathrm{~d}, J=10.4 \mathrm{~Hz}, 1 \mathrm{H}), 4.47$ (d, $J=10.4 \mathrm{~Hz}, 1 \mathrm{H}), 4.19$ (ddd, $J=10.0$, 10.0, $1.9 \mathrm{~Hz}, 1 \mathrm{H}), 3.80(\mathrm{~s}, 3 \mathrm{H}), 3.43(\mathrm{dd}, J=5.2,3.4 \mathrm{~Hz}, 1 \mathrm{H}), 3.28-3.22(\mathrm{~m}, 2 \mathrm{H}), 3.00$ (dddd, $J=$ 10.2, 10.2, 6.7, $6.7 \mathrm{~Hz}, 1 \mathrm{H}), 2.64-2.59(\mathrm{~m}, 1 \mathrm{H}), 2.45-2.32(\mathrm{~m}, 2 \mathrm{H}), 1.98(\mathrm{dd}, J=12.4,12.4 \mathrm{~Hz}$, 1H), $1.89(\mathrm{~s}, 3 \mathrm{H}), 1.85-1.55(\mathrm{~m}, 5 \mathrm{H}), 1.55(\mathrm{~s}, 3 \mathrm{H}), 1.12(\mathrm{~d}, J=6.7 \mathrm{~Hz}, 3 \mathrm{H}), 1.02(\mathrm{~d}, J=7.1 \mathrm{~Hz}$, 3H), 0.99 (d, $J=6.7 \mathrm{~Hz}, 3 \mathrm{H}), 0.97(\mathrm{~d}, J=7.1 \mathrm{~Hz}, 3 \mathrm{H}), 0.95(\mathrm{~s}, 9 \mathrm{H}), 0.92(\mathrm{~s}, 9 \mathrm{H}), 0.87$ (d, $J=7.1$ $\mathrm{Hz}, 3 \mathrm{H}), 0.86(\mathrm{~s}, 9 \mathrm{H}), 0.72(\mathrm{~d}, J=6.7 \mathrm{~Hz}, 3 \mathrm{H}), 0.11(\mathrm{~s}, 3 \mathrm{H}), 0.10(\mathrm{~s}, 3 \mathrm{H}), 0.09(\mathrm{~s}, 3 \mathrm{H}), 0.057$ (s, 3H), 0.049 (s, 3H), 0.036 (s, 3H); ${ }^{13} \mathrm{C}-\mathrm{NMR}\left(125 \mathrm{MHz}, \mathrm{CDCl}_{3}\right) \square 165.2,158.9,145.7,134.2,132.9$, $132.4,132.0,131.6,131.5,131.1,129.1,129.0,128.7,127.1,117.6,113.6,84.5,80.6,79.7$, $77.0,75.0,64.5,55.2,41.8,40.1,37.3,35.9,35.4$, 35.2 (2), 33.9, 26.24, 26.18, 25.8, 22.9, 18.60, $18.57,18.4,18.0,17.3,16.93,16.85,16.6,15.0,10.3,-3.1,-3.2,-3.3,-4.3(2),-4.9$; high resolution mass spectrum (ES+) $\mathrm{m} / \mathrm{z} 1017.6880\left[(\mathrm{M}+\mathrm{Na})^{+}\right.$; calcd for $\left.\mathrm{C}_{58} \mathrm{H}_{102} \mathrm{O}_{7} \mathrm{Si}_{3} \mathrm{Na}: 1017.6831\right]$. 


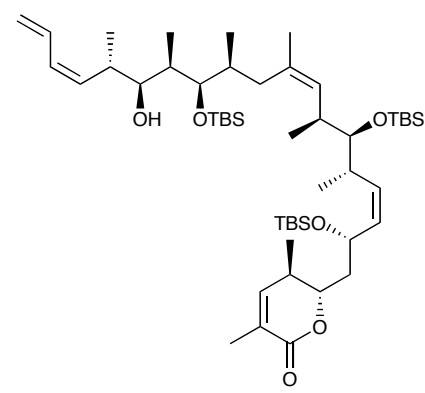

$(+)-19$

Alcohol (+)-19. At $0{ }^{\circ} \mathrm{C}$, a solution of PMB ether (+)-S11 (472 mg, $0.468 \mathrm{mmol}, 10: 1$ mixture of cis/trans diene isomers) in $\mathrm{CH}_{2} \mathrm{Cl}_{2}(7 \mathrm{~mL})$ was treated with $\mathrm{H}_{2} \mathrm{O}(250 \mathrm{LL})$ and $\mathrm{DDQ}(138 \mathrm{mg}$, $0.607 \mathrm{mmol}$ ). The mixture was stirred for $10 \mathrm{~min}$. at $0{ }^{\circ} \mathrm{C}$, warmed to $\mathrm{rt}$ and stirred an additional $20 \mathrm{~min}$. The mixture was quenched with $27.0 \mathrm{~mL}$ saturated $\mathrm{NaHCO}_{3}$, and diluted with $\mathrm{CH}_{2} \mathrm{Cl}_{2}$ (150 mL) and washed with $\mathrm{H}_{2} \mathrm{O}(250 \mathrm{~mL})$ and brine $(250 \mathrm{~mL})$. The layers were separated and the organic layer was dried $\left(\mathrm{MgSO}_{4}\right)$, filtered, and concentrated. Flash chromatography $(5 \%$ EtOAc) provided (+)-19 (358 mg, 86\%) as a colorless oil: $[\square]_{\mathrm{D}}^{23}+5.0\left(\right.$ c $\left.0.1 \mathrm{CHCl}_{3}\right) ; \mathrm{IR}(\mathrm{NaCl})$ $3444,2951,2920,2857,1722,1455,1371,1251,1093,1067,1041,832,775 \mathrm{~cm}^{-1} ;{ }^{1} \mathrm{H}-\mathrm{NMR}$ $\left(500 \mathrm{MHz}, \mathrm{CDCl}_{3}\right) \square 6.63(\mathrm{ddd}, J=16.8,10.4,10.4 \mathrm{~Hz}, 1 \mathrm{H}), 6.31(\mathrm{dd}, J=2.2,1.5 \mathrm{~Hz}, 1 \mathrm{H}), 6.14$ (dd, $J=10.8,10.8 \mathrm{~Hz}, 1 \mathrm{H}), 5.34(\mathrm{dd}, J=10.4,10.4 \mathrm{~Hz}, 1 \mathrm{H}), 5.27-5.22(\mathrm{~m}, 3 \mathrm{H}), 5.15(\mathrm{~d}, J=10.4$ $\mathrm{Hz}, 1 \mathrm{H}$ ), 5.06 (d, $J=10.4 \mathrm{~Hz}, 1 \mathrm{H}$ ), 4.76 (ddd, $J=10.0,6.7,3.4 \mathrm{~Hz}, 1 \mathrm{H}$ ), 4.20 (ddd, $J=10.0,10.0$, $3.0 \mathrm{~Hz}, 1 \mathrm{H}), 3.62(\mathrm{dd}, J=5.6,3.3 \mathrm{~Hz}, 1 \mathrm{H}), 3.36-3.32(\mathrm{~m}, 1 \mathrm{H}), 3.27(\mathrm{dd}, J=5.2,5.2 \mathrm{~Hz}, 1 \mathrm{H}), 2.81$ (dddd, $J=16.8,7.1,7.1,7.1 \mathrm{~Hz}, 1 \mathrm{H}), 2.65-2.58(\mathrm{~m}, 1 \mathrm{H}), 2.52-2.44(\mathrm{~m}, 1 \mathrm{H}), 2.42-2.33(\mathrm{~m}, 1 \mathrm{H})$, $2.18(\mathrm{dd}, J=12.2,12.2 \mathrm{~Hz}, 1 \mathrm{H}), 1.90(\mathrm{dd}, J=1.5,1.5 \mathrm{~Hz}, 3 \mathrm{H}), 1.89-1.84(\mathrm{~m}, 1 \mathrm{H}), 1.84-1.79(\mathrm{~m}$, 1H), 1.77-1.66 (m, 3H), $1.59(\mathrm{~s}, 3 \mathrm{H}), 1.07$ (d, J = 6.7 Hz, 3H), 0.98 (d, J=6.7 Hz, 3H), 0.975 (d, J $=6.7 \mathrm{~Hz}, 3 \mathrm{H}), 0.96(\mathrm{~d}, J=7.1 \mathrm{~Hz}, 3 \mathrm{H}), 0.93(\mathrm{~s}, 9 \mathrm{H}), 0.91(\mathrm{~s}, 9 \mathrm{H}), 0.90(\mathrm{~d}, J=6.7 \mathrm{~Hz}, 3 \mathrm{H}), 0.86(\mathrm{~s}$, 9H), $0.74(\mathrm{~d}, J=6.7 \mathrm{~Hz}, 3 \mathrm{H}), 0.09(\operatorname{app~s}, 6 \mathrm{H}), 0.08(\mathrm{~s}, 3 \mathrm{H}), 0.06(\mathrm{~s}, 3 \mathrm{H}), 0.04(\mathrm{~s}, 3 \mathrm{H}), 0.03$ (s, $3 \mathrm{H}) ;{ }^{13} \mathrm{C}-\mathrm{NMR}\left(125 \mathrm{MHz}, \mathrm{CDCl}_{3}\right) \square 165.2,145.6,134.6,133.0,132.5,132.0,131.7,131.6,130.9$, $127.2,118.3,80.5,79.7,78.7,76.3,64.6,41.8,40.0,37.3,36.2,36.1,35.4,35.0,33.9,26.2$, $26.1,25.8,23.1,18.40,18.37,18.0,17.3,17.2,16.8,16.6,16.5,13.6,9.4,-3.1,-3.4,-3.7,-4.33,-$ 
4.34, -4.9; high resolution mass spectrum (ES+) $\mathrm{m} / \mathrm{z} 897.6248\left[(\mathrm{M}+\mathrm{Na})^{+}\right.$; calcd for $\left.\mathrm{C}_{50} \mathrm{H}_{94} \mathrm{O}_{6} \mathrm{Si}_{3} \mathrm{Na}: 897.6256\right]$.

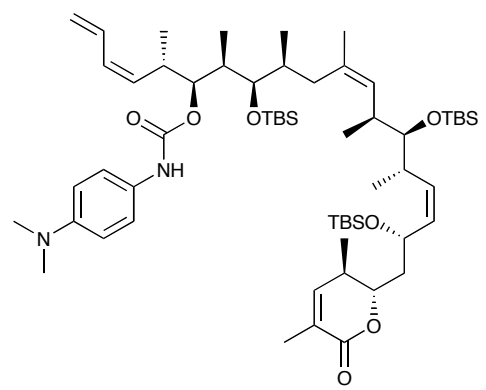

(+)-S12

Dimethylaniline carbamate (+)-S12: A solution of alcohol (+)-19 (13 mg, $0.015 \mathrm{mmol})$ in pyridine $(1.0 \mathrm{~mL})$ was treated with 4-dimethylaminophenyl isocyanate $(60 \mathrm{mg}, 0.37 \mathrm{mmol})$ at room temperature for $24 \mathrm{hr}$. The reaction mixture was quenched with saturated $\mathrm{NH}_{4} \mathrm{Cl}(2 \mathrm{~mL})$, and diluted with $\mathrm{CH}_{2} \mathrm{Cl}_{2}(5 \mathrm{~mL})$. The resulting layers were separated, and the aqueous layer was extracted with $\mathrm{CH}_{2} \mathrm{Cl}_{2}(3 \times 5 \mathrm{~mL})$. The combined organic layers were washed with saturated brine $(1 \times 8 \mathrm{~mL})$, dried over $\mathrm{Na}_{2} \mathrm{SO}_{4}$, filtered, and concentrated. Flash chromotography (5\% to $10 \%$ ethyl acetate/ hexanes) provided $14.5 \mathrm{mg}$ tri-TBS carbamate (+)-S12 (95\%) as a colorless oil. $[\square]_{\mathrm{D}}^{23}+41.9$ (c 1.0, $\left.\mathrm{CHCl}_{3}\right)$; IR (NaCl) 3340, 2957, 2930, 2883, 2849, 1725, 1525, 1472, 1367, 1218, 1134, 1099, 1043, 948, 835, $774 \mathrm{~cm}^{-1} ;{ }^{1} \mathrm{H}-\mathrm{NMR}\left(500 \mathrm{MHz}, \mathrm{CDCl}_{3}\right) \square 7.20$ (app br s, 2H), $6.69(\operatorname{app~d}, J=7.1 \mathrm{~Hz}, 2 \mathrm{H}), 6.63(\mathrm{~m}, 1 \mathrm{H}), 6.31(\mathrm{br} \mathrm{s}, 1 \mathrm{H}), 6.23(\mathrm{~s}, 1 \mathrm{H}), 6.02(\mathrm{dd}, J=10.8,10.8$ $\mathrm{Hz}, 1 \mathrm{H}), 5.39(\mathrm{dd}, J=10.4,10.4 \mathrm{~Hz}, 1 \mathrm{H}), 5.29-5.22(\mathrm{~m}, 2 \mathrm{H}), 5.21(\mathrm{~d}, J=16.8 \mathrm{~Hz}, 1 \mathrm{H}), 5.14(\mathrm{~d}, J$ $=10.0 \mathrm{~Hz}, 1 \mathrm{H}), 5.03(\mathrm{~d}, J=10.0 \mathrm{~Hz}, 1 \mathrm{H}), 4.83-4.78(\mathrm{~m}, 1 \mathrm{H}), 4.77-4.73(\mathrm{~m}, 1 \mathrm{H}), 4.18(\mathrm{ddd}, J=$ 10.0, 10.0, 3.0 Hz, 1H), 3.48 (dd, J=4.1, $4.1 \mathrm{~Hz}, 1 \mathrm{H}$ ), 3.27 (dd, $J=5.2,5.2 \mathrm{~Hz}, 1 \mathrm{H}$ ), 3.03 (ddd, $J=6.7,6.7,6.7,3.4 \mathrm{~Hz}, 1 \mathrm{H}), 2.90(\mathrm{~s}, 6 \mathrm{H}), 2.63-2.56(\mathrm{~m}, 1 \mathrm{H}), 2.50-2.42(\mathrm{~m}, 1 \mathrm{H}), 2.37-2.30(\mathrm{~m}$, 1H), 2.14 (dd, $J=12.3,12.3 \mathrm{~Hz}, 1 \mathrm{H}), 1.98-1.87(\mathrm{~m}, 2 \mathrm{H}), 1.88(\mathrm{dd}, J=2.2,1.5 \mathrm{~Hz}, 3 \mathrm{H}), 1.74-1.62$ (m, 3H), $1.59(\mathrm{~s}, 3 \mathrm{H}), 1.03(\mathrm{~d}, J=6.7 \mathrm{~Hz}, 3 \mathrm{H}), 1.02(\mathrm{~d}, J=7.1 \mathrm{~Hz}, 3 \mathrm{H}), 0.98(\mathrm{~d}, J=7.1 \mathrm{~Hz}, 3 \mathrm{H})$, $0.94(\mathrm{br} \mathrm{d}, J=6.7 \mathrm{~Hz}, 3 \mathrm{H}), 0.94(\mathrm{~s}, 9 \mathrm{H}), 0.91(\mathrm{~s}, 9 \mathrm{H}), 0.90(\mathrm{~d}, J=6.7 \mathrm{~Hz}, 3 \mathrm{H}), 0.86(\mathrm{~s}, 9 \mathrm{H}), 0.74$ 
(d, $J=6.7 \mathrm{~Hz}, 3 \mathrm{H}), 0.11(\mathrm{~s}, 3 \mathrm{H}), 0.09(\mathrm{~s}, 3 \mathrm{H}), 0.08(\mathrm{~s}, 3 \mathrm{H}), 0.05(\mathrm{~s}, 3 \mathrm{H}), 0.045(\mathrm{~s}, 3 \mathrm{H}), 0.038(\mathrm{~s}$, $3 \mathrm{H}) ;{ }^{13} \mathrm{C}-\mathrm{NMR}\left(125 \mathrm{MHz}, \mathrm{CDCl}_{3}\right) \square$ 165.2, 154.0, 145.7, 133.7, 133.0, 132.3, 132.1, 131.6, 131.5, 129.8, 127.1, 120.7 (br), 117.9, 113.4 (br), (2 quaternary aromatic signals are significantly broadened and could not be resolved), 80.5, 79.7, 78.6 (br), 77.0, 64.5, 41.6, 41.02, 37.7, 37.3, $36.1,35.5,35.1,34.5,33.9,26.20,26.15,25.8,22.9,18.5,18.4,18.0,17.5,17.3,16.84,16.82$, $16.5,13.5,10.2,-3.1,-3.4,-3.5,-4.30,-4.35,4.9$; high resolution mass spectrum $\left(\mathrm{ES}^{+}\right) \mathrm{m} / \mathrm{z}$ 1059.7024 [(M+Na) $)^{+}$; calcd for $\mathrm{C}_{59} \mathrm{H}_{104} \mathrm{~N}_{2} \mathrm{O}_{7} \mathrm{Si}_{3} \mathrm{Na}:$ 1059.7049]

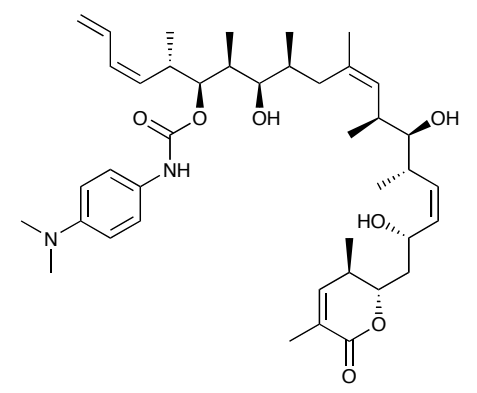

$(+)-21$

Dimethylaniline carbamate triol (+)-21: Tri-TBS carbamate (+)-S12 (5.0 mg, $4.8 \mu \mathrm{mol})$ was dissolved in $\mathrm{MeOH}(1 \mathrm{~mL})$. Aqueous $\mathrm{HCl}(4 \mathrm{~N}, 4 \mathrm{~mL})$ was added dropwise over $2 \mathrm{hr}$ at room temperature. The reaction mixture was stirred for $4 \mathrm{hr}$ further, diluted with water $(10 \mathrm{~mL})$ and EtOAc $(25 \mathrm{~mL})$. The resulting mixture was neutralized by solid $\mathrm{NaHCO}_{3}$, and separated. The aqueous layer was extracted with EtOAc $(3 \times 8 \mathrm{~mL})$. The combined organic layers were washed with saturated brine $(1 \times 15 \mathrm{~mL})$, dried over $\mathrm{Na}_{2} \mathrm{SO}_{4}$, filtered, and concentrated. Flash chromotography ( $2 \%$ to $5 \% \mathrm{MeOH} / \mathrm{CH}_{2} \mathrm{Cl}_{2}$ ) provided dimethylaniline carbamate triol (+)-21 (3.3 mg, 96\%) as a colorless solid. $[\square]_{D}^{23}+36.0(c$ 0.33, MeOH); IR (film, $\mathrm{NaCl}) 3446,2965,2929$, $2872,1699,1525,1456,1374,1320,1225,1141,1084,1045 \mathrm{~cm}^{-1} ;{ }^{1} \mathrm{H}$ NMR $\left(500 \mathrm{MHz}, \mathrm{CD}_{3} \mathrm{OD}\right)$ $\square 7.23(\mathrm{br} \mathrm{d}, J=5.6 \mathrm{~Hz}, 2 \mathrm{H}), 6.80-6.70(\mathrm{~m}, 2 \mathrm{H}), 6.70(\mathrm{ddd}, J=16.7,10.4,10.4 \mathrm{~Hz}, 1 \mathrm{H}), 6.32$ (ddd, $J=2.6,1.5,1.5 \mathrm{~Hz}, 1 \mathrm{H}$ ), 6.07 (dd, $J=11.2,10.8 \mathrm{~Hz}, 1 \mathrm{H}), 5.62$ (ddd, $J=10.8,9.7,1.1 \mathrm{~Hz}$, $1 \mathrm{H}), 5.48(\mathrm{dd}, J=11.5,10.4 \mathrm{~Hz}, 1 \mathrm{H}), 5.38(\mathrm{dd}, J=11.2,8.9 \mathrm{~Hz}, 1 \mathrm{H}), 5.26$ (dd, $J=16.8,1.9 \mathrm{~Hz}$, $1 \mathrm{H}), 5.16(\mathrm{~d}, J=10.4 \mathrm{~Hz}, 1 \mathrm{H}), 4.97(\mathrm{~d}, J=10.1 \mathrm{~Hz}, 1 \mathrm{H}), 4.94-4.88(\mathrm{~m}, 1 \mathrm{H}), 4.82-4.75(\mathrm{~m}, 1 \mathrm{H})$, 
4.59 (dd, $J=9.3,9.3 \mathrm{~Hz}, 1 \mathrm{H}), 4.25$ (ddd, $J=10.4,10.4,1.9 \mathrm{~Hz}, 1 \mathrm{H}), 3.25$ (dd, $J=6.7,4.8 \mathrm{~Hz}$, 1H), 3.21-3.13 (m, 1H), $3.13(\mathrm{dd}, J=8.6,3.0 \mathrm{~Hz}, 1 \mathrm{H}), 2.88(\mathrm{br} \mathrm{s}, 6 \mathrm{H}), 2.75-2.66(\mathrm{~m}, 1 \mathrm{H}), 2.42-$ $2.30(\mathrm{~m}, 2 \mathrm{H}), 1.97(\mathrm{dd}, J=11.9,11.9 \mathrm{~Hz}, 1 \mathrm{H}), 1.91-1.83(\mathrm{~m}, 2 \mathrm{H}), 1.81(\mathrm{dd}, J=2.2,1.5 \mathrm{~Hz}, 3 \mathrm{H})$, $1.75(\mathrm{dd}, J=12.3,12.3 \mathrm{~Hz}, 1 \mathrm{H}), 1.71-1.63(\mathrm{~m}, 2 \mathrm{H}), 1.63(\mathrm{~s}, 3 \mathrm{H}), 1.08(\mathrm{~d}, J=7.1 \mathrm{~Hz}, 3 \mathrm{H}), 1.03$ (app d, $J=6.7 \mathrm{~Hz}, 6 \mathrm{H}), 0.95(\operatorname{app} d, J=6.7 \mathrm{~Hz}, 6 \mathrm{H}), 0.79(\mathrm{~d}, J=6.7 \mathrm{~Hz}, 3 \mathrm{H}) ;{ }^{13} \mathrm{C}$ NMR $(125$ $\left.\mathrm{MHz}, \mathrm{CD}_{3} \mathrm{OD}\right) \square 166.2,155.4,147.5,146.7,132.8,132.7$ (2), 132.0 (2), 131.9, 130.1, 129.6, $126.2,120.5$ (br), 117.2, 113.5, 80.0, 79.3, 78.5 (br), 75.5, 62.1, 40.7, 40.1, 37.5, 36.3, 35.7, 35.3, 33.9, 33.8, 32.8, 21.9, 18.3, 16.8, 16.7, $15.3(2), 13.5,8.2$; high resolution mass spectrum $\left(\mathrm{ES}^{+}\right)$ $\mathrm{m} / \mathrm{z} 717.5421\left[(\mathrm{M}+\mathrm{Na})^{+}\right.$; calcd for $\left.\mathrm{C}_{41} \mathrm{H}_{62} \mathrm{~N}_{2} \mathrm{O}_{7} \mathrm{Na}: 717.4459\right]$.

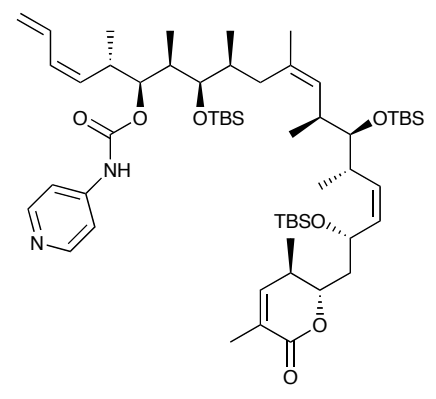

(+)-S13

Pyridine carbamate (+)-S13. Preparation for ca. $0.2 \mathrm{M}$ pyridyl isocyanate in benzene solution: A solution of isonicotinic acid $(2.0 \mathrm{~g}, 16.3 \mathrm{mmol})$ in DMF $(12 \mathrm{~mL})$ was treated with diphenylphosphoryl azide $(3.5 \mathrm{~mL}, 16.3 \mathrm{mmol})$ and triethyl amine $(2.27 \mathrm{~mL}, 16.3 \mathrm{mmol})$ at $35{ }^{\circ} \mathrm{C}$ for $2 \mathrm{hr}$. Quenched by pouring reaction mixture into $\mathrm{Et}_{2} \mathrm{O} / \mathrm{ice}$, extracted $2 \times 30 \mathrm{~mL}$ ether. Washed combined organics with saturated $\mathrm{NaHCO}_{3}(15 \mathrm{~mL})$ and water $(15 \mathrm{~mL})$. Dried over $\mathrm{MgSO}_{4}$, concentrated and redissolved in benzene. Heated solution to $80^{\circ} \mathrm{C}$ for $1.5 \mathrm{~h}$. and then cooled to room temperature to yield a $0.2 \mathrm{M}$ pyridyl isocyanate in benzene solution.

Carbamate formation: A solution of alcohol $(+)-19(15.0 \mathrm{mg}, 0.016 \mathrm{mmol})$ was dissolved in the above $0.2 \mathrm{M}$ pyridyl isocyanate solution $(1 \mathrm{~mL})$, warmed to $50{ }^{\circ} \mathrm{C}$, and stirred for $2 \mathrm{~h}$. The reaction mixture was applied directly to flash chromotography (10\% to $40 \%$ ethyl acetate/hexanes) to yield $8.5 \mathrm{mg}$ tri-TBS carbamate (+)-S13 (53\%) as a colorless oil in addition to recovered (+)-19 (36\%). 
$[\square]_{\mathrm{D}}^{23}+46.0\left(\mathrm{c} 0.5, \mathrm{CHCl}_{3}\right) ; \mathrm{IR}(\mathrm{NaCl}) 3325,2957,2929,2884,2857,1733,1652,1594,1521$, 1377, 1249, 1223, 1208, 1095, 1070, 835, $773 \mathrm{~cm}^{-1} ;{ }^{1} \mathrm{H}-\mathrm{NMR}\left(500 \mathrm{MHz}, \mathrm{CDCl}_{3}\right) \square 8.45$ (app s, 2H), 7.35 (app s, 2H), 7.00 (s, 1H), 6.61 (ddd, $J=16.8,10.8,10.8 \mathrm{~Hz}, 1 \mathrm{H}), 6.28$ (dd, $J=2.2,1.4$ $\mathrm{Hz}, 1 \mathrm{H}), 6.01(\mathrm{dd}, J=11.2,11.2 \mathrm{~Hz}, 1 \mathrm{H}), 5.35(\mathrm{dd}, J=10.4,10.4 \mathrm{~Hz}, 1 \mathrm{H}), 5.30-5.15(\mathrm{~m}, 4 \mathrm{H})$, $5.06(\mathrm{~d}, J=9.7 \mathrm{~Hz}, 1 \mathrm{H}), 4.86(\mathrm{dd}, J=5.9,5.9 \mathrm{~Hz}, 1 \mathrm{H}), 4.75(\mathrm{~m}, 1 \mathrm{H}), 4.20(\mathrm{ddd}, J=10.0,10.0$, $2.6 \mathrm{~Hz}, 1 \mathrm{H}$ ), $3.48(\mathrm{dd}, J=4.5,4.5 \mathrm{~Hz}, 1 \mathrm{H}), 3.28(\mathrm{dd}, J=5.2,5.2 \mathrm{~Hz}, 1 \mathrm{H}$ ), 3.06 (ddd, $J=10.0,6.7$, $6.7 \mathrm{~Hz}, 1 \mathrm{H}), 2.60(\mathrm{~m}, 1 \mathrm{H}), 2.44(\mathrm{dddd}, J=11.5,10.0,6.7,6.7 \mathrm{~Hz}, 1 \mathrm{H}), 2.36(\mathrm{~m}, 1 \mathrm{H}), 2.11(\mathrm{dd}, J=$ 12.3, $12.3 \mathrm{~Hz}, 1 \mathrm{H}), 1.97(\mathrm{~m}, 1 \mathrm{H}), 1.90(\mathrm{dd}, J=1.5,1.5 \mathrm{~Hz}, 3 \mathrm{H}), 1.90(\mathrm{~m}, 1 \mathrm{H}), 1.76-1.66(\mathrm{~m}, 3 \mathrm{H})$, $1.59(\mathrm{~s}, 3 \mathrm{H}), 1.05(\mathrm{~d}, J=7.1 \mathrm{~Hz}, 3 \mathrm{H}), 1.03(\mathrm{~d}, J=6.7 \mathrm{~Hz}, 3 \mathrm{H}), 0.98(\mathrm{~d}, J=6.7 \mathrm{~Hz}, 3 \mathrm{H}), 0.97(\mathrm{~d}, J$ $=6.7 \mathrm{~Hz}, 3 \mathrm{H}), 0.94(\mathrm{~s}, 9 \mathrm{H}), 0.91(\mathrm{~s}, 9 \mathrm{H}), 0.90(\mathrm{~d}, J=7.1 \mathrm{~Hz}, 3 \mathrm{H}), 0.85(\mathrm{~s}, 9 \mathrm{H}), 0.74(\mathrm{~d}, J=6.7 \mathrm{~Hz}$, 3H), $0.10(\mathrm{~s}, 3 \mathrm{H}), 0.083(\mathrm{~s}, 3 \mathrm{H}), 0.079(\mathrm{~s}, 3 \mathrm{H}), 0.05(\mathrm{~s}, 3 \mathrm{H}), 0.04(\mathrm{~s}, 3 \mathrm{H}), 0.03(\mathrm{~s}, 3 \mathrm{H}) ;{ }^{13} \mathrm{C}-\mathrm{NMR}$ $\left(125 \mathrm{MHz}, \mathrm{CDCl}_{3}\right) \square 165.3,152.8,150.1,145.7,145.6,132.94,132.89,132.4,132.0,131.9$, $131.3,130.1,127.2,118.4,112.4,80.5,80.0,79.8,16.8,64.5,41.6,37.59,37.57,35.8,35.7$ $35.4,34.4,33.9,26.2,26.1,25.8,23.0,18.40,18.39,18.01,17.45(2), 16.8,16.6,16.4,13.8$, $10.3,-3.15,-3.4,-3.7,-4.30,-4.34,-4.9$; high resolution mass spectrum $\left(E^{+}\right) \mathrm{m} / \mathrm{z} 995.6753$ $\left[(\mathrm{M}+\mathrm{H})^{+} ;\right.$calcd for $\left.\mathrm{C}_{56} \mathrm{H}_{99} \mathrm{~N}_{2} \mathrm{O}_{7} \mathrm{Si}_{3}: 995.6760\right]$

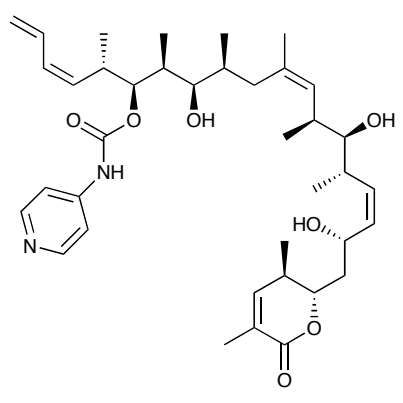

(+)-S22

Pyridine carbamate triol (+)-22: Tri-TBS carbamate (+)-S13 (8.5 mg, $7.6 \mu \mathrm{mol})$ was dissolved in $\mathrm{MeOH}(2.0 \mathrm{~mL})$. Aqueous $\mathrm{HCl}(4 \mathrm{~N}, 3.0 \mathrm{~mL})$ was added dropwise over $4 \mathrm{hr}$ at room temperature. The reaction mixture was stirred for $4 \mathrm{hr}$ further, diluted with water $(10 \mathrm{~mL})$ and EtOAc $(15 \mathrm{~mL})$. The resulting mixture was neutralized by solid $\mathrm{NaHCO}_{3}$, and separated. The aqueous layer was 
extracted with EtOAc $(3 \times 10 \mathrm{~mL})$. The combined organic layers were washed with saturated brine $(1 \times 10 \mathrm{~mL})$, dried over $\mathrm{MgSO}_{4}$, filtered, and concentrated. Flash chromotography ( $2 \%$ to $\left.5 \% \mathrm{MeOH} / \mathrm{CH}_{2} \mathrm{Cl}_{2}\right)$ provided 4-pyridine carbamate triol (+)-22 (4.9 $\left.\mathrm{mg}, 98 \%\right)$ as a colorless solid. $[\square]_{D}^{23}+39.8$ (c 0.5, MeOH); IR (film, NaCl) 3291, 2962, 2919, 2866, 1699, 1594, 1521, 1456, 1373, 1332, 1256, 1228, 1207, 1135, $1047 \mathrm{~cm}^{-1} ;{ }^{1} \mathrm{H}$ NMR $\left(500 \mathrm{MHz}, \mathrm{CD}_{3} \mathrm{OD}\right) \square 8.32(\mathrm{br} \mathrm{d}, J=$ $4.8 \mathrm{~Hz}, 2 \mathrm{H}$ ), 7.50 (br d, $J=4.8 \mathrm{~Hz}, 2 \mathrm{H}$ ), 6.70 (ddd, $J=16.7,10.4,10.4 \mathrm{~Hz}, 1 \mathrm{H}$ ), 6.34 (ddd, $J=$ 3.7, $1.5,1.5 \mathrm{~Hz}, 1 \mathrm{H}), 6.07$ (dd, $J=11.2,10.8 \mathrm{~Hz}, 1 \mathrm{H}$ ), 5.60 (dd, $J=10.8,10.8 \mathrm{~Hz}, 1 \mathrm{H}$ ), 5.46 (dd, $J=10.8,10.8 \mathrm{~Hz}, 1 \mathrm{H}), 5.37(\mathrm{dd}, J=11.2,8.9 \mathrm{~Hz}, 1 \mathrm{H}), 5.25(\mathrm{~d}, J=16.8 \mathrm{~Hz}, 1 \mathrm{H}), 5.16(\mathrm{~d}, J=10.1$ $\mathrm{Hz}, 1 \mathrm{H}), 5.01-4.96(\mathrm{~m}, 2 \mathrm{H}), 4.58(\mathrm{dddd}, J=9.7,8.6,2.2,1.1 \mathrm{~Hz}, 1 \mathrm{H}), 4.26(\mathrm{ddd}, J=10.4,10.4$, $2.2 \mathrm{~Hz}, 1 \mathrm{H}), 3.25(\mathrm{dd}, J=6.7,4.8 \mathrm{~Hz}, 1 \mathrm{H}), 3.24-3.17(\mathrm{~m}, 1 \mathrm{H}), 3.13(\mathrm{dd}, J=8.2,3.3 \mathrm{~Hz}, 1 \mathrm{H}), 2.74-$ $2.66(\mathrm{~m}, 1 \mathrm{H}), 2.42-2.30(\mathrm{~m}, 2 \mathrm{H}), 2.02-1.84(\mathrm{~m}, 3 \mathrm{H}), 1.81$ (dd, $J=2.2,1.5 \mathrm{~Hz}, 3 \mathrm{H}), 1.75$ (ddd, $J=$ 14.5, 10.8, 2.2 Hz, 1H), 1.70-1.62 (m, 2H), $1.61(\mathrm{~s}, 3 \mathrm{H}), 1.07$ (d, $J=7.1 \mathrm{~Hz}, 3 \mathrm{H}), 1.035$ (d, J = 7.1 $\mathrm{Hz}, 3 \mathrm{H}), 1.038(\mathrm{~d}, J=6.7 \mathrm{~Hz}, 3 \mathrm{H}), 0.98(\mathrm{~d}, J=6.7 \mathrm{~Hz}, 3 \mathrm{H}), 0.96(\mathrm{~d}, J=6.7 \mathrm{~Hz}, 3 \mathrm{H}), 0.81(\mathrm{~d}, J=$ $6.3 \mathrm{~Hz}, 3 \mathrm{H}) ;{ }^{13} \mathrm{C}$ NMR $\left(125 \mathrm{MHz}, \mathrm{CD}_{3} \mathrm{OD}\right) \square 166.2,153.9,149.1,147.5,146.5,132.8,132.5$, $132.4,131.9,131.8,130.2,129.85,126.4,117.4,112.5,80.0,79.5,79.2,75.4,62.2,40.7,37.3$, $36.2,35.7,35.4,33.9,33.8,32.8,22.0,18.2,16.60,16.57,15.3$ (2), 13.4, 8.2; high resolution mass spectrum (ES+) $\mathrm{m} / \mathrm{z} 653.4193\left[(\mathrm{M}+\mathrm{H})^{+}\right.$; calcd for $\left.\mathrm{C}_{38} \mathrm{H}_{56} \mathrm{~N}_{2} \mathrm{O}_{7}: 653.4166\right]$.

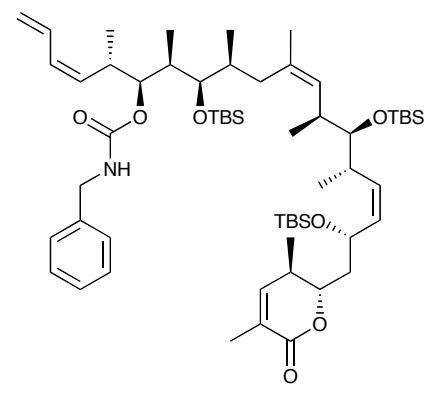

(+)-S14

Benzyl carbamate (+)-S14: A solution of alcohol (+)-19 (11 mg, $0.012 \mathrm{mmol})$ in $\mathrm{CH}_{2} \mathrm{Cl}_{2}(1.0 \mathrm{~mL})$ was treated with benzyl isocyanate $(45 \mu \mathrm{L}, 0.36 \mathrm{mmol})$ and $\mathrm{DBU}(28 \mu \mathrm{L}, 0.18 \mathrm{mmol})$ at room 
temperature for $1 \mathrm{hr}$, at which time benzyl isocyanate and DBU (45 $\mu \mathrm{L}$ and $28 \mu \mathrm{L}$, respectively) were added again. The reaction mixture was quenched with saturated $\mathrm{NH}_{4} \mathrm{Cl}(2 \mathrm{~mL})$, and diluted with $\mathrm{CH}_{2} \mathrm{Cl}_{2}(5 \mathrm{~mL})$. The resulting layers were separated, and the aqueous layer was extracted with $\mathrm{CH}_{2} \mathrm{Cl}_{2}(3 \times 5 \mathrm{~mL})$. The combined organic layers were washed with saturated brine $(1 \times 8$ $\mathrm{mL}$ ), dried over $\mathrm{Na}_{2} \mathrm{SO}_{4}$, filtered, and concentrated. Flash chromotography (5\% ethyl acetate/ hexanes) provided $8.4 \mathrm{mg}$ tri-TBS carbamate $(+)$-S14 (68\%) as a colorless oil. [प] $]_{\mathrm{D}}^{23}+50.2$ (c 0.5 , $\mathrm{CHCl}_{3}$ ); IR ( $\left.\mathrm{NaCl}\right) 3378,2957,2929,2884,2857,1723,1506,1472,1376,1252,1145,1043$, 835, $774 \mathrm{~cm}^{-1}$; ${ }^{1} \mathrm{H}-\mathrm{NMR}\left(500 \mathrm{MHz}, \mathrm{CDCl}_{3}\right.$ ) $\square 7.36$ (app s, $1 \mathrm{H}$ ), 7.32 (dd, $J=7.4,7.4 \mathrm{~Hz}, 2 \mathrm{H}$ ), 7.26 (dd, $J=7.4,7.4 \mathrm{~Hz}, 2 \mathrm{H}), 6.59$ (ddd, $J=16.8,10.8,10.8 \mathrm{~Hz}, 1 \mathrm{H}), 6.30(\mathrm{dd}, J=2.6,1.4 \mathrm{~Hz}, 1 \mathrm{H})$, $5.94(\mathrm{dd}, J=10.8,10.8 \mathrm{~Hz}, 1 \mathrm{H}), 5.36(\mathrm{dd}, J=10.4,10.4 \mathrm{~Hz}, 1 \mathrm{H}), 5.29-5.22(\mathrm{~m}, 2 \mathrm{H}), 5.18(\mathrm{~d}, J=$ $16.8 \mathrm{~Hz}, 1 \mathrm{H}$ ), $5.11(\mathrm{dd}, J=10.0,10.0 \mathrm{~Hz}, 1 \mathrm{H}), 5.02(\mathrm{~d}, J=9.7 \mathrm{~Hz}, 1 \mathrm{H}), 4.88(\mathrm{dd}, J=5.2,5.2 \mathrm{~Hz}$, 1H), 4.80-4.73 (m, 2H), 4.42-4.28 (m, 2H), 4.19 (ddd, $J=10.0,10.0,3.0 \mathrm{~Hz}, 1 \mathrm{H}), 3.45(\mathrm{br} \mathrm{s}, 1 \mathrm{H})$, $3.26(\mathrm{dd}, J=5.2,5.2 \mathrm{~Hz}, 1 \mathrm{H}), 3.09(\mathrm{~m}, 1 \mathrm{H}), 2.61$ (dddd, $J=13.8,6.7,6.7,6.7 \mathrm{~Hz}, 1 \mathrm{H}), 2.46(\mathrm{~m}$, 1H), 2.40-2.35 (m, 1H), $2.11(\mathrm{dd}, J=12.7,12.7 \mathrm{~Hz}, 1 \mathrm{H}), 1.94-1.80(\mathrm{~m}, 2 \mathrm{H}), 1.89(\mathrm{dd}, J=2.6,1.5$ $\mathrm{Hz}, 3 \mathrm{H}), 1.76-1.66(\mathrm{~m}, 3 \mathrm{H}), 1.58(\mathrm{~s}, 3 \mathrm{H}), 1.05(\mathrm{~d}, J=7.1 \mathrm{~Hz}, 3 \mathrm{H}), 1.01(\mathrm{~d}, J=6.3 \mathrm{~Hz}, 3 \mathrm{H}), 0.98$ (d, $J=6.7 \mathrm{~Hz}, 3 \mathrm{H}), 0.94(\mathrm{~s}, 9 \mathrm{H}), 0.94$ (d, obscured, 3H), $0.91(\mathrm{~s}, 9 \mathrm{H}), 0.88(\mathrm{~d}, J=6.3 \mathrm{~Hz}, 3 \mathrm{H})$, 0.86 (s, 9H), 0.73 (d, J = 7.1 Hz, 3H), 0.091 (s, 3H), 0.089 (br s, 3H), 0.079 (s, 3H), 0.06 (s, 3H), 0.05 (s, 3H), 0.04 (s, 3H); ${ }^{13} \mathrm{C}-\mathrm{NMR}\left(125 \mathrm{MHz}, \mathrm{CDCl}_{3}\right) \square 165.2,156.7,145.6,133.7,132.9,132.6$, 132.0, 131.6, 131.4, 129.7, 128.5, 128.2, 127.3 (2), 127.2, 117.8, 80.5, 79.7, 78.6, 76.8, 64.5, $44.9,41.7,37.8,37.5,36.0,35.4,35.2,34.5,33.9,26.18,26.16,25.8,22.9,18.4,18.3,18.0$, $17.5,17.0,16.8,16.7,16.6,13.6,10.0,-3.1,-3.5,-3.6,-4.3,-4.4,-4.9 ;$ high resolution mass spectrum $\left(\right.$ ES $\left.^{+}\right) \mathrm{m} / \mathrm{z} 1030.6786\left[(\mathrm{M}+\mathrm{Na})^{+}\right.$; calcd for $\left.\mathrm{C}_{58} \mathrm{H}_{101} \mathrm{NO}_{7} \mathrm{Si}_{3} \mathrm{Na:} 1030.6784\right]$ 


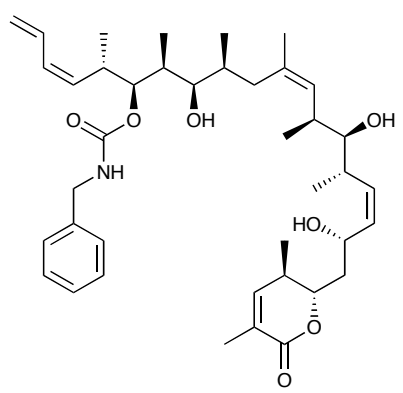

$(+)-23$

Benzyl carbamate triol (+)-23: Tri-TBS carbamate (+)-S14 (7.0 mg, $7.00 \mu \mathrm{mol})$ was dissolved in $\mathrm{MeOH}(2.5 \mathrm{~mL})$. Aqueous $\mathrm{HCl}(6 \mathrm{~N}, 2.0 \mathrm{~mL})$ was added dropwise over $4 \mathrm{hr}$ at room temperature. The reaction mixture was stirred for $4 \mathrm{hr}$ further, diluted with water $(6 \mathrm{~mL})$ and EtOAc $(10 \mathrm{~mL})$. The resulting mixture was neutralized by solid $\mathrm{NaHCO}_{3}$, and separated. The aqueous layer was extracted with EtOAc $(3 \times 8 \mathrm{~mL})$. The combined organic layers were washed with saturated brine (1 x $15 \mathrm{~mL}$ ), dried over $\mathrm{Na}_{2} \mathrm{SO}_{4}$, filtered, and concentrated. Flash chromotography (5\% $\left.\mathrm{MeOH} / \mathrm{CH}_{2} \mathrm{Cl}_{2}\right)$ provided benzyl carbamate triol $(+)-23(3.1 \mathrm{mg}, 67 \%)$ as a colorless oil. $[\square]_{\mathrm{D}}^{23}$ +54.0 (c 0.33, MeOH); IR (film, NaCl) 3420, 2965, 2925, 2872, 1703, 1516, 1455, 1376, 1250, 1140, 1038, 976, $902 \mathrm{~cm}^{-1} ;{ }^{1} \mathrm{H}$ NMR (500 MHz, CD $\left.{ }_{3} \mathrm{OD}\right)$ ] 7.33-7.17 (m, 5H), 6.68 (ddd, $J=16.7$, 10.4, 10.4 Hz, 1H), 6.36 (dd, $J=2.2,1.5 \mathrm{~Hz}, 1 \mathrm{H}), 6.06(\mathrm{dd}, J=11.2,10.8 \mathrm{~Hz}, 1 \mathrm{H}), 5.63(\mathrm{dd}, J=$ 10.4, 10.4 Hz, 1H), 5.45 (dd, $J=10.8,10.8 \mathrm{~Hz}, 1 \mathrm{H}), 5.40$ (dd, $J=11.2,8.9 \mathrm{~Hz}, 1 \mathrm{H}), 5.25(\mathrm{~d}, J=$ $16.8 \mathrm{~Hz}, 1 \mathrm{H}), 5.16(\mathrm{~d}, J=10.1 \mathrm{~Hz}, 1 \mathrm{H}), 4.96(\mathrm{~d}, J=10.1 \mathrm{~Hz}, 1 \mathrm{H}), 4.84(\mathrm{dd}, J=7.8,3.7 \mathrm{~Hz}, 1 \mathrm{H})$, $4.76(\mathrm{~m}, 1 \mathrm{H}), 4.57(\mathrm{dd}, J=9.3,9.3 \mathrm{~Hz}, 1 \mathrm{H}), 4.32(\mathrm{~d}, J=15.3 \mathrm{~Hz}, 1 \mathrm{H}), 4.24(\mathrm{~d}, J=15.3 \mathrm{~Hz}, 1 \mathrm{H})$, 4.33-4.22 (m, 1H), 3.22 (dd, $J=7.1,3.7 \mathrm{~Hz}, 1 \mathrm{H}), 3.19-3.12(\mathrm{~m}, 1 \mathrm{H}), 3.11(\mathrm{dd}, J=8.6,3.0 \mathrm{~Hz}$, 1H), 2.76-2.69 (m, 1H), 2.46-2.37 (m, 1H), 2.37-2.28 (m, 1H), $1.83(\mathrm{dd}, J=1.9,1.9 \mathrm{~Hz}, 3 \mathrm{H}), 1.89-$ $1.65(\mathrm{~m}, 5 \mathrm{H}), 1.63(\mathrm{~s}, 3 \mathrm{H}), 1.07(\mathrm{~d}, J=7.1 \mathrm{~Hz}, 3 \mathrm{H}), 1.05(\mathrm{~d}, J=7.1 \mathrm{~Hz}, 3 \mathrm{H}), 1.00(\mathrm{~d}, J=6.7 \mathrm{~Hz}$, $3 \mathrm{H}), 0.94(\mathrm{~d}, J=6.7 \mathrm{~Hz}, 3 \mathrm{H}), 0.86(\mathrm{~d}, J=6.7 \mathrm{~Hz}, 3 \mathrm{H}), 0.79(\mathrm{~d}, J=6.7 \mathrm{~Hz}, 3 \mathrm{H}) ;{ }^{13} \mathrm{C}$ NMR $(125$ $\left.\mathrm{MHz}, \mathrm{CD}_{3} \mathrm{OD}\right) \square 166.1,158.1,146.6,139.5,133.23,132.83,132.6,131.9,131.8,130.0,129.6$ $128.0,126.7,126.6,126.4,117.3,80.0,79.3,79.0,75.2,62.0,43.9,40.8,37.6,36.3,35.3,35.1$, 
33.9, 33.7, 33.1, 21.9, 18.3, 16.8, 16.6, 15.4 (2), 14.2, 8.0; high resolution mass spectrum (ES+) $m / z 688.4167\left[(\mathrm{M}+\mathrm{Na})^{+} ;\right.$calcd for $\left.\mathrm{C}_{40} \mathrm{H}_{59} \mathrm{NO}_{7} \mathrm{Na}: 688.4189\right]$.

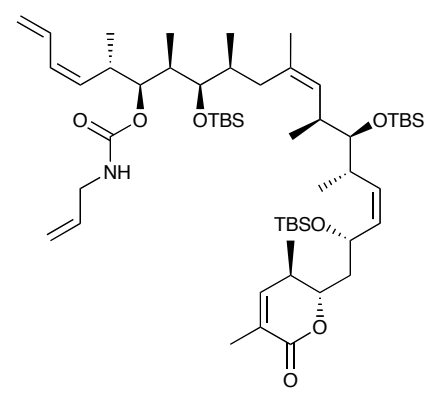

$(+)-S 15$

Allyl carbamate (+)-S15: A solution of alcohol (+)-19 (11 mg, $0.012 \mathrm{mmol})$ in $\mathrm{CH}_{2} \mathrm{Cl}_{2}(1 \mathrm{~mL})$ was treated with allyl isocyanate $(33 \mu \mathrm{L}, 0.36 \mathrm{mmol})$ and $\mathrm{DBU}(28 \mu \mathrm{L}, 0.18 \mathrm{mmol})$ at room temperature for $2 \mathrm{hr}$. The reaction mixture was quenched with saturated $\mathrm{NH}_{4} \mathrm{Cl}(2 \mathrm{~mL})$, and diluted with $\mathrm{CH}_{2} \mathrm{Cl}_{2}(5 \mathrm{~mL})$. The resulting layers were separated, and the aqueous layer was extracted with $\mathrm{CH}_{2} \mathrm{Cl}_{2}(3 \times 5 \mathrm{~mL})$. The combined organic layers were washed with saturated brine $(1 \times 8 \mathrm{~mL})$, dried over $\mathrm{Na}_{2} \mathrm{SO}_{4}$, filtered, and concentrated. Flash chromotography ( $5 \%$ ethyl acetate/ hexanes) provided $9.0 \mathrm{mg}$ tri-TBS carbamate $(+)-\mathbf{S} 15(82 \%)$ as a colorless oil. $[\square]_{\mathrm{D}}^{23}$ +53.6 (c 0.5, $\left.\mathrm{CHCl}_{3}\right)$; IR (NaCl) 3364, 2957, 2929, 2889, 2856, 1725, 1506, 1472, 1462, 1387, 1376, 1360, 1252, 1137, 1095, 836, $774 \mathrm{~cm}^{-1} ;{ }^{1} \mathrm{H}-\mathrm{NMR}\left(500 \mathrm{MHz}, \mathrm{CDCl}_{3}\right) \square 6.60(\mathrm{ddd}, J=16.8$, $10.8,10.8 \mathrm{~Hz}, 1 \mathrm{H}), 6.31(\mathrm{~s}, 1 \mathrm{H}), 6.01(\mathrm{dd}, J=10.8,10.8 \mathrm{~Hz}, 1 \mathrm{H}), 5.86-5.78(\mathrm{~m}, 1 \mathrm{H}), 5.38(\mathrm{dd}, J=$ 10.0, 10.0 Hz, 1H), 5.30-5.22 (m, 2H), 5.22-5.18 (m, 2H), 5.17-5.08 (m, 2H), $5.02(\mathrm{~d}, J=10.0 \mathrm{~Hz}$, 1H), $4.76(\mathrm{~m}, 2 \mathrm{H}), 4.64(\mathrm{br} \mathrm{s}, 1 \mathrm{H}), 4.19(\mathrm{ddd}, J=10.0,10.0,3.0 \mathrm{~Hz}, 1 \mathrm{H}), 3.77(\mathrm{~m}, 2 \mathrm{H}), 3.44(\mathrm{dd}, J$ $=4.1,4.1 \mathrm{~Hz}, 1 \mathrm{H}), 3.26(\mathrm{dd}, J=5.2,5.2 \mathrm{~Hz}, 1 \mathrm{H}), 3.03-2.96(\mathrm{~m}, 1 \mathrm{H}), 2.64-2.57(\mathrm{~m}, 1 \mathrm{H}), 2.49-2.42$ (m, 1H), 2.42-2.34 (m, 1H), $2.10(\mathrm{dd}, J=12.3,12.3 \mathrm{~Hz}, 1 \mathrm{H}), 1.90(\mathrm{br} \mathrm{s}, 3 \mathrm{H}), 1.90-1.80(\mathrm{~m}, 2 \mathrm{H})$, 1.75-1.62 (m, 3H), 1.58 (s, 3H), $1.06(\mathrm{~d}, J=7.1 \mathrm{~Hz}, 3 \mathrm{H}), 1.00$ (d, J= $7.1 \mathrm{~Hz}, 3 \mathrm{H}), 0.97$ (d, J=6.7 $\mathrm{Hz}, 3 \mathrm{H}), 0.93$ (s, 9H), 0.92 (d, obscured, 3H), 0.91 (s, 9H), 0.88 (d, J = 6.3 Hz, 3H), $0.86(\mathrm{~s}, 9 \mathrm{H})$, $0.72(\mathrm{~d}, J=7.1 \mathrm{~Hz}, 3 \mathrm{H}), 0.11(\mathrm{~s}, 3 \mathrm{H}), 0.09(\mathrm{~s}, 3 \mathrm{H}), 0.08(\mathrm{~s}, 3 \mathrm{H}), 0.06(\mathrm{~s}, 3 \mathrm{H}), 0.05(\mathrm{~s}, 3 \mathrm{H}), 0.04$ (s, $3 \mathrm{H}) ;{ }^{13} \mathrm{C}-\mathrm{NMR}\left(125 \mathrm{MHz}, \mathrm{CDCl}_{3}\right) \square 165.2,156.5,145.2,134.7,137.6,132.9,132.6,132.0,131.6$, 
$131.4,129.6,127.2,117.85,115.6,80.5,79.7,78.5,77.0,64.5,43.3,41.7,37.9,37.4,35.9,35.4$ (2), 34.5, 33.9, 26.17, 26.16, 25.8, 22.9, 18.44, 18.36, 18.0, 17.5, 17.1, 16.8, 16.7, 16.6, 13.2, $10.1,-3.1,-3.51,-3.56,-4.35,-4.37,-4.90$; high resolution mass spectrum (ES ${ }^{+}$m/z 980.6629 $\left[(\mathrm{M}+\mathrm{Na})^{+}\right.$; calcd for $\left.\mathrm{C}_{54} \mathrm{H}_{99} \mathrm{NO}_{7} \mathrm{Si}_{3} \mathrm{Na}: 980.6627\right]$

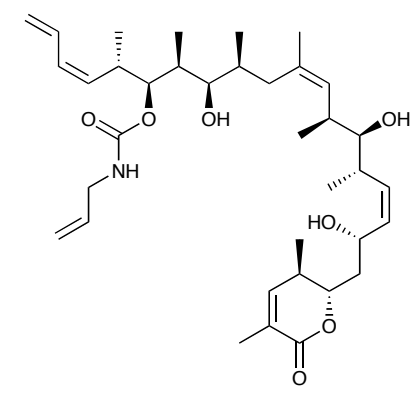

(+)-24

Allyl carbamate triol (+)-24: Tri-TBS carbamate (+)-S15 (5.5 mg, $5.6 \mu \mathrm{mol})$ was dissolved in $\mathrm{MeOH}(0.7 \mathrm{~mL})$. Aqueous $\mathrm{HCl}(6 \mathrm{~N}, 2.0 \mathrm{~mL})$ was added dropwise over $4 \mathrm{hr}$ at room temperature. The reaction mixture was stirred for $4 \mathrm{hr}$ further, diluted with water $(10 \mathrm{~mL})$ and EtOAc $(15 \mathrm{~mL})$. The resulting mixture was neutralized by solid $\mathrm{NaHCO}_{3}$, and separated. The aqueous layer was extracted with EtOAc $(3 \times 10 \mathrm{~mL})$. The combined organic layers were washed with saturated brine $(1 \times 15 \mathrm{~mL})$, dried over $\mathrm{Na}_{2} \mathrm{SO}_{4}$, filtered, and concentrated. Flash chromotography $(2 \%$ to $\left.5 \% \mathrm{MeOH} / \mathrm{CH}_{2} \mathrm{Cl}_{2}\right)$ provided allyl carbamate triol (+)-24 (3.2 mg, 92\%) as a colorless solid. $[\square]_{\mathrm{D}}^{23}$ +45.3 (c 0.3, $\left.\mathrm{CHCl}_{3}\right)$; IR (film, NaCl) 3433, 2965, 2931, 2872, 1701, 1507, 1457, 1367, 1250, 1141, 1036, $983 \mathrm{~cm}^{-1} ;{ }^{1} \mathrm{H}$ NMR (500 MHz, $\left.\mathrm{CD}_{3} \mathrm{OD}\right) \square 6.68$ (ddd, $J=16.8,10.4,10.4 \mathrm{~Hz}, 1 \mathrm{H}$ ), 6.49 (ddd, $J=3.7,1.5,1.5 \mathrm{~Hz}, 1 \mathrm{H}$ ), 6.07 (dd, $J=11.2,10.8 \mathrm{~Hz}, 1 \mathrm{H}$ ), 5.84 (ddddd, $J=15.6,10.1$, 4.8, 4.8, 2.2 Hz, 1H), 5.62 (dd, $J=10.8,10.8 \mathrm{~Hz}, 1 \mathrm{H}$ ), 5.45 (dd, $J=10.8,10.8 \mathrm{~Hz}, 1 \mathrm{H}$ ), 5.39 (dd, $J=10.8,8.9 \mathrm{~Hz}, 1 \mathrm{H}), 5.27(\mathrm{dd}, J=16.7,1.9 \mathrm{~Hz}, 1 \mathrm{H}), 5.20-5.14(\mathrm{~m}, 2 \mathrm{H}), 5.07(\mathrm{~d}, J=10.1 \mathrm{~Hz}$, 1H), $4.97(\mathrm{~d}, J=10.1 \mathrm{~Hz}, 1 \mathrm{H}), 4.88-4.82(\mathrm{~m}, 1 \mathrm{H}), 4.58$ (dddd, $J=9.7,8.6,2.2,1.1 \mathrm{~Hz}, 1 \mathrm{H}), 4.29$ (ddd, $J=10.4,10.4,2.2 \mathrm{~Hz}, 1 \mathrm{H}$ ), 3.70 (dd, $J=3.3,1.5 \mathrm{~Hz}, 2 \mathrm{H}$ ), 3.22 (dd, $J=7.8,3.7 \mathrm{~Hz}, 1 \mathrm{H}$ ), 3.18-3.12 (m, 1H), 3.12 (dd, $J=8.6,3.0 \mathrm{~Hz}, 1 \mathrm{H}), 2.75-2.68(\mathrm{~m}, 1 \mathrm{H}), 2.50-2.41(\mathrm{~m}, 1 \mathrm{H}), 2.39-2.29$ (m, 1H), 1.86 (dd, $J=2.2,1.5 \mathrm{~Hz}, 3 \mathrm{H}), 1.84-1.75(\mathrm{~m}, 4 \mathrm{H}), 1.68$ (ddd, $J=14.1,10.8,2.2 \mathrm{~Hz}, 2 \mathrm{H})$, 
$1.64(\mathrm{~s}, 3 \mathrm{H}), 1.12$ (d, J=7.1 Hz, 3H), 1.07 (d, J = 7.1 Hz, 3H), 0.99 (d, J = 6.7 Hz, 3H), 0.94 (d, J $=6.7 \mathrm{~Hz}, 3 \mathrm{H}), 0.86(\mathrm{~d}, J=6.7 \mathrm{~Hz}, 3 \mathrm{H}), 0.79(\mathrm{~d}, J=6.3 \mathrm{~Hz}, 3 \mathrm{H}) ;{ }^{13} \mathrm{C}$ NMR $\left(125 \mathrm{MHz}, \mathrm{CD}_{3} \mathrm{OD}\right) \square$ $166.2,157.9,146.6,134.9,133.1,132.7,132.6,131.9,131.8,130.1,129.6,126.4,117.3,114.0$ $80.0,79.3,79.0,75.2,62.1,42.6,40.8,37.6,36.2,35.3,35.2,33.9,33.6,33.1,21.9,18.3,16.8$ 16.6, 15.4, 15.3, 14.2, 7.9; high resolution mass spectrum $\left(\mathrm{ES}^{+}\right) \mathrm{m} / \mathrm{z} 638.4021\left[(\mathrm{M}+\mathrm{Na})^{+}\right.$; calcd for $\mathrm{C}_{36} \mathrm{H}_{57} \mathrm{NO}_{7} \mathrm{Na:}$ 638.4033].

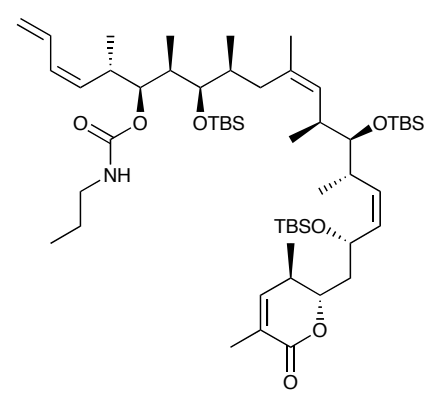

(+)-S16

n-Propyl carbamate (+)-S16: A solution of alcohol (+)-19 $(8.2 \mathrm{mg}, 0.011 \mathrm{mmol})$ in $\mathrm{CH}_{2} \mathrm{Cl}_{2}(1.0$ $\mathrm{mL}$ ) was treated with $n$-propyl isocyanate $(35 \mu \mathrm{L}, 0.33 \mathrm{mmol})$ and DBU $(25 \mu \mathrm{L}, 0.016 \mathrm{mmol})$ at room temperature for $22 \mathrm{hr}$. The reaction mixture was quenched with saturated $\mathrm{NH}_{4} \mathrm{Cl}(2 \mathrm{~mL})$, and diluted with $\mathrm{CH}_{2} \mathrm{Cl}_{2}(5 \mathrm{~mL})$. The resulting layers were separated, and the aqueous layer was extracted with $\mathrm{CH}_{2} \mathrm{Cl}_{2}(3 \times 5 \mathrm{~mL})$. The combined organic layers were washed with saturated brine $(1 \times 8 \mathrm{~mL})$, dried over $\mathrm{Na}_{2} \mathrm{SO}_{4}$, filtered, and concentrated. Preparative TLC (10\% ethyl acetate/ hexanes) provided $7.8 \mathrm{mg}$ tri-TBS carbamate (+)-S16 (85\%) as a colorless oil. $[\square]_{\mathrm{D}}^{23}$ +45.5 (c 0.4, $\left.\mathrm{CHCl}_{3}\right) ; \mathrm{IR}(\mathrm{NaCl}) 3356,2959,2925,2884,2855,1724,1512,1472,1372,1252$, 1041, 1001, 836, 774, $670 \mathrm{~cm}^{-1}$; ${ }^{1} \mathrm{H}-\mathrm{NMR}\left(500 \mathrm{MHz}, \mathrm{CDCl}_{3}\right) \square 6.60$ (ddd, $J=16.8,10.8,10.8 \mathrm{~Hz}$, 1H), $6.31(\mathrm{~s}, 1 \mathrm{H}), 6.01$ (dd, $J=10.4,10.4 \mathrm{~Hz}, 1 \mathrm{H}), 5.37$ (dd, $J=10.4,10.4 \mathrm{~Hz}, 1 \mathrm{H}), 5.30-5.20$ (m, 3H), $5.13(\mathrm{~d}, J=10.0 \mathrm{~Hz}, 1 \mathrm{H}), 5.02(\mathrm{~d}, J=9.7 \mathrm{~Hz}, 1 \mathrm{H}), 4.80-4.70(\mathrm{~m}, 2 \mathrm{H}), 4.59-4.52(\mathrm{~m}, 1 \mathrm{H})$, 4.20 (ddd, $J=10.0,10.0,2.6 \mathrm{~Hz}, 1 \mathrm{H}$ ), 3.44 (dd, $J=4.1,4.1 \mathrm{~Hz}, 1 \mathrm{H}$ ), 3.26 (dd, $J=5.2,5.2 \mathrm{~Hz}$, 1H), 3.16-3.06 (m, 2H), 3.01-2.95 (m, 1H), $2.61(\mathrm{ddd}, J=6.7,6.7,6.7 \mathrm{~Hz}, 1 \mathrm{H}), 2.48-2.42(\mathrm{~m}, 1 \mathrm{H})$, 
2.41-2.35 (m, 1H), $2.10(\mathrm{dd}, J=11.5,11.5 \mathrm{~Hz}, 1 \mathrm{H}), 1.90(\mathrm{~s}, 3 \mathrm{H}), 1.90-1.80(\mathrm{~m}, 1 \mathrm{H}), 1.75-1.62(\mathrm{~m}$, 4H), 1.54 (s, 3H), 1.49 (dddd, $J=14.5,7.4,7.4,7.4 \mathrm{~Hz}, 2 \mathrm{H}), 1.07$ (d, $J=7.1 \mathrm{~Hz}, 3 \mathrm{H}), 0.99$ (d, $J=$ $7.1 \mathrm{~Hz}, 3 \mathrm{H}$ ), 0.97 (d, $J=6.7 \mathrm{~Hz}, 3 \mathrm{H}), 0.93$ (s, 9H), 0.92 (obscured, 3H), 0.91 (s, 9H), 0.90 (d, $J=$ $6.3 \mathrm{~Hz}, 3 \mathrm{H}), 0.88(\mathrm{~d}, J=6.3 \mathrm{~Hz}, 3 \mathrm{H}), 0.86(\mathrm{~s}, 9 \mathrm{H}), 0.72(\mathrm{~d}, J=6.7 \mathrm{~Hz}, 3 \mathrm{H}), 0.10(\mathrm{~s}, 3 \mathrm{H}), 0.080$ (s, $3 \mathrm{H}), 0.078(\mathrm{~s}, 3 \mathrm{H}), 0.058(\mathrm{~s}, 3 \mathrm{H}), 0.046(\mathrm{~s}, 3 \mathrm{H}), 0.032(\mathrm{~s}, 3 \mathrm{H}) ;{ }^{13} \mathrm{C}-\mathrm{NMR}\left(125 \mathrm{MHz}, \mathrm{CDCl}_{3}\right)$ $165.2,156.7,145.6,133.8,132.9,132.6,132.1,131.6,131.5,129.6,127.2,117.7,80.4,79.7$, $78.2,77.0,64.5,42.7,41.7,37.8,37.4,35.9,35.4,34.5$ (2), 33.9, 26.2, 26.1, 25.8, 23.2, 22.8, $18.44,18.36,18.0,17.5,17.1,16.8,16.7,16.6,13.8,11.0,10.0,-3.1,-3.5,-3.6,-4.35,-4.37,-4.9$; high resolution mass spectrum $\left(\mathrm{ES}^{+}\right) \mathrm{m} / \mathrm{z} 982.6765\left[(\mathrm{M}+\mathrm{Na})^{+}\right.$; calcd for $\mathrm{C}_{54} \mathrm{H}_{101} \mathrm{NO}_{7} \mathrm{Si}_{3} \mathrm{Na}$ : 982.6783]

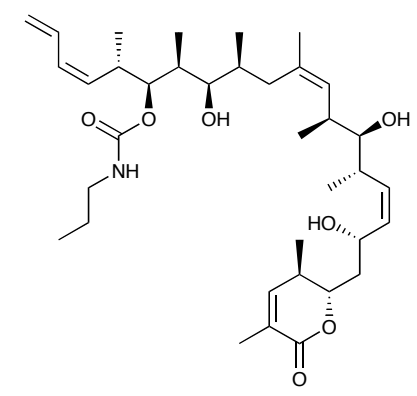

$(+)-25$

n-Propyl carbamate triol (+)-25: Tri-TBS carbamate (+)-S16 (19 mg, $19.5 \mu \mathrm{mol})$ was dissolved in $\mathrm{MeOH}(4 \mathrm{~mL})$. Aqueous $\mathrm{HCl}(6 \mathrm{~N}, 5 \mathrm{~mL})$ was added dropwise over $2 \mathrm{hr}$ at room temperature. The reaction mixture was stirred for $4 \mathrm{hr}$ further, diluted with water $(15 \mathrm{~mL})$ and EtOAc $(25 \mathrm{~mL})$. The resulting mixture was neutralized by solid $\mathrm{NaHCO}_{3}$, and separated. The aqueous layer was extracted with EtOAc $(3 \times 15 \mathrm{~mL})$. The combined organic layers were washed with saturated brine $(1 \times 15 \mathrm{~mL})$, dried over $\mathrm{MgSO}_{4}$, filtered, and concentrated. Preparative TLC (10\% $\mathrm{MeOH} / \mathrm{CH}_{2} \mathrm{Cl}_{2}$ ) provided $n$-propyl carbamate triol (+)-25 (10.4 mg, 86\%) as a colorless oil. $[\square]_{\mathrm{D}}^{23}$ +37.7 (c 1.0, MeOH); IR (film, NaCl) 3427, 2965, 2931, 2874, 1700, 1516, 1455, 1378, 1264, 1141, 1037, 975, $907 \mathrm{~cm}^{-1} ;{ }^{1} \mathrm{H}$ NMR (500 MHz, CD $\left.{ }_{3} \mathrm{OD}\right) \square 6.67$ (ddd, $J=16.8,10.4,10.4 \mathrm{~Hz}$, 
1H), 6.49 (ddd, $J=3.7,1.5,1.5 \mathrm{~Hz}, 1 \mathrm{H}), 6.06(\mathrm{dd}, J=11.2,10.8 \mathrm{~Hz}, 1 \mathrm{H}), 5.62(\mathrm{dd}, J=10.8,10.8$ $\mathrm{Hz}, 1 \mathrm{H}), 5.45(\mathrm{dd}, J=10.8,10.8 \mathrm{~Hz}, 1 \mathrm{H}), 5.39(\mathrm{dd}, J=11.2,8.9 \mathrm{~Hz}, 1 \mathrm{H}), 5.25(\mathrm{~d}, J=16.8 \mathrm{~Hz}$, 1H), $5.16(\mathrm{~d}, J=10.1 \mathrm{~Hz}, 1 \mathrm{H}), 4.97(\mathrm{~d}, J=10.1 \mathrm{~Hz}, 1 \mathrm{H}), 4.88-4.82(\mathrm{~m}, 1 \mathrm{H}), 4.58$ (dddd, $J=9.7$, 8.6, 2.2, 1.1 Hz, 1H), 4.29 (ddd, $J=10.4,10.4,2.2 \mathrm{~Hz}, 1 \mathrm{H}$ ), 3.21 (dd, $J=7.8,3.7 \mathrm{~Hz}, 1 \mathrm{H}$ ), 3.18$3.10(\mathrm{~m}, 1 \mathrm{H}), 3.12(\mathrm{dd}, J=8.6,3.0 \mathrm{~Hz}, 1 \mathrm{H}), 3.05(\mathrm{dd}, J=7.1,7.1 \mathrm{~Hz}, 2 \mathrm{H}), 2.76-2.68(\mathrm{~m}, 1 \mathrm{H})$, 2.49-2.41 (m, 1H), 2.39-2.30 (m, 1H), $1.86(\mathrm{dd}, J=2.2,1.5 \mathrm{~Hz}, 3 \mathrm{H}), 1.87-1.75(\mathrm{~m}, 4 \mathrm{H}), 1.67$ (ddd, $J=14.5,10.4,2.2 \mathrm{~Hz}, 2 \mathrm{H}), 1.63(\mathrm{br} \mathrm{s}, 3 \mathrm{H}), 1.50(\mathrm{~m}, 2 \mathrm{H}), 1.13(\mathrm{~d}, J=7.1 \mathrm{~Hz}, 3 \mathrm{H}), 1.08$ (d, $J=7.1$ $\mathrm{Hz}, 3 \mathrm{H}), 0.99$ (d, J = 7.1 Hz, 3H), 0.95 (d, J = 6.7 Hz, 3H), 0.91 (dd, J = 7.4, 7.4 Hz, 3H), 0.86 (d, $J=6.7 \mathrm{~Hz}, 3 \mathrm{H}), 0.79(\mathrm{~d}, J=6.3 \mathrm{~Hz}, 3 \mathrm{H}) ;{ }^{13} \mathrm{C} \mathrm{NMR}\left(125 \mathrm{MHz}, \mathrm{CD}_{3} \mathrm{OD}\right) \square 166.1,158.1,146.6$, $133.1,132.7,132.6,131.9,131.8,130.1,129.5,126.4,117.3,80.0,79.3,78.6,75.2,62.1,42.1$, $40.8,37.6,36.2,35.3,35.2,33.9,33.6,33.1,22.7,21.9,18.3,16.7,16.6,15.42,15.37,14.2$, 10.1, 7.9; high resolution mass spectrum $\left(\mathrm{ES}^{+}\right) \mathrm{m} / \mathrm{z} 640.4189\left[(\mathrm{M}+\mathrm{Na})^{+}\right.$; calcd for $\mathrm{C}_{36} \mathrm{H}_{59} \mathrm{NO}_{7} \mathrm{Na}$ : 640.4189].

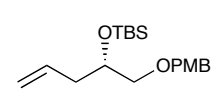

$(-)-S 17$

Alkene (-)-S17. A solution of alcohol (-)-26 (0.768 g, $4 \mathrm{mmol})$ and imidazole $(0.544 \mathrm{~g}, 8.0 \mathrm{mmol})$ in $\mathrm{CH}_{2} \mathrm{Cl}_{2}(40 \mathrm{~mL})$ was cooled to $0{ }^{\circ} \mathrm{C}$ and treated with tert-butyldimethylsilyl chloride $(900 \mathrm{mg}, 6$ $\mathrm{mmol})$. The resultant solution was stirred $12 \mathrm{~h}$ at ambient temperature, diluted with ether $(75 \mathrm{~mL})$, washed with $\mathrm{H}_{2} \mathrm{O}(2 \times 100 \mathrm{~mL})$ and saturated brine $(100 \mathrm{~mL})$, dried over $\mathrm{MgSO}_{4}$, and concentrated. Flash chromatography (5\% ethyl acetate/hexanes) afforded alkene (-)-S17 (1.18 g, 95\%) as a colorless oil. [ []$_{\mathrm{D}}^{23}-25^{\circ}\left(\mathrm{c} 0.48, \mathrm{CHCl}_{3}\right)$; IR (neat) 2954, 2928, 2897, 2856, 1613, 1514, 1472, 1249, 1105, 1038, $835 \mathrm{~cm}^{-1} ;{ }^{1} \mathrm{H}$ NMR $\left(500 \mathrm{MHz}, \mathrm{CDCl}_{3}\right) \square 7.26(\mathrm{~d}, J=8.6 \mathrm{~Hz}, 2 \mathrm{H}), 6.88(\mathrm{~d}$, $J=8.6 \mathrm{~Hz}, 2 \mathrm{H}), 5.83(\mathrm{dddd}, J=17.0,10.2,7.2,7.2 \mathrm{~Hz}, 1 \mathrm{H}), 5.06(\mathrm{~d}, J=17.0 \mathrm{~Hz}, 1 \mathrm{H}), 5.03(\mathrm{~d}, J$ $=7.8 \mathrm{~Hz}, 1 \mathrm{H}), 4.47(\mathrm{~d}, J=12.1 \mathrm{~Hz}, 1 \mathrm{H}), 4.44(\mathrm{~d}, J=12.1 \mathrm{~Hz}, 1 \mathrm{H}), 3.88$ (ddd, $J=11.2,5.8,5.6$ $\mathrm{Hz}, 1 \mathrm{H}), 3.81(\mathrm{~s}, 3 \mathrm{H}), 3.38(\mathrm{dd}, J=9.6,5.6 \mathrm{~Hz}, 1 \mathrm{H}), 3.36(\mathrm{dd}, J=9.6,5.6 \mathrm{~Hz}, 1 \mathrm{H}), 2.24(\mathrm{ddd}, J=$ 13.4, 6.3, 6.3 Hz, 1H), 2.21 (ddd, $J=13.4,6.3,6.3 \mathrm{~Hz}, 1 \mathrm{H}$ ), $0.90(\mathrm{~s}, 9 \mathrm{H}), 0.06$ (s, 3H), 0.06 (s, 
$3 \mathrm{H}) ;{ }^{13} \mathrm{C}$ NMR $\left(125 \mathrm{MHz}, \mathrm{CDCl}_{3}\right) \square 159.1,134.9,130.6,129.1,116.9,113.7,73.9,72.9,71.2$, 55.2, 39.3, 25.8, 18.1, -4.5, -4.8; $\left(\mathrm{ES}^{+}\right) \mathrm{m} / \mathrm{z} 335.202935\left[(\mathrm{M}+\mathrm{Na})^{+}\right.$; calcd for $\mathrm{C}_{19} \mathrm{H}_{32} \mathrm{NaO}_{3} \mathrm{Si}$ : $335.204249]$

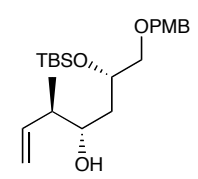

$(-)-27$

Alcohol (-)-27. Alkene (-)-S17 (1.7g, $5.5 \mathrm{mmol})$ was dissolved in $\mathrm{CH}_{2} \mathrm{Cl}_{2}(55 \mathrm{~mL})$ and bubbled $\mathrm{O}_{3}$ at $-78^{\circ} \mathrm{C}$ for 20 minutes, then the solution was degassed with argon and $\mathrm{PPh}_{3}$ (excess) was added. After 1 hour the mixture was evaporated and filtered quickly to provide intermediate aldehyde (-)-S18. In another flask, $n$-BuLi (2.4 M in hexanes, $3.3 \mathrm{~mL})$ was added dropwise to a cloudy solution of potassium tert-butoxide $(925.8 \mathrm{mg}, 8.25 \mathrm{mmol})$ and trans-2-butene (excess, 1 $\mathrm{mL})$ in THF $(12 \mathrm{~mL})$ at $-78{ }^{\circ} \mathrm{C}$. The resulting yellow mixture was stirred at $-45^{\circ} \mathrm{C}$ for $20 \mathrm{~min}$. The reaction mixture was recooled to $-78{ }^{\circ} \mathrm{C}$ and a solution of (+)-- -methoxydiisopinocampheylborane (2.96 g, $9.35 \mathrm{mmol})$ in THF $(5 \mathrm{~mL})$ was added. The resulting colorless reaction mixture was stirred at $-78{ }^{\circ} \mathrm{C}$ for $35 \mathrm{~min} . \mathrm{BF}_{3} \cdot \mathrm{Et}_{2} \mathrm{O}(1.4 \mathrm{~mL}, 11 \mathrm{mmol})$ was added rapidly followed immediately by a solution of the above aldehyde (-)-S18 $(1.7 \mathrm{~g}, 5.5 \mathrm{mmol})$ in $2.5 \mathrm{~mL}$ of THF. The resulting cloudy reaction mixture was stirred at $-78^{\circ} \mathrm{C}$ for $4 \mathrm{~h}$. The reaction was then quenched by addition of $3 \mathrm{~N}$ aqueous $\mathrm{NaOH}(5 \mathrm{~mL})$ followed by $30 \%$ aqueous $\mathrm{H}_{2} \mathrm{O}_{2}(5 \mathrm{~mL})$. The reaction mixture was warmed to $25{ }^{\circ} \mathrm{C}$ and stirred overnight. The mixture was diluted with ethyl acetate and saturated aqueous $\mathrm{NaCl}$. The layers were separated and the aqueous layer was extracted with ethyl acetate. The combined organic extracts were dried $\left(\mathrm{MgSO}_{4}\right)$ and then concentrated in vacuo. The homoallylic alcohols were purified by very careful flash column chromatography to obtain alcohol (-)-27 (1.21 g, 75\% yield). [ []$_{D}^{23}-12.2$ (c 0.36); IR (neat) 3474, 2957, 2929, 2856, 1514, 1472, 1250, 1172, 1089, 1036, $836 \mathrm{~cm}^{-1} ;{ }^{1} \mathrm{H}$ NMR $\left(500 \mathrm{MHz}, \mathrm{CDCl}_{3}\right) \square 7.24$ (d, J = 8.6 Hz, 2H), 6.86 (d, $J=8.6 \mathrm{~Hz}, 2 \mathrm{H}), 5.79$ (ddd, $J=16.5,11.2,7.8 \mathrm{~Hz}, 1 \mathrm{H}), 5.07(\mathrm{~d}, J=0.7 \mathrm{~Hz}, 1 \mathrm{H}), 5.05-5.03(\mathrm{~m}$, 
1H), $4.47(\mathrm{~d}, J=11.6 \mathrm{~Hz}, 1 \mathrm{H}), 4.42(\mathrm{~d}, J=11.6 \mathrm{~Hz}, 1 \mathrm{H}), 4.11$ (brddd, $J=11.9,5.6,5.2 \mathrm{~Hz}, 1 \mathrm{H}$ ), 3.80 (s, 3H), 3.70 (ddd, $J=10.3,5.4,2.1 \mathrm{~Hz}, 1 \mathrm{H}), 3.45$ (dd, $J=9.4,5.6 \mathrm{~Hz}, 1 \mathrm{H}), 3.42(\mathrm{dd}, J=9.4$, $6.6 \mathrm{~Hz}, 1 \mathrm{H}$ ), 2.19 (brddd, $J=13.7,6.9,6.7 \mathrm{~Hz}, 2 \mathrm{H}), 1.67$ (ddd, $J=14.4,5.4,2.2 \mathrm{~Hz}, 1 \mathrm{H}), 1.60$ (ddd, $J=14.4,10.3,4.1 \mathrm{~Hz}, 1 \mathrm{H}), 1.01$ (d, $J=6.8 \mathrm{~Hz}, 3 \mathrm{H}$ ), 0.87 (s, 9H), 0.07 (s, 3H), 0.005 (s, $3 \mathrm{H}) ;{ }^{13} \mathrm{C}$ NMR $\left(125 \mathrm{MHz}, \mathrm{CDCl}_{3}\right) \square 159.1,140.5,130.1,129.2,115.2,113.6,73.1,72.9,71.3$, $70.1,55.2,44.1,37.6,25.8,17.9,15.7,-4.6,-5.0 ;\left(\mathrm{ES}^{+}\right) \mathrm{m} / \mathrm{z} 417.243003\left[(\mathrm{M}+\mathrm{Na})^{+}\right.$; calcd for $\left.\mathrm{C}_{22} \mathrm{H}_{38} \mathrm{NaO}_{4} \mathrm{Si}: 417.243708\right]$

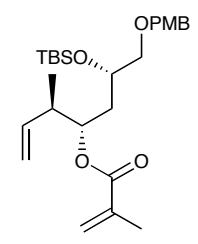

$(-)-S 19$

Diene (-)-S19. To a stirred solution of (-)-27 (0.750 g, $1.9 \mathrm{mmol})$ in dry $\mathrm{CH}_{2} \mathrm{Cl}_{2}(20 \mathrm{~mL})$, a catalytic amount of DMAP ( $15 \mathrm{mg})$ was added at room temperature. The reaction mixture was cooled to $78^{\circ} \mathrm{C} . \mathrm{Et}_{3} \mathrm{~N}(0.53 \mathrm{~mL}, 3.8 \mathrm{mmol})$ was added dropwise, immediately followed by acryloyl chloride $(0.278 \mathrm{~mL}, 2.85 \mathrm{mmol})$. After $2 \mathrm{~h}$ of stirring at $-78^{\circ} \mathrm{C}$, the reaction mixture was diluted with $\mathrm{CH}_{2} \mathrm{Cl}_{2}$ $(10 \mathrm{~mL})$, quenched with brine $(20 \mathrm{~mL})$ and warmed rapidly to $\mathrm{rt}$. The aqueous layer was extracted with $\mathrm{CH}_{2} \mathrm{Cl}_{2}(3 \times 20 \mathrm{~mL})$. The organic layer was dried over $\mathrm{MgSO}_{4}$, filtered and concentrated in vacuo. Flash chromatography (5\% ethyl acetate/hexanes) provided diene (-)-S19 in $80 \%$ yield; $[\square]_{\mathrm{D}}^{23}-13.5\left(\mathrm{c} \mathrm{0.4}, \mathrm{CHCl}_{3}\right) ; \mathrm{IR}$ (film, $\left.\mathrm{CHCl}_{3}\right) 2959,2928,2855,1716,1513,1248,1171,1105,836$ $\mathrm{cm}^{-1} ;{ }^{1} \mathrm{H}$ NMR $\left(500 \mathrm{MHz}, \mathrm{CDCl}_{3}\right) \square 7.23(\mathrm{~d}, J=8.6 \mathrm{~Hz}, 2 \mathrm{H}), 6.86(\mathrm{~d}, J=8.6 \mathrm{~Hz}, 2 \mathrm{H}), 6.07$ (dd, $J=$ 1.5, $0.9 \mathrm{~Hz}, 1 \mathrm{H}$ ), 5.75 (ddd, $J=17.5,10.4,7.2 \mathrm{~Hz}, 1 \mathrm{H}$ ), 5.52 (ddd, $J=3.2,1.5,1.5 \mathrm{~Hz}, 1 \mathrm{H}$ ), $5.09-$ $5.03(\mathrm{~m}, 2 \mathrm{H}), 4.44(\mathrm{~d}, J=11.6 \mathrm{~Hz}, 1 \mathrm{H}), 4.41(\mathrm{~d}, J=11.6 \mathrm{~Hz}, 1 \mathrm{H}), 4.85-4.81(\mathrm{~m}, 2 \mathrm{H}), 4.18-3.80(\mathrm{~s}$, 3H), $3.36(\mathrm{dd}, J=9.7,5.4 \mathrm{~Hz}, 1 \mathrm{H}), 3.30(\mathrm{dd}, J=9.7,5.6 \mathrm{~Hz}, 1 \mathrm{H}), 2.58-2.52(\mathrm{~m}, 1 \mathrm{H}), 1.93(\mathrm{dd}, J=$ 1.3, $1.1 \mathrm{~Hz}, 3 \mathrm{H}), 1.80$ (ddd, $J=14.5,9.6,3.2 \mathrm{~Hz}, 1 \mathrm{H}), 1.60$ (ddd, $J=14.5,8.9,2.8 \mathrm{~Hz}, 1 \mathrm{H}$ ), 0.99 (d, J = 6.9 Hz, 3H), 0.87 (s, 9H), 0.02 (s, 3H), 0.005 (s, 3H); ${ }^{13} \mathrm{C}$ NMR (125 MHz, $\left.\mathrm{CDCl}_{3}\right) \square 166.8$, 
$159.0,139.2,136.7,129.1,124.8,115.4,113.5,74.7,74.2,72.8,68.4,55.1,41.4,36.1,25.8$, 18.2, 18.0, 14.7, -4.2, -5.0; $\left(\mathrm{ES}^{+}\right) \mathrm{m} / \mathrm{z} 485.271887$ [(M+Na $)^{+}$; calcd for $\mathrm{C}_{26} \mathrm{H}_{42} \mathrm{O}_{5} \mathrm{SiNa}$ : 485.269923]

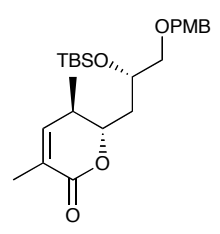

$(-)-28$

Lactone (-)-28. (4,5-DihydrolMES) $\left(\mathrm{PCy}_{3}\right) \mathrm{Cl}_{2} \mathrm{Ru}=\mathrm{CHPh}$ II $(0.059 \mathrm{~g}, 0.069 \mathrm{mmol})$ was added in one portion at room temperature to a stirred solution of (-)-S19 $(0.318 \mathrm{~g}, 0.688 \mathrm{mmol})$ in dry $\mathrm{CH}_{2} \mathrm{Cl}_{2}$ $(100 \mathrm{~mL})$. The reaction mixture was heated at reflux overnight (oil bath temperature: $58^{\circ} \mathrm{C}$ ), cooled to r.t., concentrated in vacuo and purified by flash chromatography (ethyl acetate/hexanes $30 \%)$. Lactone (-)-28 was obtained in $75 \%$ yield $(250 \mathrm{mg})$; $[\square]_{\mathrm{D}}^{23}-43.5\left(\mathrm{c} 0.22, \mathrm{CHCl}_{3}\right)$; IR (neat) 2954, 2927, 2855, 1722, 1513, 1248, 1128, 1097, $828 \mathrm{~cm}^{-1} ;{ }^{1} \mathrm{H}$ NMR $\left(500 \mathrm{MHz}, \mathrm{CDCl}_{3}\right) \square 7.24$ (d, $J=8.6 \mathrm{~Hz}, 2 \mathrm{H}), 6.87(\mathrm{~d}, J=8.6 \mathrm{~Hz}, 2 \mathrm{H}), 6.32(\mathrm{brs}, 1 \mathrm{H}), 4.46(\mathrm{~d}, J=11.8 \mathrm{~Hz}, 1 \mathrm{H}), 4.43(\mathrm{~d}, J=$ $11.8 \mathrm{~Hz}, 1 \mathrm{H}), 4.22-4.18(\mathrm{~m}, 1 \mathrm{H}), 4.18(\mathrm{ddd}, J=10.3,10.3,2.1 \mathrm{~Hz}, 1 \mathrm{H}), 3.80(\mathrm{~s}, 3 \mathrm{H}), 3.39(\mathrm{dd}, J=$ 9.8, $4.9 \mathrm{~Hz}, 1 \mathrm{H}), 3.34(\mathrm{dd}, J=9.8,5.3 \mathrm{~Hz}, 1 \mathrm{H}), 2.43-2.39(\mathrm{~m}, 1 \mathrm{H}), 1.89(\mathrm{brs}, 3 \mathrm{H}), 1.83$ (ddd, $J=$ 14.3, 10.4, $2.5 \mathrm{~Hz}, 1 \mathrm{H}$ ), 1.75 (ddd, $J=14.3,9.9,2.2 \mathrm{~Hz}, 1 \mathrm{H}$ ), 1.07 (d, J = 7.2 Hz, 3H), 0.86 (s, 9H), 0.05 (s, 6H); ${ }^{13} \mathrm{C}$ NMR (125 MHz, $\left.\mathrm{CDCl}_{3}\right) \square 165.3,159.1,145.7$, 133.3, 129.1, 127.1, 113.6, $79.9,74.5,72.8,66.9,55.2,38.1,34.0,25.8,18.0,16.7,16.6,-4.3,-4.9 ;\left(\mathrm{ES}^{+}\right) \mathrm{m} / \mathrm{z} 457.23903$ $\left[(\mathrm{M}+\mathrm{Na})^{+}\right.$; calcd for $\left.\mathrm{C}_{24} \mathrm{H}_{38} \mathrm{O}_{5} \mathrm{SiNa}: 457.238623\right]$.

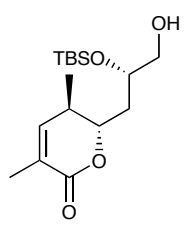

(-)-S20 
Alcohol (-)-S20: At $0{ }^{\circ} \mathrm{C}$, a solution of PMB ether (-)-28 (502 mg, $\left.1.18 \mathrm{mmol}\right)$ in $\mathrm{CH}_{2} \mathrm{Cl}_{2}(10 \mathrm{~mL})$ was treated with $\mathrm{H}_{2} \mathrm{O}(0.20 \mathrm{~mL})$ and $\mathrm{DDQ}(322 \mathrm{mg}, 1.42 \mathrm{mmol})$ and stirred for $1 \mathrm{~h}$. The mixture was quenched with $20 \mathrm{~mL}$ of saturated $\mathrm{NaHCO}_{3}$, washed with $\mathrm{H}_{2} \mathrm{O}(2 \times 15 \mathrm{~mL})$ and separated. The aqueous layer was then extracted with $\mathrm{CH}_{2} \mathrm{Cl}_{2}(3 \times 20 \mathrm{~mL})$. The combined organic extracts were dried $\left(\mathrm{MgSO}_{4}\right)$, filtered and concentrated. Flash chromatography (30\% ethyl acetate / hexanes) afforded alcohol (-)-S20, $337 \mathrm{mg}, 89 \%$ yield; [D] $]_{\mathrm{D}}^{23}-57.0\left(c 5, \mathrm{CHCl}_{3}\right)$; IR (neat) 3446, 2955, 2929, 2889, 2857, 1719, 1461, 1388, 1252, 1219, 1146, 1120, 1081, 1010, 836, $777 \mathrm{~cm}^{-1}$; ${ }^{1} \mathrm{H}$ NMR $\left(500 \mathrm{MHz}, \mathrm{CDCl}_{3}\right) \square 6.32(\mathrm{ddd}, J=4.1,1.5,1.5 \mathrm{~Hz}, 1 \mathrm{H}), 4.11$ (ddd, $J=10.4,10.4,1.9$ $\mathrm{Hz}, 1 \mathrm{H}), 4.17-4.12(\mathrm{~m}, 1 \mathrm{H}), 3.65(\mathrm{ddd}, J=11.2,3.0,3.0 \mathrm{~Hz}, 1 \mathrm{H}), 3.46$ (ddd, $J=11.2,7.8,3.0 \mathrm{~Hz}$, 1H), $2.46-2.38(\mathrm{~m}, 1 \mathrm{H}), 1.96(\mathrm{ddd}, J=14.5,10.0,1.9 \mathrm{~Hz}, 1 \mathrm{H}), 1.90(\mathrm{dd}, J=2.2,1.5 \mathrm{~Hz}, 3 \mathrm{H}), 1.83$ (br s, $1 \mathrm{H}), 1.71$ (ddd, $J=14.5,10.0,1.5 \mathrm{~Hz}, 1 \mathrm{H}), 1.08(\mathrm{~d}, J=7.1 \mathrm{~Hz}, 3 \mathrm{H}), 0.88(\mathrm{~s}, 9 \mathrm{H}), 0.10$ (s, 3H) , 0.08 (s, 3H); ${ }^{13} \mathrm{C}$ NMR (125 MHz, $\left.\mathrm{CDCl}_{3}\right) \square 156.3,145.9,127.1,80.1,68.2,66.9,37.4,34.0$, 25.8, 17.9, 16.8, 16.6, -4.5, -4.8; $\left(\mathrm{ES}^{+}\right) \mathrm{m} / \mathrm{z} 337.1808\left[(\mathrm{M}+\mathrm{Na})^{+}\right.$; calcd for $\mathrm{C}_{16} \mathrm{H}_{30} \mathrm{O}_{4} \mathrm{SiNa}$ : 337.1811].

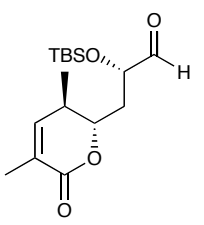

$(-)-18$

Aldehyde (-)-18: To a $0{ }^{\circ} \mathrm{C}$ solution of alcohol (-)-S20 (306 mg, $\left.0.974 \mathrm{mmol}\right)$ in $\mathrm{CH}_{2} \mathrm{Cl}_{2}(10 \mathrm{~mL})$ were added Dess-Martin periodinane $(823 \mathrm{mg}, 1.94 \mathrm{mmol})$ and $\mathrm{NaHCO}_{3}(352 \mathrm{mg}, 4.86 \mathrm{mmol})$. The resulting solution was stirred for $45 \mathrm{~min}$. and quenched with saturated $\mathrm{Na}_{2} \mathrm{~S}_{2} \mathrm{O}_{3}$ solution (25 $\mathrm{mL})$ and saturated $\mathrm{NaHCO}_{3}$ solution $(25 \mathrm{~mL})$. The mixture was then extracted with $\mathrm{Et}_{2} \mathrm{O}(4 \times 25$ $\mathrm{mL}$ ) and separated. The organic solution was then washed with $\mathrm{H}_{2} \mathrm{O}$, dried $\left(\mathrm{MgSO}_{4}\right)$, filtered, and concentrated. Flash chromatography (20\% ethyl acetate/hexanes) yielded aldehyde (-)-18 as a colorless oil (222 mg, 85\%). [प] $]_{\mathrm{D}}^{23}-8.0\left(\mathrm{c} 0.25, \mathrm{CHCl}_{3}\right)$; IR (neat) 2956, 2929, 2885, 2857, 1724, 
1472, 1254, 1140, 838, $779 \mathrm{~cm}^{-1} ;{ }^{1} \mathrm{H}$ NMR $\left(500 \mathrm{MHz}, \mathrm{CDCl}_{3}\right) \square 9.64(\mathrm{dd}, J=0.74,0.74 \mathrm{~Hz}, 1 \mathrm{H})$, $6.34(\mathrm{dd}, J=2.2,1.5 \mathrm{~Hz}, 1 \mathrm{H}), 4.42(\mathrm{dd}, J=10.4,2.6 \mathrm{~Hz}, 1 \mathrm{H}), 4.15$ (ddd, $J=10.4,10.4,2.2 \mathrm{~Hz}$, 1H), 2.52-2.43 (m, 1H), 2.01 (ddd, $J=14.5,10.8,2.6 \mathrm{~Hz}, 1 \mathrm{H}), 1.90(\mathrm{dd}, J=2.2,1.5 \mathrm{~Hz}, 3 \mathrm{H}), 1.78$ (ddd, $J=14.1,10.8,2.2 \mathrm{~Hz}, 1 \mathrm{H}), 1.08$ (d, $J=7.1 \mathrm{~Hz}, 3 \mathrm{H}$ ), 0.90 (s, 9H), 0.11 (s, 3H) , 0.08 (s, 3H); ${ }^{13} \mathrm{C}$ NMR $\left(125 \mathrm{MHz}, \mathrm{CDCl}_{3}\right) \square 202.7,164.7,145.6,127.2,78.7,73.5,35.4,33.9,25.7,18.0,16.7$, 16.4, -4.6, -5.2; $\left(\mathrm{ES}^{+}\right) \mathrm{m} / \mathrm{z} 335.1666\left[(\mathrm{M}+\mathrm{Na})^{+}\right.$; calcd for $\left.\mathrm{C}_{16} \mathrm{H}_{28} \mathrm{O}_{4} \mathrm{SiNa}: 335.1655\right]$.

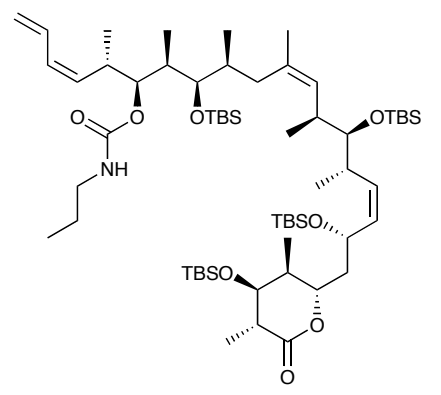

(+)-S21

n-Propyl carbamate (+)-S21: A solution of alcohol (+)-29 (11.0 mg, $0.011 \mathrm{mmol})$ in $\mathrm{CH}_{2} \mathrm{Cl}_{2}(0.83$ $\mathrm{mL}$ ) was treated with $n$-propyl isocyanate $(35 \mu \mathrm{L}, 0.33 \mathrm{mmol})$ and $\mathrm{DBU}(25 \mu \mathrm{L}, 0.16 \mathrm{mmol})$ at room temperature for $4 \mathrm{hr}$. The reaction mixture was quenched with saturated $\mathrm{NH}_{4} \mathrm{Cl}(2 \mathrm{~mL})$, and diluted with $\mathrm{CH}_{2} \mathrm{Cl}_{2}(5 \mathrm{~mL})$. The resulting layers were separated, and the aqueous layer was extracted with $\mathrm{CH}_{2} \mathrm{Cl}_{2}(3 \times 5 \mathrm{~mL})$. The combined organic layers were washed with saturated brine $(1 \times 8 \mathrm{~mL})$, dried over $\mathrm{Na}_{2} \mathrm{SO}_{4}$, filtered, and concentrated. Flash chromotography (5\% ethyl acetate/ hexanes) provided $10.0 \mathrm{mg}$ tetra-TBS carbamate (+)-S21 (83\%) as a colorless oil. $[\square]_{\mathrm{D}}^{23}$ +40.6 (c 1.0, $\mathrm{CHCl}_{3}$ ); IR (film, NaCl) 3404, 2958, 2930, 2889, 2861, 1734, 1700, 1457, 1253, 1123, 1071, 1045, 835, $774 \mathrm{~cm}^{-1} ;{ }^{1} \mathrm{H}$ NMR $\left(500 \mathrm{MHz}, \mathrm{CDCl}_{3}\right) \square 6.60$ (dddd, $J=16.8,11.2,10.4$, $1.1 \mathrm{~Hz}, 1 \mathrm{H}), 6.01(\mathrm{dd}, J=10.8,10.8 \mathrm{~Hz}, 1 \mathrm{H}), 5.38(\mathrm{dd}, J=10.0,10.0 \mathrm{~Hz}, 1 \mathrm{H}), 5.31-5.18(\mathrm{~m}, 3 \mathrm{H})$, $5.13(\mathrm{~d}, J=10.0 \mathrm{~Hz}, 1 \mathrm{H}), 5.04(\mathrm{~d}, J=10.0 \mathrm{~Hz}, 1 \mathrm{H}), 4.78(\mathrm{dd}, J=9.7,9.7 \mathrm{~Hz}, 1 \mathrm{H}), 4.75-4.71(\mathrm{~m}$, 1H), 4.59-4.54 (m, 1H), 4.52 (ddd, $J=10.8,10.8,1.1 \mathrm{~Hz}, 1 \mathrm{H}), 3.64$ (dd, $J=2.6,2.6 \mathrm{~Hz}, 1 \mathrm{H}), 3.45$ (dd, $J=4.5,4.5 \mathrm{~Hz}, 1 \mathrm{H}), 3.24(\mathrm{dd}, J=5.2,5.2 \mathrm{~Hz}, 1 \mathrm{H}), 3.18-3.05(\mathrm{~m}, 2 \mathrm{H}), 3.04-2.94(\mathrm{~m}, 1 \mathrm{H})$, 
2.65-2.55 (m, $2 \mathrm{H}), 2.50-2.42(\mathrm{~m}, 1 \mathrm{H}), 2.10(\mathrm{dd}, J=12.6,12.6 \mathrm{~Hz}, 1 \mathrm{H}), 1.94-1.78(\mathrm{~m}, 3 \mathrm{H}), 1.76-$ $1.65(\mathrm{~m}, 2 \mathrm{H}), 1.58(\mathrm{~s}, 3 \mathrm{H}), 1.56-1.52(\mathrm{~m}, 1 \mathrm{H}), 1.47$ (ddddd, $J=7.1,7.1,7.1,7.1,7.1 \mathrm{~Hz}, 2 \mathrm{H}), 1.23$ (d, $J=7.4 \mathrm{~Hz}, 3 \mathrm{H}), 0.99(\mathrm{~d}, J=6.7 \mathrm{~Hz}, 3 \mathrm{H}), 0.97(\mathrm{~d}, J=6.7 \mathrm{~Hz}, 3 \mathrm{H}), 0.96(\mathrm{~d}, J=7.1 \mathrm{~Hz}, 3 \mathrm{H})$, 0.93 (s, 9H), 0.92 (d, J=7.1 Hz, 3H), 0.91 (d, $J=7.1 \mathrm{~Hz}, 3 \mathrm{H}$ ), 0.90 (s, 9H), 0.88 (s, 9H), 0.87 (d, $J=6.7 \mathrm{~Hz}, 3 \mathrm{H}), 0.87(\mathrm{~s}, 9 \mathrm{H}), 0.72(\mathrm{~d}, J=6.7 \mathrm{~Hz}, 3 \mathrm{H}), 0.10(\mathrm{~s}, 3 \mathrm{H}), 0.08(\mathrm{~s}, 3 \mathrm{H}), 0.074(\mathrm{~s}, 3 \mathrm{H})$, $0.072(\mathrm{~s}, 3 \mathrm{H}), 0.062$ (s, 3H), 0.058 (s, 3H), 0.048 (s, 3H), 0.00 (s, 3H); ${ }^{13} \mathrm{C}$ NMR $(125 \mathrm{MHz}$, $\left.\mathrm{CDCl}_{3}\right) \square 173.2,156.8,133.8,137.5,132.4,132.2,131.8,131.4,129.6,117.8,80.5,78.3,77.2$, $76.9,74.9,64.6,44.2,42.72,42.67,37.9,37.5,35.9,35.47,35.44,35.2,34.6,34.2,26.3,26.2$, $25.9,25.7,23.2,23.0,18.5,18.4,18.09,17.96,17.5,17.1,16.4,14.1,13.8,11.1,10.1,-3.03,-$ $3.45,-3.53,-4.35,-4.37,-4.5,-4.84,-4.86$; high resolution mass spectrum $\left(\mathrm{ES}^{+}\right)$) $\mathrm{m} / \mathrm{z} 1114.7751$ $\left[(\mathrm{M}+\mathrm{Na})^{+}\right.$; calcd for $\mathrm{C}_{60} \mathrm{H}_{117} \mathrm{NO}_{8} \mathrm{Si}_{4} \mathrm{Na:}$ 1114.7754].

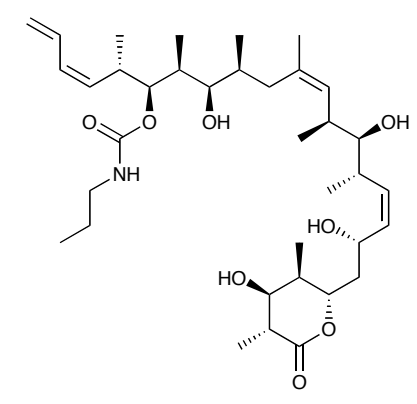

$(+)-30$

n-Propyl carbamatel tetra-ol (+)-30: Tetra-TBS carbamate (+)-S21 (10 mg, $9.2 \mu \mathrm{mol})$ was dissolved in $\mathrm{MeOH}(3.0 \mathrm{~mL})$. Aqueous $\mathrm{HCl}(6 \mathrm{~N}, 3.1 \mathrm{~mL})$ was added dropwise over $4 \mathrm{hr}$ at room temperature. The reaction mixture was stirred for $4 \mathrm{hr}$ further, diluted with water $(6 \mathrm{~mL})$ and EtOAc $(10 \mathrm{~mL})$. The resulting mixture was neutralized by solid $\mathrm{NaHCO}_{3}$, and separated. The aqueous layer was extracted with EtOAc $(3 \times 8 \mathrm{~mL})$. The combined organic layers were washed with saturated brine $(1 \times 15 \mathrm{~mL})$, dried over $\mathrm{Na}_{2} \mathrm{SO}_{4}$, filtered, and concentrated. Flash chromatography $\left(9 \% \mathrm{MeOH} / \mathrm{CH}_{2} \mathrm{Cl}_{2}\right)$ provided $n$-propyl carbamoyl tetra-ol (+)-30 (3.0 mg, 48\%) as a colorless oil. $[\square]_{D}^{23}+22.0(c 0.25, \mathrm{MeOH})$; IR (film, NaCl) 3401, 2966, 2934, 2875, 1699, $1520,1457,1379,1241,1101,1033,973,908,804 \mathrm{~cm}^{-1} ;{ }^{1} \mathrm{H} \mathrm{NMR}\left(500 \mathrm{MHz}, \mathrm{CD}_{3} \mathrm{OD}\right) \square 6.68$ 
(ddd, $J=16.8,10.4,10.4 \mathrm{~Hz}, 1 \mathrm{H}), 6.10(\mathrm{dd}, J=11.2,11.2 \mathrm{~Hz}, 1 \mathrm{H}), 5.60(\mathrm{dd}, J=10.8,10.8 \mathrm{~Hz}$, 1H), $5.46(\mathrm{dd}, J=10.8,10.8 \mathrm{~Hz}, 1 \mathrm{H}), 5.38(\mathrm{dd}, J=10.8,8.9 \mathrm{~Hz}, 1 \mathrm{H}), 5.27(\mathrm{~d}, J=16.8 \mathrm{~Hz}, 1 \mathrm{H})$, $5.16(\mathrm{~d}, J=10.1 \mathrm{~Hz}, 1 \mathrm{H}), 4.98(\mathrm{~d}, J=9.7 \mathrm{~Hz}, 1 \mathrm{H}), 4.84(\mathrm{dd}, J=7.8,4.1 \mathrm{~Hz}, 1 \mathrm{H}), 4.63-4.56(\mathrm{~m}$, 2H), $3.64(\mathrm{dd}, J=3.7,3.7 \mathrm{~Hz}, 1 \mathrm{H}), 3.22(\mathrm{dd}, J=7.8,3.7 \mathrm{~Hz}, 1 \mathrm{H}), 3.18-3.13(\mathrm{~m}, 1 \mathrm{H}), 3.12(\mathrm{dd}, J=$ 8.2, 3.3 Hz, 1H), 3.08-3.05 (m, 1H), 3.04 (dd, J = 6.7, $6.7 \mathrm{~Hz}, 1 \mathrm{H}), 2.73-2.66(\mathrm{~m}, 1 \mathrm{H}), 2.64$ (dddd, $J=7.4,7.4,7.4,4.5 \mathrm{~Hz}, 1 \mathrm{H}), 2.35$ (dddd, $J=16.4,7.8,6.7,6.7 \mathrm{~Hz}, 1 \mathrm{H}), 2.00-1.73(\mathrm{~m}, 5 \mathrm{H}), 1.61$ (br s, 3H), 1.57-1.46 (m, 3H), 1.30-1.26 (m, 1H), $1.25(\mathrm{~d}, J=7.4 \mathrm{~Hz}, 3 \mathrm{H}), 1.07$ (d, J=7.1 Hz, 3H), $1.06(\mathrm{~d}, J=7.1 \mathrm{~Hz}, 3 \mathrm{H}), 0.99(\mathrm{~d}, J=6.7 \mathrm{~Hz}, 3 \mathrm{H}), 0.94(\mathrm{~d}, J=6.7 \mathrm{~Hz}, 3 \mathrm{H}), 0.93-0.87(\mathrm{~m}, 6 \mathrm{H}), 0.79$ $(\mathrm{d}, J=6.3 \mathrm{~Hz}, 3 \mathrm{H}) ;{ }^{13} \mathrm{C}$ NMR $\left(125 \mathrm{MHz}, \mathrm{CD}_{3} \mathrm{OD}\right) \mathrm{T} 175.3,158.1,132.8,132.7,132.4,132.1$, $131.9,130.1,129.5,117.2,79.2,78.5,77.1,75.2,72.2,62.3,43.1,42.1,41.2,37.5,36.2,35.39$ (2), 35.35, 33.7, 33.1, 22.7, 22.0, 18.1, 16.7, 16.5, 14.6, 14.1, 11.8, 10.1, 8.1; high resolution mass spectrum $\left(\mathrm{ES}^{+}\right) \mathrm{m} / \mathrm{z} 658.4327$ [(M+Na) ${ }^{+}$; calcd for $\left.\mathrm{C}_{36} \mathrm{H}_{61} \mathrm{NO}_{8} \mathrm{Na:} 658.4295\right]$.

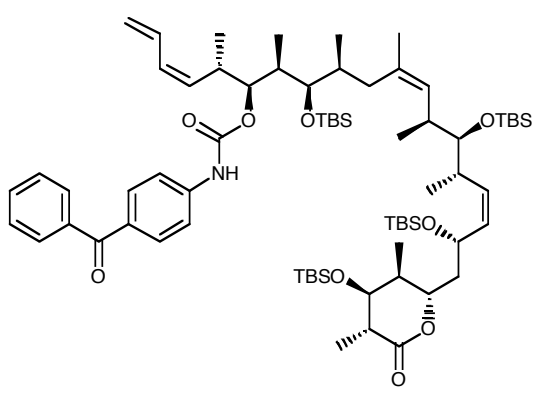

(+)-S22

Benzophenone carbamate (+)-S22. In a resealable Schlenk flask a suspension of 4-benzoyl-4benzoic acid $(100 \mathrm{mg}, 0.44 \mathrm{mmol})$ in benzene $(2.0 \mathrm{~mL})$ was treated with triethylamine $(0.10 \mathrm{~mL}$, $0.70 \mathrm{mmol}$ ) at $\mathrm{rt}$ to furnish a clear solution. To this solution was added subsequently diphenylphosphoryl azide $(0.11 \mathrm{~mL}, d=1.28,0.51 \mathrm{mmol})$ and the flask was sealed and heated to $80{ }^{\circ} \mathrm{C}$ for $3.0 \mathrm{~h}$ to afford a dark-yellow solution of benzophenone isocyanate (ca $0.2 \mathrm{M}$ in benzene). To a separate Schlenk flask charged with alcohol (+)-29 $(5.0 \mathrm{mg}, 0.49 \mathrm{mmol})$ was 
added a $0.4 \mathrm{~mL}$ aliquot of the previous isocyanate solution. The flask was sealed and heated to $45{ }^{\circ} \mathrm{C}$ for $5.0 \mathrm{~h}$. The reaction mixture was then directly applied as a benzene solution to a Merck preparative TLC plate $(0.25 \mathrm{~mm})$ and eluted $(9: 1$ hexanes/ EtOAc). The corresponding product band was cut and the resulting silica rinsed with EtOAc $(10 \mathrm{~mL})$ to furnish carbamate (+)-S22 (4.2 mg $70 \%$ ) as a colorless oil. $[\square]_{\mathrm{D}}^{23}+37\left(c\right.$ 0.11, $\left.\mathrm{CHCl}_{3}\right)$; IR (film, $\left.\mathrm{NaCl}\right) 33162957,2930,1734$ (broad), 1654, 1594, 1528, 1473, 1252, 1218, 1217, 1046, 836, $776 \mathrm{~cm}^{-1} ;{ }^{1} \mathrm{H}$ NMR $(500 \mathrm{MHz}$, $\left.\mathrm{CDCl}_{3}\right) \square 7.82(\mathrm{~d}, J=8.6 \mathrm{~Hz}, 2 \mathrm{H}), 7.78(\mathrm{~d}, J=8.5 \mathrm{~Hz}, 2 \mathrm{H}), 7.57($ app t, $J=7.6 \mathrm{~Hz}, 1 \mathrm{H}), 7.50-7.40$ (m, 4H), 6.74 (br s, 1H), 6.62 (ddd, $J=16.8,10.7,10.8 \mathrm{~Hz}, 1 \mathrm{H}), 6.10($ app t, $J=11.1 \mathrm{~Hz}, 1 \mathrm{H}$ ), $5.40(\operatorname{app~t}, J=10.7 \mathrm{~Hz}, 1 \mathrm{H}), 5.31-5.18(\mathrm{~m}, 5 \mathrm{H}), 5.08(\mathrm{~d}, J=9.0 \mathrm{~Hz}, 1 \mathrm{H}), 4.85(\operatorname{app~t}, J=6.0 \mathrm{~Hz}$, 1H), $4.79(\operatorname{app~t}, J=8.9 \mathrm{~Hz}, 1 \mathrm{H}), 4.50(\operatorname{app~t}, J=10.3 \mathrm{~Hz}, 1 \mathrm{H}), 3.61(\mathrm{br} \mathrm{s}, 1 \mathrm{H}), 3.49(\operatorname{app~t}, J=$ 4.1 Hz, 1H), 3.25 (app t, $J=5.0 \mathrm{~Hz}, 1 \mathrm{H}$ ), $3.05(\operatorname{app~q,~} J=16.0 \mathrm{~Hz}, 1 \mathrm{H}), 2.63-2.57(\mathrm{~m}, 2 \mathrm{H}), 2.49-$ $2.41(\mathrm{~m}, 1 \mathrm{H}), 2.11(\operatorname{app~t}, J=11.0 \mathrm{~Hz}, 1 \mathrm{H}), 2.02-1.97(\mathrm{~m}, 1 \mathrm{H}), 1.90-1.82(\mathrm{~m}, 1 \mathrm{H}), 1.80($ app t, $J=$ $9.2 \mathrm{~Hz}, 1 \mathrm{H}), 1.72-1.65(\mathrm{~m}, 2 \mathrm{H}), 1.60(\mathrm{~s}, 3 \mathrm{H}), 1.21(\mathrm{~d}, J=7.6 \mathrm{~Hz}, 3 \mathrm{H}), 1.05(\mathrm{~d}, J=6.8 \mathrm{~Hz}, 3 \mathrm{H})$, 1.00 (d, $J=7.2 \mathrm{~Hz}, 3 \mathrm{H}), 0.99$ (d, $J=7.2 \mathrm{~Hz}, 3 \mathrm{H}), 0.98-0.94(\mathrm{~m}, 3 \mathrm{H}), 0.97$ (d, J = 6.8 Hz, 3H), 0.94 (s, 9H), $0.90(\mathrm{~s}, 9 \mathrm{H}), 0.88(\mathrm{~s}, 9 \mathrm{H}), 0.85(\mathrm{~s}, 9 \mathrm{H}), 0.79(\mathrm{~d}, J=6.8 \mathrm{~Hz}, 3 \mathrm{H}), 0.11(\mathrm{~s}, 3 \mathrm{H}), 0.09(\mathrm{~s}, 3 \mathrm{H})$, 0.08-0.01 (m, 15H), $-0.005(\mathrm{~s}, 3 \mathrm{H}) ;{ }^{13} \mathrm{C}$ NMR (125 MHz, $\left.\mathrm{CDCl}_{3}\right) \square$ 195.4, 173.2, 153.1, 142.1, $138.0,133.4,133.2,132.4,132.1,132.0,131.97,131.67,131.2,130.1,129.7,128.2,128.1$ $118.3,117.4,80.4,79.8,77.1,76.6,74.8,64.6,44.1,42.6,37.6,37.4,35.8,35.4,35.2,34.4$, $34.2,26.3,26.2,25.8,25.6,25.0,23.0,18.5,18.4,18.0,17.9,17.4,17.2,16.4,16.1,13.7,10.2$, 3.4, -3.6, -4.2, -4.44, -4.42, -4.6, -4.92, -4.94; HRMS $\left(\mathrm{ES}^{+}\right) \mathrm{m} / \mathrm{z} 1252.7860\left[(\mathrm{M}+\mathrm{Na})^{+}\right.$; calc'd for $\left.\mathrm{C}_{70} \mathrm{H}_{119} \mathrm{NOgNaSi}_{4}: 1252.7838\right]$. 


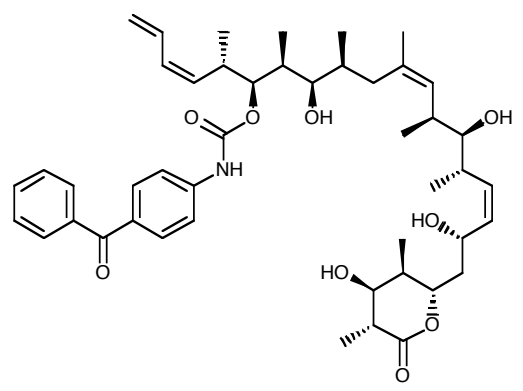

$(+)-31$

Benzophenone carbamate tetra-ol (+)-31. Warning: the following procedure was performed in a minimum of ambient light due to the photosensitivity of the final product. A Teflon vessel was charged with a solution of carbamate (+)-S22 $(4.5 \mathrm{mg}, 0.0037 \mathrm{mmol})$ in $\mathrm{CH}_{3} \mathrm{CN}(1.0 \mathrm{~mL})$ and stirred for $15 \mathrm{~min}$ at $0{ }^{\circ} \mathrm{C}$. To this solution was added aqueous hydrofluoric acid $(0.05 \mathrm{~mL}, 50 \%)$. Over the next $4.0 \mathrm{~h}$ a solution of hydrochloric acid $(3 \mathrm{~N}, 2.0 \mathrm{~mL})$ was added dropwise at a rate which minimized precipitation of the substrate (15 min intervals). This reaction mixture was maintained in absolute darkness for a total of $18 \mathrm{~h}$. At this time the solution was quenched with solid $\mathrm{NaHCO}_{3}$ (70 mg, portionwise to prevent overflow) and diluted with $40 \mathrm{~mL}$ of EtOAc. The resulting biphasic mixture was separated and the aqueous layer was further extracted with $3 \times 10$ $\mathrm{mL}$ portions of EtOAc. The combined organics were dried $\left(\mathrm{Na}_{2} \mathrm{SO}_{4}\right)$, filtered, and concentrated. Preparative TLC (Merck-0.25 mm plate, 95:5 $\mathrm{CH}_{2} \mathrm{Cl}_{2} / \mathrm{MeOH}$ elution and extraction) furnished (+)$31(2.1 \mathrm{mg}, 74 \%$ yield $) \cdot[\square]_{\mathrm{D}}^{23}+9.0\left(c 0.1, \mathrm{C}_{6} \mathrm{H}_{6}\right)$; IR (film, NaCl) 3320, 2963, 2923, 1725, 1713, 1643, 1597, 1530, 1315, 1222, $1024 \mathrm{~cm}^{-1} ;{ }^{1} \mathrm{H}$ NMR (500 MHz, CD $\left.3 \mathrm{CN}\right) \square 8.12(\mathrm{br} \mathrm{s}, 1 \mathrm{H}), 7.78$ (app dd, $J=8.0,2.1 \mathrm{~Hz}, 2 \mathrm{H}), 7.75(\operatorname{app~d}, J=9.0,2 \mathrm{H}), 7.64(\operatorname{app~t}, J=7.6 \mathrm{~Hz}, 1 \mathrm{H}), 7.55(\mathrm{~d}, J=$ $9.0 \mathrm{~Hz}, 2 \mathrm{H}), 7.51($ app t, $J=8.0 \mathrm{~Hz}, 2 \mathrm{H}), 6.72(\mathrm{ddd}, J=16.5,10.8,10.6 \mathrm{~Hz}, 1 \mathrm{H}), 6.08$ (app t, $J=$ $11.0 \mathrm{~Hz}, 1 \mathrm{H}), 5.55($ app t, $J=11.0 \mathrm{~Hz}, 1 \mathrm{H}), 5.47($ app t, $J=10.9 \mathrm{~Hz}, 1 \mathrm{H}) 5.37$ (dd, $J=16.0,2.0$ $\mathrm{Hz}, 1 \mathrm{H}), 5.25(\operatorname{app~d}, J=10.0 \mathrm{~Hz}, 1 \mathrm{H}), 5.18(\mathrm{~d}, J=10.3 \mathrm{~Hz}, 1 \mathrm{H}), 5.03(\mathrm{~d}, J=10.4 \mathrm{~Hz}, 1 \mathrm{H}), 4.87$ (app t, $J=6.0 \mathrm{~Hz}, 1 \mathrm{H}), 4.52-4.42(\mathrm{~m}, 2 \mathrm{H}), 3.63-3.49(\mathrm{~m}, 2 \mathrm{H}), 3.30(\mathrm{~m}, 1 \mathrm{H}), 3.25(\operatorname{app~d}, J=11.0$, 1H), $3.15(\mathrm{~m}, 1 \mathrm{H}), 3.07(\mathrm{~m}, 1 \mathrm{H}) 2.78(\mathrm{app} \mathrm{t}, J=4.4 \mathrm{~Hz}, 1 \mathrm{H}), 2.69-2.65(\mathrm{~m}, 3 \mathrm{H}), 2.56(\mathrm{dd}, J=13.0$, $7.0 \mathrm{~Hz}, 1 \mathrm{H}), 2.34(\mathrm{~m}, 1 \mathrm{H}), 1.93-1.88(\mathrm{~m}, 4 \mathrm{H}), 1.74(\mathrm{app} \mathrm{t}, J=13.0 \mathrm{~Hz}, 1 \mathrm{H}), 1.58(\mathrm{~s}, 3 \mathrm{H}), 1.52$, 
(dd, $J=14.3,10.6 \mathrm{~Hz}, 1 \mathrm{H}), 1.18(\mathrm{~d}, J=8.0 \mathrm{~Hz}, 3 \mathrm{H}), 1.00(\mathrm{~d}, J=7.3 \mathrm{~Hz}, 3 \mathrm{H}), 0.98(\mathrm{~d}, J=7.0 \mathrm{~Hz}$, 3H), 0.97 (d, J=7.2 Hz, 3H), $0.95(\mathrm{~d}, J=7.1 \mathrm{~Hz}, 3 \mathrm{H}), 0.90$ (d, $J=7.1 \mathrm{~Hz}, 3 \mathrm{H}), 0.86(\mathrm{~d}, J=7.2$ $\mathrm{Hz}, 3 \mathrm{H}) ;{ }^{13} \mathrm{C}$ NMR $\left(125 \mathrm{MHz}, \mathrm{CD}_{3} \mathrm{CN}\right) \square 196.0,173.7,153.8,143.0,138.1,137.0,135.7,133.1$, $132.8,132.7,132.2,131.9,131.34,131.33,130.2,129.7,129.4,128.2,118.7,79.2,78.7,76.7$ $74.9,72.1,65.3,43.0,41.2,38.9,37.3,36.0,35.5,35.3,33.8,33.1,22.3,18.5,17.0,16.4,14.7$, 14.0, 12.0, 8.5; $\mathrm{HRMS}\left(\mathrm{ES}^{+}\right) \mathrm{m} / z$ 796.4400 [(M+Na) ${ }^{+}$; calc'd for $\left.\mathrm{C}_{46} \mathrm{H}_{63} \mathrm{NO}_{9} \mathrm{Na}: 796.4433\right]$.

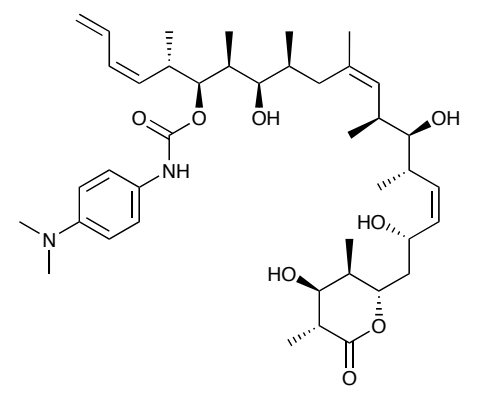

$(+)-32$

Dimethylaniline carbamate triol (+)-32. A solution of alcohol (+)-29 $(63 \mathrm{mg}, 0.067 \mathrm{mmol})$ in pyridine $(3.0 \mathrm{~mL})$ was treated with 4-dimethylaminophenyl isocyanate $(55 \mathrm{mg}, 0.336 \mathrm{mmol})$ at room temperature for $24 \mathrm{hr}$. The reaction mixture was quenched with saturated $\mathrm{NH}_{4} \mathrm{Cl}(2 \mathrm{~mL})$, and diluted with $\mathrm{CH}_{2} \mathrm{Cl}_{2}(5 \mathrm{~mL})$. The resulting layers were separated, and the aqueous layer was extracted with $\mathrm{CH}_{2} \mathrm{Cl}_{2}(3 \times 5 \mathrm{~mL})$. The combined organic layers were washed with saturated brine $(1 \times 8 \mathrm{~mL})$, dried over $\mathrm{Na}_{2} \mathrm{SO}_{4}$, filtered, and concentrated. Flash chromotography $(5 \%$ to $10 \%$ ethyl acetate/ hexanes) provided $42 \mathrm{mg}$ of dimethylaniline carbamate (+)-S23 (57\%) as a colorless oil which was directly subjected to the deprotection conditions.

Carbamate (+)-S23. (10 mg, $9.0 \mu \mathrm{mol})$ was dissolved in $\mathrm{MeOH}(1 \mathrm{~mL})$. Aqueous $\mathrm{HCl}(4 \mathrm{~N}, 4 \mathrm{~mL})$ was added dropwise over $2 \mathrm{~h}$ at room temperature. The reaction mixture was stirred for $4 \mathrm{hr}$ further, diluted with water $(10 \mathrm{~mL})$ and EtOAc $(25 \mathrm{~mL})$. The resulting mixture was neutralized by solid $\mathrm{NaHCO}_{3}$, and separated. The aqueous layer was extracted with EtOAc $(3 \times 8 \mathrm{~mL})$. The combined organic layers were washed with saturated brine $(1 \times 15 \mathrm{~mL})$, dried over $\mathrm{Na}_{2} \mathrm{SO}_{4}$, filtered, and concentrated. Flash chromotography (2\% to $\left.5 \% \mathrm{MeOH} / \mathrm{CH}_{2} \mathrm{Cl}_{2}\right)$ provided 
dimethylaniline carbamate triol $(+)-32(4.0 \mathrm{mg}, 62 \%)$ as a colorless solid. $[\square]_{D}^{23}+14.9$ (c 0.175 , $\mathrm{MeOH}$ ); IR (film, NaCl) 3393, 2965, 1704, 1524, $1234 \mathrm{~cm}^{-1} ;{ }^{1} \mathrm{H}$ NMR (500 MHz, $\left.\mathrm{CD}_{3} \mathrm{OD}\right) \square 7.24$ (d, $J=6.4 \mathrm{~Hz}, 2 \mathrm{H}), 6.76(\mathrm{~d}, J=6.4 \mathrm{~Hz}, 2 \mathrm{H}), 6.74(\mathrm{ddd}, J=16.9,10.7,10.7 \mathrm{~Hz}, 1 \mathrm{H}), 6.08(\operatorname{app~t}, J=$ $11.0 \mathrm{~Hz}, 1 \mathrm{H}), 5.62(\operatorname{app~t}, J=10.3 \mathrm{~Hz}, 1 \mathrm{H}), 5.50(\mathrm{~m}, 1 \mathrm{H}), 5.38(\mathrm{dd}, J=8.8,10.8 \mathrm{~Hz}, 1 \mathrm{H}), 5.27$ (dd, $J=1.6,16.7 \mathrm{~Hz}, 1 \mathrm{H}), 5.16(\mathrm{~d}, J=10.3 \mathrm{~Hz}, 1 \mathrm{H}), 4.96(\mathrm{~d}, J=10.1 \mathrm{~Hz}, 1 \mathrm{H}), 4.90(\mathrm{br} \mathrm{s}, 1 \mathrm{H})$, $4.58(\operatorname{app~t}, J=10.5 \mathrm{~Hz}, 2 \mathrm{H}), 3.54(\operatorname{app~t}, J=3.8 \mathrm{~Hz}, 1 \mathrm{H}), 3.25(\mathrm{~m}, 1 \mathrm{H}), 3.19(\mathrm{~m}, 1 \mathrm{H}), 3.12(\mathrm{dd}, J$ = 3.1, 8.5 Hz, 1H), $2.86(\mathrm{~s}, 6 \mathrm{H}), 2.69(\mathrm{~m}, 1 \mathrm{H}), 2.61(\mathrm{~m}, 1 \mathrm{H}), 2.35(\mathrm{~m}, 1 \mathrm{H}), 1.92($ app t, $J=10.2$ $\mathrm{Hz}, 1 \mathrm{H}), 1.87-1.72(\mathrm{~m}, 6 \mathrm{H}), 1.61(\mathrm{~s}, 3 \mathrm{H}), 1.50(\mathrm{~m}, 1 \mathrm{H}), 1.20(\mathrm{~d}, J=7.4 \mathrm{~Hz}, 3 \mathrm{H}), 1.08(\mathrm{~d}, J=7.4$ Hz, 3H), 1.03 (d, J = 7.4 Hz, 3H), 1.00 (d, J=7.4 Hz, 3H), 0.97 (d, J=7.4 Hz, 3H), 0.95 (d, J = $7.4 \mathrm{~Hz}, 3 \mathrm{H}), 0.79$ (d, J = 7.4 Hz, 3H); ${ }^{13} \mathrm{C}$ NMR $\left(125 \mathrm{MHz}, \mathrm{CD}_{3} \mathrm{OD}\right) \square 175.4,147.4,132.7,132.5$, $132.4,132.2,132.1,131.9,130.1,129.6,120.4,117.3,113.6$, (one quaternary aromatic signal was significantly braodened and could not be resolved), 79.2, 78.4, 77.0, 75.3, 72.2, 62.3, 43.1, $41.0,40.2,37.5,36.3,35.6,35.3,35.2,33.7,32.8,22.0,18.2,16.74,16.72,14.6,13.7,11.8,8.3$; high resolution mass spectrum $\left(\mathrm{ES}^{+}\right) \mathrm{m} / \mathrm{z}$ 735.4533. [(M+Na) ${ }^{+}$; calcd for $\mathrm{C}_{41} \mathrm{H}_{64} \mathrm{~N}_{2} \mathrm{O}_{8} \mathrm{Na}$ : 735.4560 .

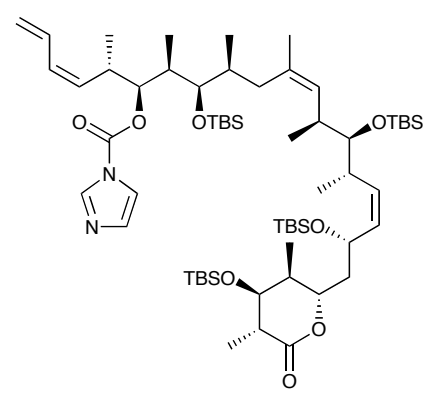

(+)-S24

Tetra-TBS carbonyl imidazole (+)-S24. To a solution of alcohol (+)-19 (45 mg, $0.0479 \mathrm{mmol})$ in $\mathrm{CH}_{2} \mathrm{Cl}_{2}(1.2 \mathrm{~mL})$ in a sealable reaction vessel was added carbonyl diimidazole (38 $\mathrm{mg}, 0.239$ $\mathrm{mmol})$. The vessel was sealed, and the mixture heated to $40^{\circ} \mathrm{C}$ and stirred for $16 \mathrm{~h}$. Solvent was removed and the residue subjected to rapid flash chromatography ( $20 \%$ ethyl acetate/hexanes) to provide tetra-TBS carbonyl imidazole (+)-S24 (32 $\mathrm{mg})$ which was used directly in the proceeding carbamate formation reactions. 


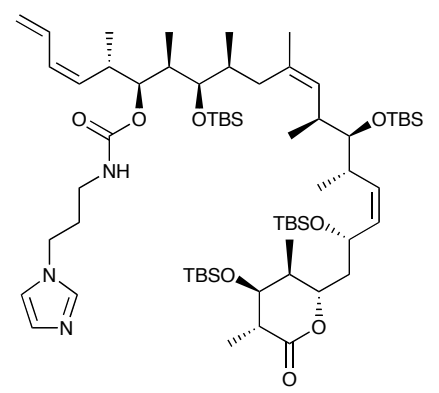

S25

Propylimidazole carbamate S25. A solution of the tetra-TBS carbonyl imidazole (+)-S24 (20 mg, $0.018 \mathrm{mmol})$ was dissolved in acetonitrile $(0.5 \mathrm{~mL})$. Methyl iodide $(0.35 \mathrm{~mL}, 5.6 \mathrm{mmol})$ was added and the solution was heated to $60{ }^{\circ} \mathrm{C}$ for $2 \mathrm{~h}$. The volatiles were removed by evaporation and the residue was redissolved in methylene chloride $(0.5 \mathrm{~mL}), 1$-(3-aminopropyl)-imidazole $(0.050 \mathrm{~mL}, 0.42 \mathrm{mmol})$ was added, and the resulting reaction mixture was stirred overnight. The volatiles were removed by evaporation and the crude residue was purified by flash chromatography (95:5 methylene chloride, methanol) to give propylimidazole carbamate S25 (16 mg, 77\%) ${ }^{1} \mathrm{H}$ NMR (400 MHz, $\left.\mathrm{CDCl}_{3}\right) \square 7.48(\mathrm{~s}, 1 \mathrm{H}), 7.06$ (s, 1H), $6.92(\mathrm{~s}, 1 \mathrm{H}), 6.60$ (ddd, $J=$ 16.7, 10.4, 10.4 Hz, 1H), 6.00 (dd, $J=10.8,10.8 \mathrm{~Hz}, 1 \mathrm{H}), 5.36(\mathrm{dd}, J=10.4,10.4 \mathrm{~Hz}, 1 \mathrm{H}), 5.32-$ $5.11(\mathrm{~m}, 4 \mathrm{H}), 5.05(\mathrm{~d}, J=9.8 \mathrm{~Hz}, 1 \mathrm{H}), 4.78(\mathrm{dd}, J=9.4,9.4 \mathrm{~Hz}, 1 \mathrm{H}), 4.70$ (ddd, $J=17.0,6.0,6.0$ $\mathrm{Hz}, 1 \mathrm{H}), 4.51(\mathrm{dd}, J=10.4,10.4 \mathrm{~Hz}, 1 \mathrm{H}), 3.97(\mathrm{dd}, J=7.0,7.0 \mathrm{~Hz}, 2 \mathrm{H}), 3.66-3.30(\mathrm{~m}, 1 \mathrm{H}), 3.42$ (dd, $J=4.3,4.3 \mathrm{~Hz}, 1 \mathrm{H}), 3.22(\mathrm{dd}, J=5.7,5.7 \mathrm{~Hz}, 2 \mathrm{H}), 3.13-3.03(\mathrm{~m}, 1 \mathrm{H}), 3.02-2.96(\mathrm{~m}, 1 \mathrm{H})$, 2.61-2.55 (m, 2H), 2.50-2.40 (m, 1H), $2.08(\mathrm{dd}, J=11.7,11.7 \mathrm{~Hz}, 1 \mathrm{H}), 2.01-1.62(\mathrm{~m}, 9 \mathrm{H}), 1.57(\mathrm{~s}$, 3H), $1.22(\mathrm{~d}, J=7.6 \mathrm{~Hz}, 3 \mathrm{H}), 0.98(\mathrm{~d}, J=6.7 \mathrm{~Hz}, 3 \mathrm{H}), 0.96(\mathrm{~d}, J=6.7 \mathrm{~Hz}, 3 \mathrm{H}), 0.96-0.81(\mathrm{~m}, 9 \mathrm{H})$, $0.92(\mathrm{~s}, 9 \mathrm{H}), 0.89(\mathrm{~s}, 9 \mathrm{H}), 0.87(\mathrm{~s}, 9 \mathrm{H}), 0.86(\mathrm{~s}, 9 \mathrm{H}), 0.71(\mathrm{~d}, J=7.1 \mathrm{~Hz}, 3 \mathrm{H}) 0.08(\mathrm{~s}, 3 \mathrm{H}), 0.06$ (app s, 9H), $0.044(\mathrm{~s}, 6 \mathrm{H}), 0.038(\mathrm{~s}, 3 \mathrm{H}),-0.01(\mathrm{~s}, 3 \mathrm{H}) ;{ }^{13} \mathrm{C}-\mathrm{NMR}\left(100 \mathrm{MHz}, \mathrm{CDCl}_{3}\right) \mathrm{T} 173.4$, $156.9,137.0,133.9,133.4,132.6,132.1,131.9,131.2,129.7$ (2), 118.8, 118.1, 80.4, 78.8, 74.8, $77.2,76.9,64.6,44.2(2), 42.6,37.9,37.5,37.4,35.7,35.4,35.1,34.5,34.0,31.6,26.3,26.2$, $25.9,25.7,23.1,18.5,18.4,18.1,17.9,17.5,17.1,16.5,16.1,14.1,13.8,10.1,-2.9,-3.4,-3.6,-$ $4.38,-4.42,-4.5,-4.9(2)$. 


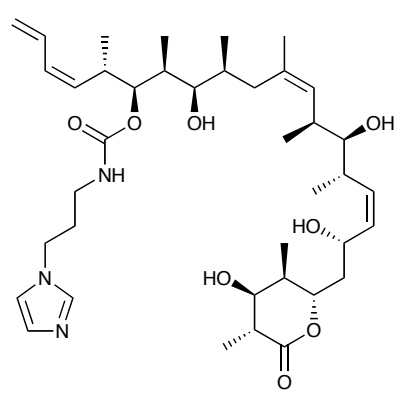

$(+)-33$

Propylimidazole carbamate tetra-ol (+)-33. The tetra-TBS propylimidazole carbamate (+)-S25 (16 $\mathrm{mg}, 0.014 \mathrm{mmol}$ ) was dissolved in methanol $(10 \mathrm{~mL})$ to which $3 \mathrm{~N} \mathrm{HCl}(10 \mathrm{~mL})$ was added in $2 \mathrm{~mL}$ aliquots and the resulting reaction mixture was stirred overnight. The reaction mixture was then diluted with water $(10 \mathrm{~mL})$ and EtOAc $(15 \mathrm{~mL})$, and the resulting mixture was neutralized with solid $\mathrm{NaHCO}_{3}$, and the layers separated. The aqueous layer was extracted with EtOAc $(3 \mathrm{x}$ $8 \mathrm{~mL})$. The combined organic layers were washed with saturated brine $(1 \times 15 \mathrm{~mL})$, dried over $\mathrm{Na}_{2} \mathrm{SO}_{4}$, filtered, and concentrated. Flash chromatography $\left(5 \% \mathrm{MeOH} / \mathrm{CH}_{2} \mathrm{Cl}_{2}\right)$ provided propylimidazole carbamate (+)-33 (6 mg, 62\%). $[\square]_{\mathrm{D}}^{23}+11.8\left(\right.$ c $\left.0.22, \mathrm{CH}_{2} \mathrm{Cl}_{2}\right) ; \mathrm{IR}$ (film, $\left.\mathrm{NaCl}\right)$ 3419, 2928, 1725, 1515, 1458, 1264, $1100 \mathrm{~cm}^{-1}$; ${ }^{1} \mathrm{H}-\mathrm{NMR}\left(400 \mathrm{MHz}, \mathrm{CDCl}_{3}\right) \square 7.54(\mathrm{~s}, 1 \mathrm{H}), 7.01$ (s, 1H), $6.91(\mathrm{~s}, 1 \mathrm{H}), 6.60$ (ddd $J=16.7,10.8,10.8 \mathrm{~Hz}, 1 \mathrm{H}$ ), 5.97 (dd, $J=10.8,10.8 \mathrm{~Hz}, 1 \mathrm{H}$ ), 5.50 (dd, $J=10.8,7.8 \mathrm{~Hz}, 1 \mathrm{H}), 5.46-5.05(\mathrm{~m}, 5 \mathrm{H}), 4.72-4.60(\mathrm{~m}, 2 \mathrm{H}), 3.96(\mathrm{dd}, J=5.6,5.6 \mathrm{~Hz}, 2 \mathrm{H})$, 3.69-3.57 (m, 1H), 3.23-3.15 (m, 2H), 3.15-2.78 (m, 6H), 2.76-2.61 (m, 3H), 2.06-1.77 (m, 7H), $1.64(\mathrm{~m}, 1 \mathrm{H}), 1.59(\mathrm{~s}, 3 \mathrm{H}), 1.29(\mathrm{~d}, J=7.8 \mathrm{~Hz}, 3 \mathrm{H}), 1.05(\mathrm{~d}, J=7.1 \mathrm{~Hz}, 3 \mathrm{H}), 1.02-0.91(\mathrm{~m}, 12 \mathrm{H})$, $0.82(\mathrm{~d}, J=6.3 \mathrm{~Hz}, 3 \mathrm{H}) ;{ }^{13} \mathrm{C}-\mathrm{NMR}\left(100 \mathrm{MHz}, \mathrm{CDCl}_{3}\right) \square 174.5,156.8,137.3,134.4,134.1,133.4$, 133.0, 132.3, 129.5, 129.4, 128.7, 118.7, 117.6, 79.0, 77.8, (1 obscured by $\mathrm{CDCl}_{3}$ ), 74.8, 72.8, $63.9,44.2,43.2,41.0,37.6,37.2,36.2,36.1,35.7,35.6,34.9,32.5,31.1,23.2,18.4,17.2,16.5$, 15.7, 13.4, 12.7, 9.3; HRMS $\left(\mathrm{ES}^{+}\right) \mathrm{m} / 2702.4688$ [(M+H) ${ }^{+}$; calc'd for $\mathrm{C}_{39} \mathrm{H}_{64} \mathrm{~N}_{3} \mathrm{O}_{8}:$ 702.4695]. 




S26

Propylmorpholine carbamate S26. A solution of the tetra-TBS carbonyl imidazole (+)-S24 (20 $\mathrm{mg}, 0.018 \mathrm{mmol})$ was dissolved in acetonitrile $(0.5 \mathrm{~mL})$. Methyl iodide $(0.35 \mathrm{~mL}, 5.6 \mathrm{mmol})$ was added and the solution was heated to $60{ }^{\circ} \mathrm{C}$ for $2 \mathrm{~h}$. The volatiles were removed by evaporation and the residue was redissolved in methylene chloride $(0.5 \mathrm{~mL})$ and 4-(3-aminopropyl)morpholine $(0.050 \mathrm{~mL}, 0.34 \mathrm{mmol})$ was added and the resulting reaction mixture was stirred overnight. The volatiles were removed by evaporation and the crude residue was purified by flash chromatography (95:5 methylene chloride/methanol) to give the propylmorpholine carbamate S26 (20 mg, 94\%). ${ }^{1} \mathrm{H}-\mathrm{NMR}\left(400 \mathrm{MHz}, \mathrm{CDCl}_{3}\right) \square 6.59$ (ddd, $\left.J=16.7,10.8,10.8 \mathrm{~Hz}, 1 \mathrm{H}\right), 6.00$ (dd, $J=$ 10.8, 10.8 Hz, 1H), 5.58 (dd, $J=5.6,5.6 \mathrm{~Hz}, 1 \mathrm{H}), 5.37$ (dd, $J=10.8,10.8 \mathrm{~Hz}, 1 \mathrm{H}), 5.35-4.99$ (m, $5 \mathrm{H}), 4.78(\mathrm{dd}, J=8.9,8.9 \mathrm{~Hz}, 1 \mathrm{H}), 4.51(\mathrm{dd}, J=10.8,10.8 \mathrm{~Hz}, 1 \mathrm{H}), 3.70(\mathrm{~m}, 4 \mathrm{H}), 3.63(\mathrm{dd}, J=$ 2.5, $2.5 \mathrm{~Hz}, 1 \mathrm{H}), 3.42(\mathrm{dd}, J=4.0,4.0 \mathrm{~Hz}, 1 \mathrm{H}), 3.32-3.20(\mathrm{~m}, 1 \mathrm{H}), 3.20-3.14(\mathrm{~m}, 2 \mathrm{H}), 3.04-2.92$ (m, 1H), 2.62 (dddd, $J=7.4,7.4,7.4,2.7 \mathrm{~Hz}, 1 \mathrm{H}), 2.62-2.57(\mathrm{~m}, 1 \mathrm{H}), 2.50-2.37(\mathrm{~m}, 6 \mathrm{H}), 2.07$ (dd, $J=12.9,12.9 \mathrm{~Hz}, 1 \mathrm{H}), 1.92-1.85(\mathrm{~m}, 3 \mathrm{H}), 1.77-1.50(\mathrm{~m}, 7 \mathrm{H}), 1.57(\mathrm{~s}, 3 \mathrm{H}), 1.22(\mathrm{~d}, J=7.0 \mathrm{~Hz}$ $3 \mathrm{H}), 1.01-0.83(\mathrm{~m}, 12 \mathrm{H}), 0.92(\mathrm{~s}, 9 \mathrm{H}), 0.89(\mathrm{~s}, 9 \mathrm{H}), 0.87-0.84(\mathrm{~d}$, obscured, $3 \mathrm{H}), 0.87(\mathrm{~s}, 9 \mathrm{H}), 0.86$ (s, 9H) $0.71(\mathrm{~d}, J=6.3 \mathrm{~Hz}, 3 \mathrm{H}), 0.09(\mathrm{~s}, 3 \mathrm{H}), 0.07$ (s, 6H), $0.06(\mathrm{~s}, 3 \mathrm{H}), 0.05(\mathrm{~s}, 6 \mathrm{H}), 0.04$ (s, 3H), $-0.01(\mathrm{~s}, 3 \mathrm{H}) ;{ }^{13} \mathrm{C}-\mathrm{NMR}\left(100 \mathrm{MHz}, \mathrm{CDCl}_{3}\right) \square 173.4,156.8,133.9,133.4,132.5,132.1,131.8$, 131.3, 129.6, 117.9, 80.4, 78.1, 76.9, (1 obscured by $\mathrm{CDCl}_{3}$ ), 74.9, 67.1, 64.6, 57.3, 53.7, 44.2, $42.6,40.4,37.7,37.4,35.7,35.5,35.1,34.6,34.0,29.7,26.3,26.2,25.9,25.7,23.0,18.5,18.4$, $18.1,17.9,17.6,17.1,16.5,16.2,14.2,13.9,10.0,-2.9,-3.4,-3.5,-4.37,-4.40,-4.5,-4.9(2)$. 


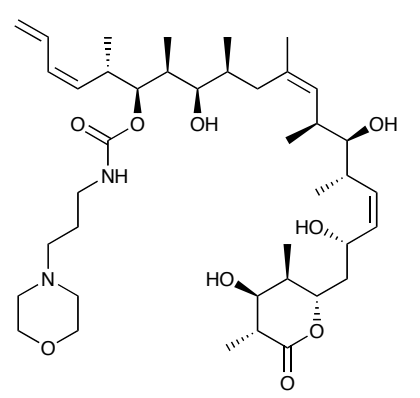

$(+)-34$

Propylmorpholine carbamate tetra-ol (+)-34. The tetra TBS propylmorpholine carbamate S26 (20 mg, $0.017 \mathrm{mmol}$ ) was dissolved in methanol $(10 \mathrm{~mL})$ to which $3 \mathrm{~N} \mathrm{HCl}(10 \mathrm{~mL})$ was added in $2 \mathrm{~mL}$ aliquots and the resulting reaction mixture was stirred overnight. The reaction mixture was then diluted with water $(10 \mathrm{~mL})$ and EtOAc $(15 \mathrm{~mL})$, and the resulting mixture was neutralized with solid $\mathrm{NaHCO}_{3}$, and the layers separated. The aqueous layer was extracted with EtOAc $(3 \mathrm{x}$ $10 \mathrm{~mL})$. The combined organic layers were washed with saturated brine $(1 \times 15 \mathrm{~mL})$, dried over $\mathrm{Na}_{2} \mathrm{SO}_{4}$, filtered, and concentrated. Flash chromatography $\left(5 \% \mathrm{MeOH} / \mathrm{CH}_{2} \mathrm{Cl}_{2}\right)$ provided propylmorpholine carbamate tetra-ol (+)-34 (8 mg, 65\%). [ []$_{\mathrm{D}}^{23}+20.6\left(c 0.3, \mathrm{CH}_{2} \mathrm{Cl}_{2}\right)$; IR (film, $\mathrm{NaCl}) 3417,2966,1702,1518,1457,1261,1114 \mathrm{~cm}^{-1} ;{ }^{1} \mathrm{H}-\mathrm{NMR}\left(400 \mathrm{MHz}, \mathrm{CDCl}_{3}\right) \square 6.59$ (ddd, J $=16.7,10.8,10.8 \mathrm{~Hz}, 1 \mathrm{H}), 5.98(\mathrm{dd}, J=10.8,10.8 \mathrm{~Hz}, 1 \mathrm{H}), 5.75(\mathrm{dd}, J=4.5,4.5 \mathrm{~Hz}, 1 \mathrm{H}), 5.49$ (dd, $J=11.9,7.1 \mathrm{~Hz}, 1 \mathrm{H}), 5.34-5.38(\mathrm{~m}, 2 \mathrm{H}), 5.23-5.07(\mathrm{~m}, 3 \mathrm{H}), 4.69-4.73(\mathrm{~m}, 2 \mathrm{H}), 4.61(\mathrm{dd}, J=$ $10.4,10.4 \mathrm{~Hz}, 1 \mathrm{H}), 3.67-3.70(\mathrm{~m}, 5 \mathrm{H}), 3.25-3.20(\mathrm{~m}, 2 \mathrm{H}), 3.20-3.15(\mathrm{~m}, 3 \mathrm{H}), 3.00-2.78(\mathrm{~m}, 1 \mathrm{H})$, 2.80-2.70 (m, $1 \mathrm{H}), 2.70-2.61(\mathrm{~m}, 2 \mathrm{H}), 2.60-2.48(\mathrm{~m}, 2 \mathrm{H}), 2.48-2.32(\mathrm{~m}, 7 \mathrm{H}), 1.95-1.81(\mathrm{~m}, 6 \mathrm{H})$, 1.64-1.55 (m, 6H), 1.28 (d, J=7.0 Hz, 3H), 1.05 (d, J=7.0 Hz, 3H), 0.99 (d, J = 7.0 Hz, 3H), 0.96 $(\mathrm{d}, J=6.7 \mathrm{~Hz}, 3 \mathrm{H}), 0.93(\mathrm{~d}, J=7.0 \mathrm{~Hz}, 3 \mathrm{H}), 0.92(\mathrm{~d}, J=7.0 \mathrm{~Hz}, 3 \mathrm{H}) 0.81(\mathrm{~d}, J=6.3 \mathrm{~Hz}, 3 \mathrm{H}) ;{ }^{13} \mathrm{C}-$ NMR $\left(100 \mathrm{MHz}, \mathrm{CDCl}_{3}\right) \square 174.5,156.8,134.0,133.9,133.0,132.9,132.1,129.8,129.6,117.8$, $78.9,78.2,77.2,76.1,72.9,66.9,64.0,57.0,53.5,43.2,41.0,40.1,37.2,36.2,35.8,35.4,35.1$,

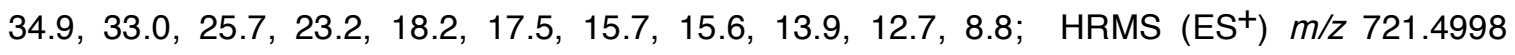
$\left[(\mathrm{M}+\mathrm{H})^{+}\right.$; calc'd for $\left.\mathrm{C}_{40} \mathrm{H}_{69} \mathrm{~N}_{2} \mathrm{O}_{9}: 721.5020\right]$. 


\section{Synthesis of the Previously Reported (+)-14-Normethyldiscodermolide}

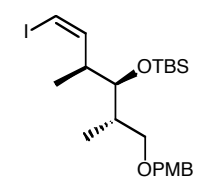

$(+)-\mathrm{N} 1$

Vinyl iodide (+)-N1: lodomethyltriphenylphosphonium iodide $(1.38 \mathrm{~g}, 2.61 \mathrm{mmol})$ was heated $\left(50^{\circ} \mathrm{C}\right)$ in vacuo for $12 \mathrm{~h}$, cooled, backfilled with $\mathrm{Ar}$, then supended in $6.0 \mathrm{~mL}$ of THF. $2.5 \mathrm{~mL}$ NaHMDS was added (1.0 M THF, $2.51 \mathrm{mmol}$ ) at rt and the mixture was stirred for 10 minutes. The red/brown suspension was cooled to $-60^{\circ} \mathrm{C}, 750 \mu \mathrm{L}$ of HMPA (freshly distilled) was added, and the orange suspension was cooled to $-78^{\circ} \mathrm{C}$. The substrate aldehyde N2 ${ }^{1}(795 \mathrm{mg})$ was added as a solution in THF (1.0 mL) via cannula and the cold bath was removed. After stirring for 30 minutes the brown suspension was diluted with hexanes and poured into $\mathrm{NH}_{4} \mathrm{Cl}$ solution. The organic layer was washed $\left(\mathrm{H}_{2} \mathrm{O}\right.$, brine), dried $\left(\mathrm{Na}_{2} \mathrm{SO}_{4}\right)$, filtered and concentrated. Flash chromatography (gradient elution: $15 \% \quad \mathrm{CH}_{2} \mathrm{Cl}_{2} / \mathrm{hexane}$ to $2 \% \mathrm{EtOAc/hexanes}$ to $5 \%$ EtOAc/hexane) furnished vinyl iodide (+)-N1 as a 7:1 Z/E mixture of olefin isomers (811 $\mathrm{mg}, 77 \%$ 2 steps). $[\square]_{\mathrm{D}}^{23}+27\left(\right.$ c $\left.0.60, \mathrm{CHCl}_{3}\right) ; \mathrm{IR}\left(\mathrm{CHCl}_{3}\right) 2928,2855,1512,1248,1078,837,774 \mathrm{~cm}^{-1}$; ${ }^{1} \mathrm{H}$ NMR $\left(500 \mathrm{MHz}, \mathrm{CDCl}_{3}\right): \square 7.26(\mathrm{~d}, J=8.3 \mathrm{~Hz}, 2 \mathrm{H}), 6.88(\mathrm{~d}, J=8.5 \mathrm{~Hz}, 2 \mathrm{H}), 6.09(\mathrm{~d}, J=7.3$ $\mathrm{Hz}, 1 \mathrm{H}), 6.07(\mathrm{dd}, J=8.7,7.4 \mathrm{~Hz}, 1 \mathrm{H}), 4.42(\mathrm{ABq}, J=11.6, \square \square=14.4 \mathrm{~Hz}, 2 \mathrm{H}), 3.81(\mathrm{~s}, 3 \mathrm{H}), 3.65$ (app t, $J=5.3 \mathrm{~Hz}, 1 \mathrm{H}), 3.52(\mathrm{dd}, J=9.15 .5 \mathrm{~Hz}, 1 \mathrm{H}), 3.26($ app t, $J=8.5 \mathrm{~Hz}, 1 \mathrm{H}), 2.70(\mathrm{dq}, J=$ 13.4, 6.6 Hz, 1H), $2.00(\mathrm{~m}, 1 \mathrm{H}), 1.01(\mathrm{~m}, 1 \mathrm{H}), 0.97(\mathrm{~d}, J=6.8 \mathrm{~Hz}, 3 \mathrm{H}), 0.89(\mathrm{~s}, 1 \mathrm{H}), 0.04(\mathrm{~s}, 3 \mathrm{H})$, $0.03(\mathrm{~s}, 3 \mathrm{H}) ;{ }^{13} \mathrm{C}$ NMR $\left(125 \mathrm{MHz}, \mathrm{CDCl}_{3}\right) \square 159.0,145.1,130.9,129.4,113.7,80.6,76.2,72.6$, $72.3,55.2,42.7,38.8,26.1,18.3,14.8,14.4,-3.8,-4.0$; high resolution mass spectrum $\left(\mathrm{ES}^{+}\right) \mathrm{m} / \mathrm{z}$ 527.1473 [(M+Na) ${ }^{+}$; calcd for $\left.\mathrm{C}_{22} \mathrm{H}_{37} \mathrm{O}_{3} \mathrm{SiNal}: 527.1454\right]$.

1 Structure 19, Smith, A. B., III; Beauchamp, T. J.; LaMarche, M. J.; Kaufman, M. D.; Qiu, Y.; Arimoto, H.; Jones, D. R.; Kobayashi, K. J. Am. Chem. Soc. 2000, 122 (36), 8654-8664. 


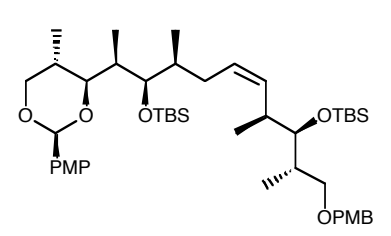

(+)-N3

PMP Acetal (+)-N3: A $1.0 \mathrm{M}$ solution of anhydrous $\mathrm{ZnCl}_{2}(1.10 \mathrm{~mL}, 1.10 \mathrm{mmol})$ was added via syringe to a solution of alkyl iodide $(+)-\mathrm{A}^{2}(602 \mathrm{mg}, 1.10 \mathrm{mmol})$ in dry $\mathrm{Et}_{2} \mathrm{O}(4.0 \mathrm{~mL})$, and the resulting solution was cooled to $-78{ }^{\circ} \mathrm{C}$ and degassed $2 \times$ (freeze-pump thaw). $t$-BuLi (1.7 $\mathrm{M}$ in pentane, $1.94 \mathrm{~mL}, 3.30 \mathrm{mmol}$ ) was added via cannula over $5 \mathrm{~min}$. The resultant solution was stirred 5 min further, evacuated ( $1 \square 0.1$ Torr) and back-filled with argon. The $-78{ }^{\circ} \mathrm{C}$ bath was removed, and the reaction was stirred at ambient temperature for $1 \mathrm{~h}$. The resulting cloudy suspension was transferred by cannula into an intimate mixture of vinyl iodide (+)-N1 (410 mg, $0.826 \mathrm{mmol} ; 7: 1 \mathrm{Z} / \mathrm{E})$ and $\mathrm{Pd}\left(\mathrm{PPh}_{3}\right)_{4}(48 \mathrm{mg}, 0.0413 \mathrm{mmol})$. The reaction mixture was stirred overnight in the absence of light, and quenched via slow addition of the reaction mixture to water $(200 \mathrm{~mL})$. The mixture was diluted with $\mathrm{Et}_{2} \mathrm{O}$, and the layers were separated. The water layer was extracted $\left(3 \square \mathrm{Et}_{2} \mathrm{O}\right)$, and the combined organic layers were washed (saturated aqueous $\mathrm{NaHCO}_{3}$, brine), dried $\left(\mathrm{MgSO}_{4}\right)$, filtered and concentrated. Flash chromatography (gradient elution: 2\% EtOAc/hexanes $\square$ 5\% EtOAc/hexanes) furnished (+)-N3 [397 mg, 60\% yield); [D] $]_{D}^{23}$ +34 (c 1.0, $\left.\mathrm{CHCl}_{3}\right) ; \mathrm{IR}\left(\mathrm{CHCl}_{3}\right)$ 2956, 2855, 1614, 1515, 1462, 1389, 1249, 1037, 835, $773 \mathrm{~cm}^{-1}$; ${ }^{1} \mathrm{H}$ NMR $\left(500 \mathrm{MHz}, \mathrm{CDCl}_{3}\right) \square 7.35(\mathrm{~d}, J=11.2 \mathrm{~Hz}, 2 \mathrm{H}), 7.21(\mathrm{~d}, J=11.6 \mathrm{~Hz}, 2 \mathrm{H}), 6.84($ app t, $J=$ $9.0 \mathrm{~Hz}, 4 \mathrm{H}), 5.37(\mathrm{~s}, 1 \mathrm{H}), 5.22(\mathrm{~m}, 2 \mathrm{H}), 4.35(\mathrm{~s}, 2 \mathrm{H}), 4.07(\mathrm{dd}, J=4.6,11.1 \mathrm{~Hz}, 1 \mathrm{H}), 3.77(\mathrm{~s}, 3 \mathrm{H})$, 3.76 (s, 3H), 3.65 (d, $J=7.3 \mathrm{~Hz}, 1 \mathrm{H}$ ), $3.45(\mathrm{~m}, 3 \mathrm{H}), 3.20$ (app t, $J=8.6 \mathrm{~Hz}, 1 \mathrm{H}$ ), 2.57 (app ddq, $J$ $=6.6,9.0,6.6 \mathrm{~Hz}, 1 \mathrm{H}), 2.06(\mathrm{~m}, 3 \mathrm{H}), 1.95(\operatorname{app~t}, J=4.8 \mathrm{~Hz}, 1 \mathrm{H}), 1.92(\operatorname{app~t}, J=4.4 \mathrm{~Hz}, 1 \mathrm{H})$, $1.88(\operatorname{app~t}, J=7.1 \mathrm{~Hz}, 1 \mathrm{H}), 1.74(\mathrm{~m}, 1 \mathrm{H}), 0.99(\mathrm{~d}, J=7.1 \mathrm{~Hz}, 3 \mathrm{H}), 0.93(\mathrm{~d}, J=6.9 \mathrm{~Hz}, 3 \mathrm{H}), 0.89$ (s, 9H), $0.89(\mathrm{~d}, J=6.5 \mathrm{~Hz}, 3 \mathrm{H}), 0.86(\mathrm{~s}, 9 \mathrm{H}), 0.81(\mathrm{~d}, J=6.7 \mathrm{~Hz}, 3 \mathrm{H}), 0.03(\mathrm{~m}, 12 \mathrm{H}) ;{ }^{13} \mathrm{C}$ NMR

2 See structure A, Smith, A. B., III; Beauchamp, T. J.; LaMarche, M. J.; Kaufman, M. D.; Qiu, Y.; Arimoto, H.; Jones, D. R.; Kobayashi, K. J. Am. Chem. Soc. 2000, 122 (36), 8654-8664. 
$\left(125 \mathrm{MHz}, \mathrm{CDCl}_{3}\right) \square 159.7,159.0,134.9,131.5,131.0,129.0,127.6,127.3,113.7,113.5,100.9$, $83.1,78.1,77.2,73.3,72.6,72.5,55.2,38.7,38.2,36.6,35.4,33.2,30.8,26.2,26.1,18.5,18.4$, $16.8,14.6,13.2,12.2,10.9,-3.6,-3.7,-3.8,-3.9$; high resolution mass spectrum $\left(\mathrm{ES}^{+}\right) \mathrm{m} / \mathrm{z}$ $821.5222\left[(\mathrm{M}+\mathrm{Na})^{+}\right.$; calcd for $\left.\mathrm{C}_{46} \mathrm{H}_{78} \mathrm{O}_{7} \mathrm{Si}_{2} \mathrm{Na}: 821.5184\right]$.

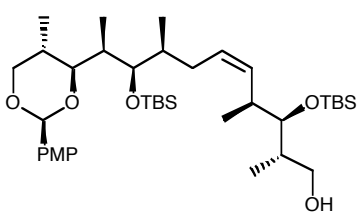

(+)-N4

Alcohol (+)-N4: At $0{ }^{\circ} \mathrm{C}$, a solution of PMB ether (+)-N3 (358 mg, $0.448 \mathrm{mmol}$ ) in $\mathrm{CH}_{2} \mathrm{Cl}_{2}(5 \mathrm{~mL})$ was treated with $\mathrm{H}_{2} \mathrm{O}(200 \mu \mathrm{L})$ and DDQ $(111 \mathrm{mg}, 0.490 \mathrm{mmol})$ and stirred for 60 min. The mixture was quenched with $20 \mathrm{~mL}$ of saturated $\mathrm{NaHCO}_{3}$, washed with $\mathrm{H}_{2} \mathrm{O}(4, \square)$ and separated. The aqueous layer was then extracted with $\mathrm{CH}_{2} \mathrm{Cl}_{2}(2 \mathrm{Z})$. The combined organic extracts were dried $\left(\mathrm{MgSO}_{4}\right)$, filtered, concentrated, dissolved in $\mathrm{MeOH}(20 \mathrm{~mL})$ and then treated with $\mathrm{NaBH}_{4}(2.24 \mathrm{mmol})$. The reaction mixture was concentrated, diluted with $\mathrm{CH}_{2} \mathrm{Cl}_{2}$, washed with aqueous saturated ammonium chloride and brine. The organic layer was dried over $\mathrm{NaSO}_{4}$, decanted, concentrated and chromatographed (10\% EtOAc/hexanes) to provide $244 \mathrm{mg}$ of (+)-N4 (80\%); [ []$_{\mathrm{D}}^{23}+41\left(c \mathrm{1.0}, \mathrm{CHCl}_{3}\right) ; \mathrm{IR}$ (film, NaCl) 3476, 2957, 2856, 1614, 1518, 1462, 1250, 1026, $835 \mathrm{~cm}^{-1} ;{ }^{1} \mathrm{H}$ NMR $\left(500 \mathrm{MHz}, \mathrm{CDCl}_{3}\right) \square 7.38(\mathrm{~d}, J=8.7 \mathrm{~Hz}, 2 \mathrm{H}), 6.87(\mathrm{~d}, J=8.7 \mathrm{~Hz}, 2 \mathrm{H})$, $5.38(\mathrm{~s}, 1 \mathrm{H}), 5.25(\mathrm{~m}, 2 \mathrm{H}), 4.08(\mathrm{dd}, J=11.2,4.7 \mathrm{~Hz}, 1 \mathrm{H}), 3.78(\mathrm{~s}, 3 \mathrm{H}), 3.69(\mathrm{dd}, J=7.3,1.6 \mathrm{~Hz}$, 1H), $3.65(\operatorname{appt}, J=5.1 \mathrm{~Hz}, 1 \mathrm{H}), 3.55(\operatorname{appt}, J=5.6 \mathrm{~Hz}, 1 \mathrm{H}), 3.52(\mathrm{~m}, 1 \mathrm{H}), 3.50(\operatorname{app~t}, J=11.4$, 1H), $3.46(\mathrm{dd}, J=6.2,4.3 \mathrm{~Hz}, 1 \mathrm{H}$ ), 2.67 (app ddq, $J=6.7,9.0,6.7 \mathrm{~Hz}, 1 \mathrm{H}), 2.34($ app t, $J=5.5$ $\mathrm{Hz}, 1 \mathrm{H}), 2.10(\mathrm{~m}, 2 \mathrm{H}), 1.98$ (ddd, $J=4.3,4.3,14 \mathrm{~Hz}, 1 \mathrm{H}), 1.87$ (ddd, $J=1.4,7.2,7.2 \mathrm{~Hz}, 1 \mathrm{H}$ ), $1.80(\mathrm{~m}, 1 \mathrm{H}), 1.78(\mathrm{~m}, 1 \mathrm{H}), 1.02(\mathrm{~d}, J=7.1 \mathrm{~Hz}, 3 \mathrm{H}), 0.99(\mathrm{~d}, J=7.1 \mathrm{~Hz}, 3 \mathrm{H}), 0.97(\mathrm{~d}, J=6.7 \mathrm{~Hz}$, 3H), $0.93(\mathrm{~s}, 9 \mathrm{H}), 0.92(\mathrm{~s}, 9 \mathrm{H}), 0.85(\mathrm{~d}, J=6.7 \mathrm{~Hz}, 3 \mathrm{H}), 0.70(\mathrm{~d}, J=7.1 \mathrm{~Hz}, 3 \mathrm{H}), 0.11(\mathrm{~s}, 3 \mathrm{H})$, 0.10 (s, 3H), 0.06 (s, 3H), 0.03 (s, 3H); $\left.{ }^{13} \mathrm{C} \mathrm{NMR} \mathrm{(125} \mathrm{MHz,} \mathrm{CDCl}_{3}\right) \square 159.7,133.9,131.5,128.4$, $127.3,113.4,100.9,83.0,81.2,76.7,73.3,65.3,55.2,38.4,38.2,36.8,36.5,33.2,30.7,26.2$, 
$26.1,18.5,18.3,17.3,15.7,13.3,12.1,10.9,-3.6,-3.7,-3.8(2)$; high resolution mass spectrum $\left(\mathrm{ES}^{+}\right) \mathrm{m} / \mathrm{z} 701.4582\left[(\mathrm{M}+\mathrm{Na})^{+}\right.$; calcd for $\left.\mathrm{C}_{38} \mathrm{H}_{70} \mathrm{O}_{6} \mathrm{NaSi}_{2}: 701.4609\right]$.

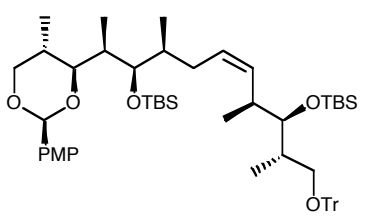

(+)-N5

Trityl protected acetal (+)-N5: To a solution of alcohol (+)-N4 (229 mg, $0.337 \mathrm{mmol})$ in pyridine (3.4 mL) was added trityl chloride (112.8 mg, $0.405 \mathrm{mmol})$ and DMAP (49.4 mg, $0.405 \mathrm{mmol})$. The mixture was then refluxed for $18 \mathrm{~h}$, cooled to ambient temperature, and added to a solution of $1 \mathrm{M}$ citric acid $(50 \mathrm{~mL})$. The mixture was extracted with $\mathrm{CH}_{2} \mathrm{Cl}_{2}$, washed with $1 \mathrm{M}$ citric acid, $\mathrm{H}_{2} \mathrm{O}$ $(100 \mathrm{~mL})$ and saturated $\mathrm{NaHCO}_{3}$ solution. The organic layers were separated, dried $\left(\mathrm{NaSO}_{4}\right)$, filtered, and concentrated in vacuo. Flash chromatography (5\% EtOAc/hexanes) provided (+)-N5 (271 mg, 87\%) as a white foam; $[\square]_{D}^{23}+36\left(c 1.0, \mathrm{CH}_{2} \mathrm{Cl}_{2}\right)$; IR $\left(\mathrm{CHCl}_{3}\right) 2929,2855,1615,1461$, 1250, 1036, 835, 773, $706 \mathrm{~cm}^{-1} ;{ }^{1} \mathrm{H}$ NMR (500 MHz, $\left.\mathrm{C}_{6} \mathrm{D}_{6}\right) \square 7.61(\mathrm{~d}, J=8.7 \mathrm{~Hz}, 2 \mathrm{H}), 7.59$ (app d, $J=7.4 \mathrm{~Hz}, 6 \mathrm{H}), 7.16(\mathrm{~m}, 6 \mathrm{H}), 7.05(\operatorname{app~t}, J=7.4 \mathrm{~Hz}, 3 \mathrm{H}), 6.83(\mathrm{~d}, J=8.7 \mathrm{~Hz}, 2 \mathrm{H}), 5.41(\mathrm{~s}, 1 \mathrm{H})$ 5.30 (app t, $J=10.3 \mathrm{~Hz}, 1 \mathrm{H}$ ), 5.25 (ddd, $J=10.9,9.2,4.1 \mathrm{~Hz}, 1 \mathrm{H}$ ), 3.96 (dd, $J=11.1,4.6 \mathrm{~Hz}$, 1H), $3.83(\mathrm{~d}, J=7.4 \mathrm{~Hz}, 1 \mathrm{H}), 3.65(\mathrm{dd}, J=6.8,4.0 \mathrm{~Hz}, 1 \mathrm{H}), 3.48(\mathrm{~m}, 2 \mathrm{H}), 3.28(\mathrm{~m}, 1 \mathrm{H}), 3.24(\mathrm{~s}$, 3H), 3.07 (app t, $J=8.8 \mathrm{~Hz}, 1 \mathrm{H}$ ), 2.77 (app ddq, $J=6.7,9.4,6.7 \mathrm{~Hz}, 1 \mathrm{H}$ ), $2.41(\mathrm{~m}, 1 \mathrm{H}$ ), 2.26 (ddd, $J=14.1,9.2,9.2 \mathrm{~Hz}, 1 \mathrm{H}), 1.93(\mathrm{~m}, 4 \mathrm{H}), 1.18(\operatorname{app~t}, J=6.8 \mathrm{~Hz}, 6 \mathrm{H}), 1.12(\mathrm{~d}, J=6.7 \mathrm{~Hz}$, 3H), $1.04(\mathrm{~d}, J=6.8 \mathrm{~Hz}, 3 \mathrm{H}), 1.03(\mathrm{~s}, 9 \mathrm{H}), 0.95(\mathrm{~s}, 9 \mathrm{H}), 0.91(\mathrm{~d}, J=6.7 \mathrm{~Hz}, 3 \mathrm{H}), 0.12(\mathrm{~s}, 3 \mathrm{H})$, 0.09 (s, 3H), 0.06 (s, 3H), 0.05 (s, 3H); ${ }^{13} \mathrm{C}$ NMR (125 MHz, $\left.\mathrm{C}_{6} \mathrm{D}_{6}\right) \square 160.4,145.1,134.8,132.3$, $129.2(2), 128.3,127.9,127.1,113.8,101.7,86.9,83.3,78.5,77.7,73.3,66.5,54.7,40.4,38.6$, $36.9,36.0,33.7,30.9,26.5,26.3,18.8,18.5,18.0,14.4,13.6,12.0,11.3,-3.3,-3.4,-3.7,-3.7$; high resolution mass spectrum $\left(\mathrm{ES}^{+}\right) \mathrm{m} / \mathrm{z} 943.5720\left[(\mathrm{M}+\mathrm{Na})^{+}\right.$; calcd for $\mathrm{C}_{57} \mathrm{H}_{84} \mathrm{O}_{6} \mathrm{Si}_{2} \mathrm{Na}$ : 943.5704]. 


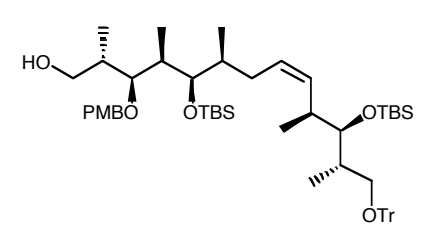

$(+)-\mathbf{N} 6$

Trityl protected alcohol (+)-N6: To a $0{ }^{\circ} \mathrm{C}$ solution of trityl ether (+)-N5 (267 $\left.\mathrm{mg}, 0.289 \mathrm{mmol}\right)$ in $\mathrm{CH}_{2} \mathrm{Cl}_{2}(4.0 \mathrm{~mL})$ was added DIBAl-H $(1 \mathrm{M}$ in Toluene, $0.87 \mathrm{~mL}, 0.868 \mathrm{mmol})$. The resulting solution was stirred for $4.5 \mathrm{~h}$, quenched via drop-wise addition of $\mathrm{pH} 7.0$ buffer $(20 \mathrm{~mL})$, then diluted with $\mathrm{CH}_{2} \mathrm{Cl}_{2}$. The mixture was then added to saturated sodium potassium tartrate solution, extracted with $\mathrm{CH}_{2} \mathrm{Cl}_{2}$, and separated. The organic layer was washed with $\mathrm{H}_{2} \mathrm{O}$, dried $\left(\mathrm{MgSO}_{4}\right)$, filtered, and concentrated. Flash chromatography (20\% EtOAc/hexanes) provided (+)N6 (257 mg, 96\%) as a white glass; $[\square]_{\mathrm{D}}^{23}+18\left(\right.$ ( 1.0, $\left.\mathrm{CHCl}_{3}\right)$; IR (film, $\left.\mathrm{NaCl}\right) 2930,2855,1613$, 1513, 1250, 1036, 836, 772, $706 \mathrm{~cm}^{-1} ;{ }^{1} \mathrm{H}$ NMR $\left(500 \mathrm{MHz}, \mathrm{CDCl}_{3}\right) \square 7.58(\operatorname{app~d}, J=8.5 \mathrm{~Hz}, 6 \mathrm{H})$, $7.28(\mathrm{~d}, J=8.6 \mathrm{~Hz}, 2 \mathrm{H}), 7.16(\mathrm{~m}, 6 \mathrm{H}), 7.05(\operatorname{app~t}, J=7.3 \mathrm{~Hz}, 3 \mathrm{H}), 6.80(\mathrm{~d}, J=8.5 \mathrm{~Hz}, 2 \mathrm{H}), 5.27$ (app t, $J=10.5 \mathrm{~Hz}, 1 \mathrm{H}), 5.14$ (ddd, $J=10.3,10.2,4.7 \mathrm{~Hz}, 1 \mathrm{H}), 4.53(\mathrm{ABq}, J=10.8, \square \square=13.1 \mathrm{~Hz}$, 2H), 3.70 (ddd, $J=10.1,5.1,4.8 \mathrm{~Hz}, 1 \mathrm{H}$ ), 3.65 (m, 2H), 3.55 (ddd, $J=10.4,5.1,4.9 \mathrm{~Hz}, 1 \mathrm{H}$ ), 3.46 (dd, $J=8.8,6.1 \mathrm{~Hz}, 1 \mathrm{H}), 3.40(\mathrm{dd}, J=6.5,4.2 \mathrm{~Hz}, 1 \mathrm{H}), 3.31(\mathrm{~s}, 3 \mathrm{H}), 3.06(\operatorname{app} \mathrm{t}, J=8.7 \mathrm{~Hz}, 1 \mathrm{H})$, 2.76 (app ddq, $J=6.7,9.4,6.7 \mathrm{~Hz}, 1 \mathrm{H}$ ), $2.38(\mathrm{~m}, 1 \mathrm{H}$ ), 2.16 (ddd, $J=14.1,9.8,9.8 \mathrm{~Hz}, 1 \mathrm{H}$ ), 2.04 (app t, $J=5.2 \mathrm{~Hz}, 1 \mathrm{H}), 1.94(\mathrm{~m}, 3 \mathrm{H}), 1.80(\mathrm{~m}, 1 \mathrm{H}), 1.16(\mathrm{~d}, J=6.8 \mathrm{~Hz}, 6 \mathrm{H}), 1.10(\mathrm{~d}, J=6.7 \mathrm{~Hz}$, 3H), $1.04(\mathrm{~s}, 9 \mathrm{H}), 0.95$ (d, J = 6.6 Hz, 6H), 0.95 (s, 9H), $0.14(\mathrm{~s}, 3 \mathrm{H}), 0.12(\mathrm{~s}, 3 \mathrm{H}), 0.09$ (s, 3H), 0.07 (s, 3H); ${ }^{13} \mathrm{C}$ NMR $\left(125 \mathrm{MHz}, \mathrm{CDCl}_{3}\right) \mathrm{P} 159.6,144.9,134.7,131.0,129.2,129.1,128.3$ (2), $126.9,114.0,86.7,84.6,78.0,76.9,74.9,66.4,65.1,54.6,40.3,39.9,38.3,37.9,35.7,32.9$, $26.4,26.2,18.6,18.3,17.8,15.3,14.1,13.8,11.6,-3.3,-3.4,-3.8,-3.9$; high resolution mass spectrum $\left(\mathrm{ES}^{+}\right) \mathrm{m} / \mathrm{z} 945.5843\left[(\mathrm{M}+\mathrm{Na})^{+}\right.$; calcd for $\left.\mathrm{C}_{57} \mathrm{H}_{86} \mathrm{O}_{6} \mathrm{Si}_{2} \mathrm{Na}: 945.5861\right]$.

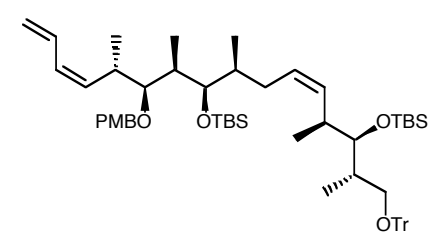


Trityl Protected Triene N7: To a $0{ }^{\circ} \mathrm{C}$ solution of alcohol (+)-N6 (225 mg, $\left.0.241 \mathrm{mmol}\right)$ in $\mathrm{CH}_{2} \mathrm{Cl}_{2}(3.0 \mathrm{~mL})$ were added Dess-Martin periodinane $(307 \mathrm{mg}, 0.724 \mathrm{mmol})$ and $\mathrm{NaHCO}_{3}(315$ $\mathrm{mg}, 4.34 \mathrm{mmol}$ ). The resulting solution was stirred for $2.5 \mathrm{~h}$ and quenched with saturated $\mathrm{NaS}_{2} \mathrm{O}_{3}$ solution and saturated $\mathrm{NaHCO}_{3}$ solution. The mixture was then extracted with $\mathrm{Et}_{2} \mathrm{O}(3$ x ) and separated. The organic solution was then washed with $\mathrm{H}_{2} \mathrm{O}$, dried $\left(\mathrm{MgSO}_{4}\right)$, filtered, and concentrated. The resulting white foam aldehyde N8 was used without further purification:

Aldehyde [N8]: ${ }^{1} \mathrm{H}$ NMR $\left(500 \mathrm{MHz}, \mathrm{C}_{6} \mathrm{D}_{6}\right) \mathrm{Q} 9.73(\mathrm{~d}, \mathrm{~J}=2.3 \mathrm{~Hz}, 1 \mathrm{H}), 7.59(\operatorname{app} \mathrm{d}, \mathrm{J}=$ $7.4 \mathrm{~Hz}, 6 \mathrm{H}), 7.22(\mathrm{~d}, \mathrm{~J}=8.6 \mathrm{~Hz}, 2 \mathrm{H}) 7.16(\mathrm{~m}, 6 \mathrm{H}), 7.06($ app t, J = 7.3 Hz, 3H), $6.79(\mathrm{~d}, \mathrm{~J}=8.6$ $\mathrm{Hz}, 2 \mathrm{H}), 5.28(\mathrm{app} \mathrm{t}, \mathrm{J}=10.5 \mathrm{~Hz}, 1 \mathrm{H}), 5.12(\mathrm{ddd}, \mathrm{J}=10.3,10.1,4.61 \mathrm{H}), 4.40(\mathrm{ABq}, \mathrm{J}=10.9, \square \square$ $=22.7 \mathrm{~Hz}, 2 \mathrm{H}), 3.66(\mathrm{~m}, 2 \mathrm{H}), 3.55(\operatorname{app~t}, \mathrm{J}=4.7 \mathrm{~Hz}, 1 \mathrm{H}), 3.47(\mathrm{dd}, \mathrm{J}=8.7,6.2 \mathrm{~Hz}, 1 \mathrm{H}), 3.31(\mathrm{~s}$, 3H), $3.06(a p p t, J=8.7 \mathrm{~Hz}, 1 \mathrm{H}), 2.74(\operatorname{app} d d q, J=6.7,9.5,6.7 \mathrm{~Hz}, 1 \mathrm{H}), 2.57(\mathrm{~m}, 1 \mathrm{H}), 2.39(\mathrm{~m}$, 1H), $2.13(\mathrm{ddd}, \mathrm{J}=14.1,9.7,9.7 \mathrm{~Hz}, 1 \mathrm{H}), 1.91(\mathrm{~m}, 2 \mathrm{H}), 1.74(\mathrm{~m}, 1 \mathrm{H}), 1.17(\mathrm{~d}, \mathrm{~J}=6.9 \mathrm{~Hz}, 3 \mathrm{H})$, $1.11(\mathrm{~d}, \mathrm{~J}=6.6 \mathrm{~Hz}, 3 \mathrm{H}), 1.10(\mathrm{~d}, \mathrm{~J}=6.9 \mathrm{~Hz}, 3 \mathrm{H}), 1.02(\mathrm{~s}, 9 \mathrm{H}), 0.95(\mathrm{~s}, 9 \mathrm{H}), 0.93$ (d, J = 7.0 Hz, 3H), $0.92(\mathrm{~d}, \mathrm{~J}=6.8 \mathrm{~Hz}, 3 \mathrm{H}), 0.13(\mathrm{~s}, 3 \mathrm{H}), 0.09(\mathrm{~s}, 3 \mathrm{H}), 0.07(\mathrm{~s}, 6 \mathrm{H}) ;{ }^{13} \mathrm{C} \mathrm{NMR}\left(125 \mathrm{MHz}, \mathrm{C}_{6} \mathrm{D}_{6}\right)$ ( $202.8,159.7,144.9,134.9,130.6,129.1,129.0,128.3,128.3,127.4,126.9,114.0,86.7,82.2$, $78.2,76.7,74.2,66.4,54.6,49.5,40.3,37.8,35.7,32.8,26.2,26.1,18.6,18.3,17.7,14.1,13.9$, $11.9,11.5,-3.4,-3.5,-3.8,-3.9$.

To a $-78{ }^{\circ} \mathrm{C}$ solution of freshly distilled allyldiphenylphosphine $(104 \mu \mathrm{L}, 0.482 \mathrm{mmol})$ in THF (1.0 mL, degassed) was added $283 \mu \mathrm{L}$ of $t$-butyllithium (1.7M in pentane, $0.482 \mathrm{mmol}$ ) and stirred for $5 \mathrm{~min}$. The solution was warmed to $0{ }^{\circ} \mathrm{C}$, stirred for $30 \mathrm{~min}$ and cooled to $-78{ }^{\circ} \mathrm{C}$. The solution was treated with freshly distilled $\mathrm{Ti}(i-\mathrm{OPr}) 4(142 \mu \mathrm{L}, 0.482 \mathrm{mmol})$ and stirred for $30 \mathrm{~min}$. A precooled $\left(-78{ }^{\circ} \mathrm{C}\right)$ solution of aldehyde $\mathrm{N8}(0.241 \mathrm{mmol})$ in THF $(1.0 \mathrm{~mL})$ was added via cannula (rinse $1 \times 1.0 \mathrm{~mL}$ ) and stirred for $1 \mathrm{~h}$, then warmed to $0{ }^{\circ} \mathrm{C}$. lodomethane $(225 \mu \mathrm{L}, 3.62$ mmol) was added, and the solution was warmed to ambient temperature and stirred for $16 \mathrm{~h}$. The solution was quenched with $\mathrm{pH} 7.0$ buffer and extracted with $\mathrm{CH}_{2} \mathrm{Cl}_{2}(3 \times 10 \mathrm{~mL})$ and $\mathrm{Et}_{2} \mathrm{O}(3 \mathrm{x}$ 
$10 \mathrm{~mL})$. The combined organic layers were washed with saturated brine solution, dried $\left(\mathrm{MgSO}_{4}\right)$, filtered, and concentrated. Flash chromatography (5\% EtOAc/hexanes) provided N7 (144 mg, $63 \%$ from (+)-N6, 10:1 mixture of diastereomers) as a white foam: IR $\left(\mathrm{CHCl}_{3}\right) 2956,2855,1613$ $\mathrm{cm}^{-1} ;{ }^{1} \mathrm{H}$ NMR (500 MHz, $\mathrm{C}_{6} \mathrm{D}_{6}$, major diastereomer) $7.60(\mathrm{~m}, 6 \mathrm{H}), 7.32(\mathrm{~d}, J=8.6 \mathrm{~Hz}, 2 \mathrm{H})$, $7.16(\mathrm{~m}, 6 \mathrm{H}), 7.06(\mathrm{~m}, 3 \mathrm{H}), 6.82(\mathrm{~d}, J=8.6 \mathrm{~Hz}, 2 \mathrm{H}) 6.70(\mathrm{ddd}, J=16.8,10.7,10.7 \mathrm{~Hz}, 1 \mathrm{H}) 6.05$ (app t, $J=11.0 \mathrm{~Hz}, 1 \mathrm{H}), 5.65(\operatorname{app~t}, J=10.5 \mathrm{~Hz}, 1 \mathrm{H}), 5.25(\operatorname{app~t}, J=10.2 \mathrm{~Hz}, 1 \mathrm{H}), 5.18(\mathrm{dd}, J=$ 16.7, $1.6 \mathrm{~Hz}, 1 \mathrm{H}), 5.10$ (ddd, $J=10.1,10.1,4.9 \mathrm{~Hz}, 1 \mathrm{H}), 5.07(\mathrm{~d}, J=10.3 \mathrm{~Hz}, 1 \mathrm{H}), 4.55(\mathrm{ABq}, J=$ 10.7, $\mathrm{QD}=48.6 \mathrm{~Hz}, 2 \mathrm{H}), 3.65(\mathrm{~m}, 2 \mathrm{H}), 3.46(\mathrm{dd}, J=8.8,6.1 \mathrm{~Hz}, 1 \mathrm{H}), 3.32(\mathrm{~s}, 3 \mathrm{H}), 3.06(\mathrm{~m}, 2 \mathrm{H})$, $2.71(\mathrm{~m}, 1 \mathrm{H}), 2.41(\mathrm{~m}, 1 \mathrm{H}), 1.97(\mathrm{~m}, 4 \mathrm{H}), 1.74(\mathrm{~m}, 1 \mathrm{H}), 1.20(\mathrm{~d}, J=6.9 \mathrm{~Hz}, 3 \mathrm{H}), 1.16(\mathrm{~d}, J=6.9$ $\mathrm{Hz}, 3 \mathrm{H}), 1.11(\mathrm{~d}, J=6.8 \mathrm{~Hz}, 3 \mathrm{H}), 1.09(\mathrm{~d}, J=6.7 \mathrm{~Hz}, 3 \mathrm{H}), 1.06(\mathrm{~s}, 9 \mathrm{H}), 0.98(\mathrm{~d}, J=6.8 \mathrm{~Hz}, 3 \mathrm{H})$, $0.95(\mathrm{~s}, 9 \mathrm{H}), 0.17(\mathrm{~s}, 3 \mathrm{H}), 0.13(\mathrm{~s}, 6 \mathrm{H}), 0.07(\mathrm{~s}, 3 \mathrm{H}) ;{ }^{13} \mathrm{C} \mathrm{NMR}\left(125 \mathrm{MHz}, \mathrm{C}_{6} \mathrm{D}_{6}\right.$, major diastereomer) $]$ 159.5, 144.9, 134.7, 134.5, 132.6, 131.4, 129.4, 129.2, 129.0, 128.3, 127.8, $126.9,117.5,113.9,86.7,84.6,78.2,76.9,75.1,66.4,54.6,40.4,40.3,38.3,35.8,35.6,32.3$, $26.4,26.2,18.7,18.6,18.3,17.7,15.1,14.0,11.1,-3.3,-3.4,-3.8,-3.9$; high resolution mass spectrum $\left(\mathrm{ES}^{+}\right) \mathrm{m} / \mathrm{z} 967.6086\left[(\mathrm{M}+\mathrm{Na})^{+}\right.$; calcd for $\left.\mathrm{C}_{60} \mathrm{H}_{88} \mathrm{O}_{5} \mathrm{Si}_{2} \mathrm{Na}: 967.6068\right]$.



(+)-N9

Triene Alcohol N10: Anhydrous $\mathrm{MeOH}$ (50 $\square \mathrm{L})$ was added to a cold $\left(0{ }^{\circ} \mathrm{C}\right)$ solution of chlorocatecholborane $(0.769 \mathrm{~g}, 4.97 \mathrm{mmol})$ in $1.5 \mathrm{~mL}$ of $\mathrm{CH}_{2} \mathrm{Cl}_{2}(3.2 \mathrm{M})$. The resulting solution was added in $48 \mu \mathrm{L}(0.153 \mathrm{mmol})$ aliquots at $10 \mathrm{~min}$ intervals to a $0.07 \mathrm{M}$ solution $\left(\mathrm{CH}_{2} \mathrm{Cl}_{2}\right)$ of trityl ether N7 (144 mg, $0.153 \mathrm{mmol}, 10: 1 \mathrm{dr})$ at $0^{\circ}$ until TLC (20\% EtOAc/hexanes) indicated ca. $90 \%$ reaction completion (total $=8$ equivalents), at which point the reaction was quenched via drop-wise addition of $20 \mathrm{~mL}$ of saturated $\mathrm{NaHCO}_{3}$. The resulting mixture was stirred for $15 \mathrm{~min}$, diluted with $\mathrm{Et}_{2} \mathrm{O}$, stirred an additional $30 \mathrm{~min}$, and the layers were separated. The aqueous layer 
was extracted $\left(3 \times \mathrm{Et}_{2} \mathrm{O}\right)$, and the resulting organic layers were combined, washed (water and saturated brine solution), dried $\left(\mathrm{MgSO}_{4}\right)$, filtered, added to $\mathrm{SiO}_{2}$ and concentrated. Flash chromatography (gradient elution: 5\% EtOAc/hexanes to 10\% EtOAc/hexanes; 2nd column: $100 \% \mathrm{CH}_{2} \mathrm{Cl}_{2}$; then $20 \%$ EtOAc/hexanes) provided $\mathbf{N} 10$ (85 mg, 79\%, 10:1 dr) as a white foam and starting ether $\mathbf{N 7}$ (8\% starting material): $\mathrm{NMR}\left(500 \mathrm{MHz}, \mathrm{CDCl}_{3}\right.$, major diastereomer) 07.25 $(\mathrm{d}, \mathrm{J}=8.5 \mathrm{~Hz}, 2 \mathrm{H}), 6.85(\mathrm{~d}, \mathrm{j}=8.7 \mathrm{~Hz}, 2 \mathrm{H}), 6.60$ (ddd, J = 16.8, 10.6, 10.6, 1H), $6.03($ app t, J = $10.9 \mathrm{~Hz}, 1 \mathrm{H}), 5.57(\operatorname{app~t}, \mathrm{J}=10.5 \mathrm{~Hz}, 1 \mathrm{H}), 5.21(\mathrm{~m}, 1 \mathrm{H}), 5.20(\mathrm{~d}, \mathrm{~J}=16.9 \mathrm{~Hz}, 1 \mathrm{H}), 5.19(\mathrm{~m}, 1 \mathrm{H})$, $5.10(\mathrm{~d}, \mathrm{~J}=10.2 \mathrm{~Hz}, 1 \mathrm{H}), 4.50(\mathrm{ABq}, \mathrm{J}=10.7$, $\mathrm{Dn}=52.4 \mathrm{~Hz}, 2 \mathrm{H}), 3.80(\mathrm{~s}, 3 \mathrm{H}), 3.68(\mathrm{ddd}, \mathrm{J}=$ 10.3, 4.7, $4.7 \mathrm{~Hz}, 1 \mathrm{H}$ ), 3.54 (ddd, J = 11.2, $5.8,5.8 \mathrm{~Hz}, 1 \mathrm{H}$ ), 3.49 (app t, J = 4.4 Hz, 1H), 3.45 (dd, $\mathrm{J}=6.9,3.7 \mathrm{~Hz}, 1 \mathrm{H}), 3.23(\mathrm{dd}, \mathrm{J}=6.8,4.2 \mathrm{~Hz}, 1 \mathrm{H}), 2.99($ app ddq, $\mathrm{J}=6.8,7.3,6.8 \mathrm{~Hz}, 1 \mathrm{H}), 2.62$ (m, 1H), $2.34(\operatorname{app~t,~J~=~5.6~Hz,~1H),~} 1.92(\mathrm{~m}, 2 \mathrm{H}), 1.83(\mathrm{~m}, 2 \mathrm{H}), 1.60(\mathrm{~m}, 1 \mathrm{H}), 1.09(\mathrm{~d}, \mathrm{~J}=6.8$ $\mathrm{Hz}, 3 \mathrm{H}), 1.00$ (d, J = 7.1 Hz, 3H), 0.99 (d, J = 6.9 Hz, 3H), $0.95(\mathrm{~m}, 3 \mathrm{H}), 0.95(\mathrm{~s}, 9 \mathrm{H}), 0.92(\mathrm{~s}, 9 \mathrm{H})$, $0.80(\mathrm{~d}, \mathrm{~J}=6.7 \mathrm{~Hz}, 3 \mathrm{H}), 0.11(\mathrm{~s}, 3 \mathrm{H}), 0.10(\mathrm{~s}, 3 \mathrm{H}), 0.09(\mathrm{~s}, 3 \mathrm{H}), 0.08(\mathrm{~s}, 3 \mathrm{H}) ;{ }^{13} \mathrm{C} \mathrm{NMR}(125 \mathrm{MHz}$, $\mathrm{CDCl}_{3}$, major diastereomer) $\mathrm{T} 159.1,134.7,134.0,132.4,131.3,129.2,129.0,128.5,117.4$, 113.6, 84.5, 81.3, 76.4, 74.9, 65.3, 55.3, 40.2, 38.4, 38.2, 36.8, 35.5, 32.1, 26.3, 26.2, 18.7, 18.6, $18.4,17.5,15.8,15.0,10.8,-3.3,-3.4,-3.6,-3.8$.

Phosphonium Salt (+)-N9: A solution of iodine (138 mg, $0.545 \mathrm{mmol})$ in $1.0 \mathrm{~mL}$ of $\mathrm{Et}_{2} \mathrm{O}$ was added drop-wise to a vigorously stirred solution of alcohol N10 (38 mg, 0.054 mmol; 10:1 mix of cis/trans diene isomers), $\mathrm{PPh}_{3}(142 \mathrm{mg}, 0.54 \mathrm{mmol}$ ) and imidazole (44 mg, $0.648 \mathrm{mmol})$ in benzene/ether $(1: 1,1.4 \mathrm{~mL})$ at $0{ }^{\circ} \mathrm{C}$. The resultant canary yellow suspension was stirred $30 \mathrm{~min}$ at $0{ }^{\circ} \mathrm{C}$ and poured into $1: 1$ water/hexanes. The layers were separated, and the aqueous layer was extracted with hexanes. The combined organic layers were washed with saturated aqueous $\mathrm{Na}_{2} \mathrm{~S}_{2} \mathrm{O}_{3}(2 \mathrm{x})$, water and brine. The clear, colorless organic layer was dried over $\mathrm{MgSO}_{4}$, filtered and concentrated. The resulting white slurry was loaded onto a plug of $\mathrm{SiO}_{2}$ with a minimal amount of $\mathrm{CH}_{2} \mathrm{Cl}_{2}$ and rapidly eluted off the column $\left(0.05 \% \mathrm{Et}_{3} \mathrm{~N} / 2 \% \mathrm{Et}_{2} \mathrm{O} /\right.$ hexanes) to afford iodide N11 as colorless oil (10:1 mixture of diene isomers; contaminated with ca. $20 \% \mathrm{PPh}_{3}$ ) 
which was taken on to the next step without further purification. ${ }^{1} \mathrm{H} \mathrm{NMR}\left(500 \mathrm{MHz}, \mathrm{C}_{6} \mathrm{D}_{6}\right.$, major diastereomer) $\mathrm{C} 7.30(\mathrm{dd}, J=8.4,1.3 \mathrm{~Hz}, 2 \mathrm{H}), 6.83(\mathrm{dd}, J=8.5,1.5 \mathrm{~Hz}, 2 \mathrm{H}), 6.72(\mathrm{ddd}, J=16.8$, 10.5, 10.5 Hz, 1H), $6.09(\operatorname{app~t}, J=10.9 \mathrm{~Hz}, 1 \mathrm{H}), 5.70(\operatorname{app~t}, J=10.5 \mathrm{~Hz}, 1 \mathrm{H}), 5.33(\mathrm{~m}, 2 \mathrm{H}), 5.18$ $(\mathrm{d}, J=16.8 \mathrm{~Hz}, 1 \mathrm{H}), 5.08(\mathrm{~d}, J=10.2 \mathrm{~Hz}, 1 \mathrm{H}), 4.55(\mathrm{ABq}, J=10.7, \square \square=51 \mathrm{~Hz}, 2 \mathrm{H}), 3.67(\mathrm{~m}$, $1 \mathrm{H}), 3.46(\mathrm{~m}, 1 \mathrm{H}), 3.35(\mathrm{~m}, 1 \mathrm{H}), 3.34(\mathrm{~s}, 3 \mathrm{H}), 3.10(\mathrm{~m}, 1 \mathrm{H}), 3.05(\mathrm{ddd}, J=9.5,9.5,1.3 \mathrm{~Hz}, 1 \mathrm{H})$, $2.72(\mathrm{~m}, 1 \mathrm{H}), 2.13(\mathrm{~m}, 2 \mathrm{H}), 2.00(\mathrm{ddd}, J=12.0,12.0,6.7 \mathrm{~Hz}, 1 \mathrm{H}), 1.88(\mathrm{~m}, 2 \mathrm{H}), 1.77(\mathrm{~m}, 1 \mathrm{H})$, $1.22(\mathrm{~d}, J=6.8 \mathrm{~Hz}, 3 \mathrm{H}), 1.11(\mathrm{~d}, J=6.8 \mathrm{~Hz}, 3 \mathrm{H}), 1.06(\mathrm{~s}, 9 \mathrm{H}), 1.03(\mathrm{~d}, J=6.8 \mathrm{~Hz}, 3 \mathrm{H}), 0.98$ (d, $J$ $=6.7 \mathrm{~Hz}, 3 \mathrm{H}), 0.96(\mathrm{~s}, 9 \mathrm{H}), 0.85(\mathrm{~d}, J=6.7 \mathrm{~Hz}, 3 \mathrm{H}), 0.17(\mathrm{~s}, 3 \mathrm{H}), 0.16(\mathrm{~s}, 3 \mathrm{H}), 0.10(\mathrm{~s}, 3 \mathrm{H}), 0.09$ $(\mathrm{s}, 3 \mathrm{H})$.

To an intimate mixture of crude iodide $\mathbf{N 1 1}(0.054 \mathrm{mmol})$ and triphenylphosphine $(28 \mathrm{mg}$, $0.108 \mathrm{mmol})$ was added diisopropylethylamine $(100 \mu \mathrm{L})$. The resulting mixture was heated $\left(95^{\circ}\right.$ C) for $8 \mathrm{~h}$, cooled and diluted with $\mathrm{CH}_{2} \mathrm{Cl}_{2}$. Flash chromatography (gradient elution, $\mathrm{CH}_{2} \mathrm{Cl}_{2} \mathrm{Z}$ $20 \% \mathrm{MeCN} / \mathrm{CH}_{2} \mathrm{Cl}_{2}$ ) furnished (+)-N9 as a white solid [55 mg, 95\% yield from alcohol $\mathrm{N10}$ ]: $[\square]_{\mathrm{D}}^{23}+18\left(c\right.$ 1.0, $\left.\mathrm{CHCl}_{3}\right) ; \mathrm{IR}\left(\mathrm{CHCl}_{3}\right) 2928,1513,1438,1249,1070,837 \mathrm{~cm}^{-1} ;{ }^{1} \mathrm{H}$ NMR $(500$ $\mathrm{MHz}, \mathrm{CDCl}_{3}$, major diastereomer) $\mathrm{T} 7.75(\mathrm{~m}, 15 \mathrm{H}), 7.23(\mathrm{~d}, J=8.3 \mathrm{~Hz}, 2 \mathrm{H}), 6.82(\mathrm{~d}, J=8.6 \mathrm{~Hz}$, 2H), 6.53 (ddd, $J=16.8,10.6,10.6 \mathrm{~Hz}, 1 \mathrm{H}), 5.90($ app t, $J=11.0 \mathrm{~Hz}, 1 \mathrm{H}), 5.52($ app t, $J=10.8$ $\mathrm{Hz}, 1 \mathrm{H}), 5.50(\operatorname{appt} \mathrm{t} J=10.7 \mathrm{~Hz}, 1 \mathrm{H}), 5.16(\mathrm{dd}, J=9.8,5.1 \mathrm{~Hz}, 1 \mathrm{H}), 5.10(\mathrm{~d}, J=16.4 \mathrm{~Hz}, 1 \mathrm{H})$, $5.05(\mathrm{~d}, J=10.1 \mathrm{~Hz}, 1 \mathrm{H}), 4.24(\mathrm{ABq}, J=10.5, \square \square=56 \mathrm{~Hz}, 2 \mathrm{H}), 3.75(\mathrm{~s}, 3 \mathrm{H}), 3.74(\mathrm{~m}, 2 \mathrm{H}), 3.43$ (m, 1H), $3.20(\mathrm{~m}, 2 \mathrm{H}), 2.94(\mathrm{app} d d q, J=6.7,6.8,6.7 \mathrm{~Hz}, 1 \mathrm{H}) 2.49(\mathrm{~m}, 1 \mathrm{H}), 2.01(\mathrm{~m}, 1 \mathrm{H}), 1.80(\mathrm{~m}$, 2H), $1.72(\mathrm{~m}, 1 \mathrm{H}), 1.55(\mathrm{~m}, 1 \mathrm{H}), 1.07(\mathrm{~d}, J=6.8 \mathrm{~Hz}, 3 \mathrm{H}), 0.92(\mathrm{~d}, J=6.9 \mathrm{~Hz}, 3 \mathrm{H}), 0.88(\mathrm{~s}, 9 \mathrm{H})$, $0.86(\mathrm{~d}, J=7.2 \mathrm{~Hz}, 3 \mathrm{H}), 0.82(\mathrm{~s}, 9 \mathrm{H}), 0.73(\mathrm{~d}, J=6.7 \mathrm{~Hz}, 3 \mathrm{H}), 0.69$ (d, $J=6.8 \mathrm{~Hz}, 3 \mathrm{H}), 0.07$ (s, $3 \mathrm{H}), 0.06(\mathrm{~s}, 3 \mathrm{H}), 0.01(\mathrm{~s}, 3 \mathrm{H}),-0.08(\mathrm{~s}, 3 \mathrm{H}) ;{ }^{13} \mathrm{C} \mathrm{NMR}\left(125 \mathrm{MHz}, \mathrm{CDCl}_{3}\right.$, major diastereomer) $\mathrm{C}$ $159.1,135.3,135.2,134.5,134.0,13.7,133.6,132.2,131.2,130.8,130.7,129.2,129.1,129.0$ $128.2,118.8,118.1,117.6,113.8,84.5,79.6,79.5,76.4,75.1,40.0,38.2,35.8,35.4,34.2,34.1$, 31.7, 26.3, 26.1, 26.0, 25.6, 18.8, 18.6, 18.3, 16.8, 15.5, 10.9, -3.2, -3.3, -3.4, -3.8; high resolution mass spectrum (ES+) m/z 947.5959 [(M-I)+; calcd for $\left.\mathrm{C}_{59} \mathrm{H}_{88} \mathrm{O}_{4} \mathrm{PSi}_{2}: 947.5950\right]$. 


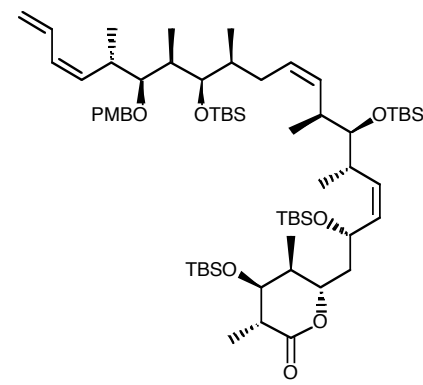

(+)-N12

Tetraene (+)-N12: Phosphonium salt (+)-N9 (80 mg, 0.074 mmol; 10:1 ratio of diene isomers), was azeotropically dried with benzene $(3 \times 1.5 \mathrm{~mL})$ using a double manifold and further dried by heating to $50{ }^{\circ} \mathrm{C}$ under vacuum ( 0.2 torr) for $12 \mathrm{~h}$. The salt was back-filled with argon, dissolved in $540 \mu \mathrm{L}$ of freshly distilled THF, sparged with argon for $15 \mathrm{~min}$, and cooled to $-20{ }^{\circ} \mathrm{C}$. The resultant solution was treated with sodium bis(trimethylsilyl)amide (1.0 M in THF, $70 \mu \mathrm{L}$ ), stirred $15 \mathrm{~min}$, warmed to $0^{\circ} \mathrm{C}$, stirred $30 \mathrm{~min}$, and re-cooled to $-24^{\circ} \mathrm{C}$. To this orange/red solution was transferred via cannula a degassed solution of aldehyde (-)-C $\mathrm{C}^{3}(29 \mathrm{mg}, 0.066 \mathrm{mmol})$ in THF ( 0.5 $\mathrm{mL}+1 \times 0.2 \mathrm{~mL}$ rinse) over $7 \mathrm{~min}$. The orange solution was allowed to slowly warm to $-8{ }^{\circ} \mathrm{C}$ over $3.25 \mathrm{~h}$. The resulting light yellow solution was quenched with saturated $\mathrm{NH}_{4} \mathrm{Cl}$ and diluted $\left(\mathrm{Et}_{2} \mathrm{O} / \mathrm{H}_{2} \mathrm{O}\right)$. The layers were separated, and the aqueous layer was extracted $\left(3 \times \mathrm{Et}_{2} \mathrm{O}\right)$. The combined organic layers were dried $\left(\mathrm{Na}_{2} \mathrm{SO}_{4}\right)$, concentrated, and chromatographed (gradient elution: $2 \% \mathrm{EtOAc} /$ hexanes to $50 \% \mathrm{EtOAc} /$ hexanes; then $40 \% \mathrm{CH}_{3} \mathrm{CN} / \mathrm{CH}_{2} \mathrm{Cl}_{2}$ ) to afford cis isomer (+)-N12 (27 mg, 36\%, 10:1 ratio of diene isomers) and phosphonium salt (+)-N9 (28 mg, 35\%; 10:1 ratio of diene isomers); [ []$_{\mathrm{D}}^{23}+31\left(c\right.$ 1.0, $\left.\mathrm{CHCl}_{3}\right)$; IR $\left(\mathrm{CHCl}_{3}\right) 2928,2856,1738,1462$, 1250, 1044, 836, $775 \mathrm{~cm}^{-1} ;{ }^{1} \mathrm{H}$ NMR $\left(500 \mathrm{MHz}, \mathrm{CDCl}_{3}\right.$, major diastereomer) $7.24(\mathrm{~d}, \mathrm{~J}=8.6$ $\mathrm{Hz}, 2 \mathrm{H}), 6.85(\mathrm{~d}, J=8.6 \mathrm{~Hz}, 2 \mathrm{H}), 6.58$ (ddd, $J=16.8,10.6,10.6 \mathrm{~Hz}, 1 \mathrm{H}), 6.00($ app t, $J=11.0 \mathrm{~Hz}$, 1H), $5.56(\operatorname{appt}, J=10.5 \mathrm{~Hz}, 1 \mathrm{H}), 5.30(\mathrm{dd}, J=11.0,8.0 \mathrm{~Hz}, 1 \mathrm{H}), 5.29(\mathrm{~m}, 1 \mathrm{H}), 5.24(\mathrm{~d}, J=10.4$ $\mathrm{Hz}, 1 \mathrm{H}), 5.19(\mathrm{dd}, J=16.8,1.5 \mathrm{~Hz}, 1 \mathrm{H}), 5.10(\mathrm{~d}, J=10.3 \mathrm{~Hz}, 1 \mathrm{H}), 4.79(\operatorname{app~t}, J=9.2 \mathrm{~Hz}, 1 \mathrm{H})$, $4.51(\operatorname{app~t}, J=10.2 \mathrm{~Hz}, 1 \mathrm{H}), 4.50(\mathrm{ABq}, J=10.6, \square \square=45 \mathrm{~Hz}, 2 \mathrm{H}), 3.79(\mathrm{~s}, 3 \mathrm{H}), 3.62(\operatorname{app~t}, J=$

\footnotetext{
${ }^{3}$ See structure C, Smith, A. B., III; Beauchamp, T. J.; LaMarche, M. J.; Kaufman, M. D.; Qiu, Y.; Arimoto, H.; Jones, D. R.; Kobayashi, K. J. Am. Chem. Soc. 2000, 122 (36), 8654-8664.
} 
$2.5 \mathrm{~Hz}, 1 \mathrm{H}), 3.48(\operatorname{app~t}, J=4.4 \mathrm{~Hz}, 1 \mathrm{H}), 3.28(\operatorname{app~t}, J=5.2 \mathrm{~Hz}, 1 \mathrm{H}), 3.23(\mathrm{dd}, J=7.1,4.0 \mathrm{~Hz}$, 1H), $2.98(a p p d d q, J=6.8,7.1,6.8 \mathrm{~Hz}, 1 \mathrm{H}), 2.62(\mathrm{~m}, 2 \mathrm{H}), 2.52(\mathrm{~m}, 1 \mathrm{H}), 1.83(\mathrm{~m}, 6 \mathrm{H}), 1.58(\mathrm{~m}$, 1H), 1.57 (dd, $J=11.2,2.2 \mathrm{~Hz}, 1 \mathrm{H}), 1.21(\mathrm{~d}, J=7.6 \mathrm{~Hz}, 3 \mathrm{H}), 1.08(\mathrm{~d}, J=6.8 \mathrm{~Hz}, 3 \mathrm{H}), 0.98(\mathrm{~d}, J=$ $6.9 \mathrm{~Hz}, 3 \mathrm{H}), 0.96(\mathrm{~d}, J=6.6 \mathrm{~Hz}, 3 \mathrm{H}), 0.95(\mathrm{~d}, J=6.8 \mathrm{~Hz}, 3 \mathrm{H}), 0.94(\mathrm{~s}, 9 \mathrm{H}), 0.90(\mathrm{~s}, 9 \mathrm{H}), 0.88(\mathrm{~d}, J$ $=6.1 \mathrm{~Hz}, 3 \mathrm{H}), 0.87(\mathrm{~s}, 9 \mathrm{H}), 0.86(\mathrm{~s}, 9 \mathrm{H}), 0.79(\mathrm{~d}, J=6.7 \mathrm{~Hz}, 3 \mathrm{H}), 0.09(\mathrm{~s}, 3 \mathrm{H}), 0.08(\mathrm{~s}, 3 \mathrm{H}), 0.07$ (s, 3H), $0.06(\mathrm{~s}, 6 \mathrm{H}), 0.05$ (s, 3H), $0.04(\mathrm{~s}, 3 \mathrm{H}), 0.01$ (s, 3H); ${ }^{13} \mathrm{C} \mathrm{NMR}\left(125 \mathrm{MHz}, \mathrm{CDCl}_{3}\right.$, major diastereomer) 7 173.2, 159.1, 135.2, 134.5, 133.6, 132.4, 132.3, 131.2, 129.1, 127.1, 117.5, $113.7,84.5,80.1,77.0,76.5,75.1,75.0,64.6,55.3,44.1,42.9,40.2,38.3,37.6,35.5,35.0,34.2$, $31.9,26.3,26.2,26.0,25.7,18.7,18.6,18.4,18.1,18.0,17.1,16.4,16.2,15.2,14.1,10.7,-3.1$, 3.3, -3.4, -4.2, -4.3, -4.5, -4.8 (2); high resolution mass spectrum $\left(\mathrm{ES}^{+}\right) \mathrm{m} / \mathrm{z} 1113.7826\left[(\mathrm{MH})^{+}\right.$; calcd for $\left.\mathrm{C}_{63} \mathrm{H}_{117} \mathrm{O}_{8} \mathrm{Si}_{4} \mathrm{Na}: 1113.7916\right]$.

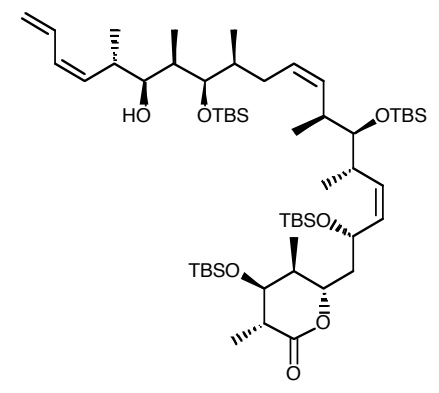

$(+)-5$

Alcohol (+)-5: At $0{ }^{\circ} \mathrm{C}$, a solution of PMB ether $(+)-\mathrm{N} 12(24 \mathrm{mg}, 0.0211 \mathrm{mmol}, 10: 1 \mathrm{mixture}$ of cis/trans diene isomers) in $\mathrm{CH}_{2} \mathrm{Cl}_{2}(2 \mathrm{~mL})$ was treated with $\mathrm{H}_{2} \mathrm{O}(100 \mu \mathrm{L})$ and $\mathrm{DDQ}(72 \mathrm{mg}$, $0.0313 \mathrm{mmol}$ ). The mixture was stirred for $10 \mathrm{~min}$ at $0{ }^{\circ} \mathrm{C}$, warmed to $\mathrm{rt}$ and stirred an additional 5 min. The mixture was quenched with saturated $\mathrm{NaHCO}_{3}$, diluted with $\mathrm{CH}_{2} \mathrm{Cl}_{2}$, and washed with $\mathrm{H}_{2} \mathrm{O}$ and saturated brine solution. The combined organic layers were dried $\left(\mathrm{MgSO}_{4}\right)$, filtered and concentrated. Flash chromatography (gradient elution; 5\% EtOAc to 10\% EtOAc/hexanes) furnished

(+)-5 (21 mg, 95\%); [ []$\left._{D}^{23}+44\left(c 1.0 \mathrm{CH}_{2} \mathrm{Cl}_{2}\right)\right] \mathrm{IR}$ (film, NaCl) 2928, 2856, 2356, 1731, 1462, 1253, 1047, 836, $775 \mathrm{~cm}^{-1} ;{ }^{1} \mathrm{H}$ NMR $\left(500 \mathrm{MHz} \mathrm{CDCl}_{3}\right) \square 6.54$ (ddd, $\left.J=16.8,10.6,10.6 \mathrm{~Hz}, 1 \mathrm{H}\right)$, 
$6.06(\operatorname{app~t}, J=10.9 \mathrm{~Hz}, 1 \mathrm{H}), 5.16(\mathrm{~m}, 6 \mathrm{H}), 4.72(\operatorname{app~t}, J=8.7 \mathrm{~Hz}, 1 \mathrm{H}), 4.43($ app t, $J=10.2 \mathrm{~Hz}$, 1H), $3.55(\mathrm{~m}, 2 \mathrm{H}), 3.24(\mathrm{~m}, 2 \mathrm{H}), 2.71(\mathrm{~m}, 1 \mathrm{H}), 2.52(\mathrm{~m} \mathrm{3H}), 1.91(\mathrm{~m}, 2 \mathrm{H}), 1.69(\mathrm{~m}, 4 \mathrm{H}), 1.51$ (ddd, $J=13.3,11.1,1.9 \mathrm{~Hz}, 1 \mathrm{H}), 1.47(\mathrm{~d}, J=2.8 \mathrm{~Hz}, 1 \mathrm{H}), 1.13(\mathrm{~d}, J=7.6 \mathrm{~Hz}, 3 \mathrm{H}), 0.88(\mathrm{~m}, 12 \mathrm{H}), 0.83$ (s, 9H), $0.83(\mathrm{~d}$, obscured, 3H), $0.82(\mathrm{~s}, 9 \mathrm{H}), 0.81(\mathrm{~s}, 9 \mathrm{H}), 0.78(\mathrm{~s}, 9 \mathrm{H}), 0.73(\mathrm{~d}, J=6.8 \mathrm{~Hz}, 3 \mathrm{H})$, 0.08 (s, 3H), $0.076(\mathrm{~s}, 3 \mathrm{H}), 0.070(\mathrm{~s}, 3 \mathrm{H}), 0.066(\mathrm{~s}, 3 \mathrm{H}), 0.058(\mathrm{~s}, 3 \mathrm{H}), 0.053(\mathrm{~s}, 3 \mathrm{H}), 0.043(\mathrm{~s}$, 3H), $0.02(\mathrm{~s}, 3 \mathrm{H}) ;{ }^{13} \mathrm{C}$ NMR $\left(125 \mathrm{MHz}, \mathrm{CDCl}_{3}\right) \mathrm{C} 173.2,135.4,134.8,133.6,132.4,132.1,131.1$, $127.3,118.4,80.1,78.3,77.1,76.1,74.9,64.7,44.1,42.9,38.0,37.5,37.4,36.4,35.1,34.2$, $32.4,26.3$ (2), 25.9, 25.7, 18.5, 18.4, 18.1, 18.0, 17.3, 17.1, 16.4, 16.0, 14.1, 13.8, 9.4, -3.1, -3.3, $-3.6,-4.2,-4.3,-4.5,-4.8(2)$; high resolution mass spectrum $\left(\mathrm{ES}^{+}\right) \mathrm{m} / \mathrm{z} 1015.7070\left[(\mathrm{M}+\mathrm{Na})^{+}\right.$; calcd for $\mathrm{C}_{55} \mathrm{H}_{108} \mathrm{O}_{7} \mathrm{Si}_{4} \mathrm{Na:}$ 1015.7090].

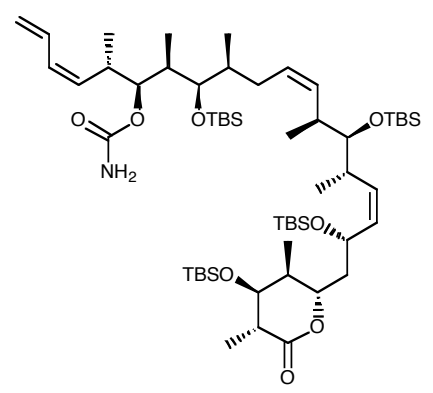

$(+)-\mathrm{N} 13$

Carbamate (+)-N13. A solution of alcohol (+)-5 (48 mg, $0.0479 \mathrm{mmol})$ in $\mathrm{CH}_{2} \mathrm{Cl}_{2}(1.6 \mathrm{~mL})$ was treated with a solution $(1 \mathrm{M}, \mathrm{PhH}) \mathrm{Cl}_{3} \mathrm{CCON}=\mathrm{C}=\mathrm{O}(144 \mathrm{~L}, 0.144 \mathrm{mmol})$ at room temperature for 30 min. Solution was loaded directly onto neutral $\mathrm{Al}_{2} \mathrm{O}_{3}$. After $4 \mathrm{~h}$, the material was flushed from the $\mathrm{Al}_{2} \mathrm{O}_{3}$ (EtOAc), concentrated, and purified by flash chromatography $(10 \%$ ethyl acetate/hexanes) providing $50 \mathrm{mg}(+)-\mathrm{N} 13(99 \%) ; \quad[\square]]_{\mathrm{D}}^{23}+31\left(c 1.0, \mathrm{CH}_{2} \mathrm{Cl}_{2}\right) ; \mathrm{IR}$ (film, $\mathrm{NaCl}$ ) 3362, 2929, 2856, 1732, 1595, 1462, 1360, 1253, 1098, 836, $775 \mathrm{~cm}^{-1} ;{ }^{1} \mathrm{H}$ NMR $(500 \mathrm{MHz}$, $\left.\mathrm{CDCl}_{3}\right) \square 6.59$ (ddd, $\left.J=16.8,10.6,10.6 \mathrm{~Hz}, 1 \mathrm{H}\right), 6.02(\operatorname{app~t}, J=11.0 \mathrm{~Hz}, 1 \mathrm{H}), 5.37(\operatorname{app~t}, J=$ $10.4 \mathrm{~Hz}, 1 \mathrm{H}), 5.30(\mathrm{~m}, 2 \mathrm{H}), 5.20(\mathrm{~d}, J=16.7 \mathrm{~Hz}, 1 \mathrm{H}), 5.13(\mathrm{~m}, 1 \mathrm{H}), 5.12(\mathrm{~d}, J=10.4 \mathrm{~Hz}, 1 \mathrm{H})$, $4.80(\operatorname{app~t}, J=8.9 \mathrm{~Hz}, 1 \mathrm{H}), 4.71(\operatorname{app~t}, J=6.0 \mathrm{~Hz}, 1 \mathrm{H}), 4.50(\operatorname{app~t}, J=10.6 \mathrm{~Hz}, 1 \mathrm{H}), 4.46$ (br s, 2H), $3.64(\operatorname{app~t}, J=2.3 \mathrm{~Hz}, 1 \mathrm{H}), 3.46(\operatorname{app~t}, J=4.5 \mathrm{~Hz}, 1 \mathrm{H}), 3.29(\operatorname{app~t}, J=5.1 \mathrm{~Hz}, 1 \mathrm{H}), 2.98$ 
(app ddq, $J=6.7,10.0,6.7 \mathrm{~Hz}, 1 \mathrm{H}$ ), $2.62(\mathrm{~m}, 2 \mathrm{H}), 2.54$ (app ddq, $J=6.6,8.2,6.6 \mathrm{~Hz}, 1 \mathrm{H}$ ), 1.90 (m, 3H), $1.80(\mathrm{ddd}, J=9.6,6.7,2.4 \mathrm{~Hz}, 1 \mathrm{H}), 1.73(\mathrm{~m}, 1 \mathrm{H}), 1.67(\mathrm{~m}, 1 \mathrm{H}), 1.57(\mathrm{~m}, 1 \mathrm{H}), 1.24(\mathrm{~m}$, 1H), $1.22(\mathrm{~d}, J=7.6 \mathrm{~Hz}, 3 \mathrm{H}), 0.99(\mathrm{~d}, J=6.8 \mathrm{~Hz}, 3 \mathrm{H}), 0.96(\mathrm{~d}, J=6.7 \mathrm{~Hz}, 6 \mathrm{H}), 0.93(\mathrm{~d}, J=6.4$ $\mathrm{Hz}, 3 \mathrm{H}), 0.92(\mathrm{~s}, 9 \mathrm{H}), 0.89(\mathrm{~s}, 9 \mathrm{H}), 0.88(\mathrm{~d}, J=5.2 \mathrm{~Hz}, 3 \mathrm{H}), 0.87(\mathrm{~s}, 9 \mathrm{H}), 0.86(\mathrm{~s}, 9 \mathrm{H}), 0.80$ (d, $J=$ $6.7 \mathrm{~Hz}, 3 \mathrm{H}), 0.10(\mathrm{~s}, 3 \mathrm{H}), 0.08(\mathrm{~s}, 3 \mathrm{H}), 0.07(\mathrm{~s}, 6 \mathrm{H}), 0.06(\mathrm{~s}, 3 \mathrm{H}), 0.05(\mathrm{~s}, 3 \mathrm{H}), 0.04(\mathrm{~s}, 3 \mathrm{H}), 0.02$ (s, 3H); ${ }^{13} \mathrm{C}$ NMR $\left(125 \mathrm{MHz}, \mathrm{CDCl}_{3}\right) \mathrm{T}$ 173.3, 156.9, 135.2, 133.6, 133.5, 132.5, 132.1, 129.9, $127.1,117.9,80.0,78.8,77.0,76.3,74.9,64.7,44.2,42.9,38.1,38.0,37.6,35.0,34.5,34.2$, $32.0,26.3,26.2,25.9,25.7,18.6,18.4,18.1,18.0,17.6,17.2,16.4,16.1,14.3,14.1,10.3,-3.1$, 3.5, -3.6, -4.2, -4.3, -4.5, -4.8 (2); high resolution mass spectrum $\left(\mathrm{ES}^{+}\right) \mathrm{m} / \mathrm{z} 1058.7114\left[(\mathrm{M}+\mathrm{Na})^{+}\right.$; calcd for $\mathrm{C}_{56} \mathrm{H}_{109} \mathrm{NO}_{8} \mathrm{Si}_{4} \mathrm{Na:}$ 1058.7128].

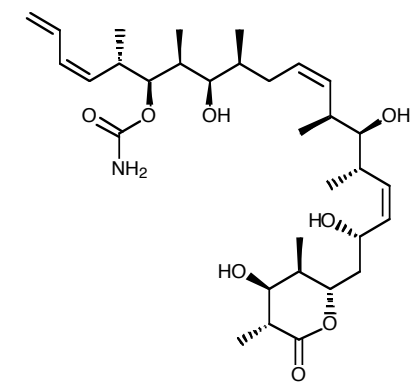

\section{(+)-14-normethyldiscodermolide}

(+)-14-normethyldiscodermolide: Carbamate (+)-N13 (11 mg, $0.0105 \mathrm{mmol})$ was dissolved in $\mathrm{MeOH}(3.7 \mathrm{~mL})$ and stirred for $15 \mathrm{~min}$ at room temperature. Aqueous hydrochloric acid (3N, 2.2 $\mathrm{mL}$ ) was added in $100 \mu \mathrm{L}$ portions over 4 hours at a rate which minimized precipitation (ca. 10 to 15 min intervals). An additional $500 \mu \mathrm{L}$ of $3 \mathrm{~N}$ aq HCl was added over $1 \mathrm{~h}$ at 15 min intervals, and the sides of the flask/stir bar were rinsed with $2 \mathrm{~mL}$ of $\mathrm{MeOH}$. After $12 \mathrm{~h}$ the solution was quenched with $\mathrm{NaHCO}_{3}$ (s), diluted with $30 \mathrm{~mL}$ of water and extracted $3 \times$ with EtOAc. The combined organic extracts were dried $\left(\mathrm{Na}_{2} \mathrm{SO}_{4}\right)$, filtered, and concentrated. Flash chromatography $\left(10 \% \mathrm{MeOH} / \mathrm{CH}_{2} \mathrm{Cl}_{2}\right)$ using washed $\mathrm{SiO}_{2}\left(10 \% \mathrm{MeOH} / \mathrm{CH}_{2} \mathrm{Cl}_{2}\right.$ then $5 \%$ $\mathrm{MeOH} / \mathrm{CH}_{2} \mathrm{Cl}_{2}$ ) furnished a white amorphous solid (+)-14-normethyldiscodermolide (5.5 mg,

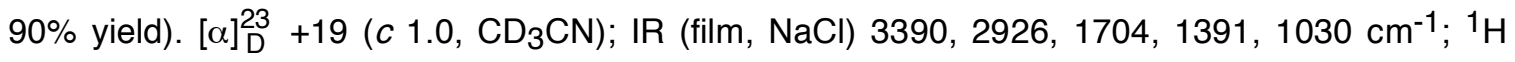


NMR $\left(500 \mathrm{MHz}, \mathrm{CD}_{3} \mathrm{CN}\right) \mathrm{G} 6.67$ (dddd, $\left.J=16.8,11.1,10.1,0.8 \mathrm{~Hz}, 1 \mathrm{H}\right), 6.05($ app t, $J=10.9 \mathrm{~Hz}$, 1H), $5.46($ app t, $J=10.4 \mathrm{~Hz}, 1 \mathrm{H}), 5.42(\operatorname{appt}, J=12.1 \mathrm{~Hz}, 1 \mathrm{H}), 5.39(\mathrm{dd}, J=11.2,8.2 \mathrm{~Hz}, 1 \mathrm{H})$, $5.25(\mathrm{~m}, 3 \mathrm{H}), 5.13(\mathrm{~d}, J=10.1 \mathrm{~Hz}, 1 \mathrm{H}), 5.05(\mathrm{br} \mathrm{s}, 2 \mathrm{H}), 4.69$ (dd, J = 7.1, $4.9 \mathrm{~Hz}, 1 \mathrm{H}), 4.52$ (dddd, $J=12.9,5.2,5.1,2.3 \mathrm{~Hz}, 1 \mathrm{H}), 4.46(\mathrm{ddd}, J=10.4,10.4,2.0 \mathrm{~Hz}, 1 \mathrm{H}), 3.64(\mathrm{dd}, J=8.5,4.5 \mathrm{~Hz}$, $1 \mathrm{H}$ ), $3.28(\mathrm{~d}, J=4.6 \mathrm{~Hz}, 1 \mathrm{H}), 3.19$ (ddd, $J=6.7,6.7,4.2 \mathrm{~Hz}, 1 \mathrm{H}), 3.14$ (ddd, $J=6.7,5.1,5.1 \mathrm{~Hz}$, 1H), $3.06(\operatorname{app~ddq,~} J=6.7,8.7,6.7 \mathrm{~Hz}, 1 \mathrm{H}), 2.81(\mathrm{~d}, J=5.2 \mathrm{~Hz}, 1 \mathrm{H}), 2.64(\mathrm{~m}, 1 \mathrm{H}), 2.63(\mathrm{~d}, J=$ $5.4 \mathrm{~Hz}, 1 \mathrm{H}), 2.58(\mathrm{~m}, 1 \mathrm{H}), 2.56(\mathrm{~d}, J=6.3 \mathrm{~Hz}, 1 \mathrm{H}), 2.42$ (app ddq, $J=9.0,6.7,6.7 \mathrm{~Hz}, 1 \mathrm{H}), 1.90$ (m, 1H), $1.86(\mathrm{~m}, 1 \mathrm{H}), 1.79$ (ddd, $J=4.2,6.9,6.9 \mathrm{~Hz}, 1 \mathrm{H}), 1.72(\mathrm{~m}, 2 \mathrm{H}), 1.61(\mathrm{~m}, 1 \mathrm{H}), 1.56$ (ddd, $J=2.5,10.7,14.1 \mathrm{~Hz}, 1 \mathrm{H}), 1.20(\mathrm{~d}, J=7.3 \mathrm{~Hz}, 3 \mathrm{H}), 1.01(\mathrm{~d}, J=6.9 \mathrm{~Hz}, 3 \mathrm{H}), 0.99(\mathrm{~d}, J=7.0 \mathrm{~Hz}$, 3H), $0.95(\mathrm{~d}, J=6.8 \mathrm{~Hz}, 3 \mathrm{H}), 0.92(\mathrm{~d}, J=6.7 \mathrm{~Hz}, 3 \mathrm{H}), 0.84(\mathrm{~d}, J=6.8 \mathrm{~Hz}, 3 \mathrm{H}), 0.83(\mathrm{~d}, J=6.6$ $\mathrm{Hz}, 3 \mathrm{H}) ;{ }^{13} \mathrm{C}$ NMR $\left(125 \mathrm{MHz}, \mathrm{CD}_{3} \mathrm{CN}\right) \mathrm{1}$ 174.5, 158.3, 135.0, 134.5, 134.0, 133.6, 133.4, 130.4, $128.3,118.5,79.3,79.1,77.9,75.5,73.1,63.8,44.0,42.3,38.5,36.8,36.6,36.3,36.2,35.0$, $32.1,19.1,18.2,16.6,15.7,15.6,13.0,9.4$; high resolution mass spectrum $\left(\mathrm{ES}^{+}\right) \mathrm{m} / \mathrm{z} 602.3651$ $\left[(\mathrm{M}+\mathrm{Na})^{+}\right.$; calcd for $\left.\mathrm{C}_{32} \mathrm{H}_{53} \mathrm{NO}_{8} \mathrm{Na:} 602.3669\right]$. 